\title{
New options for increasing the sensitivity, specificity and scope of synergistic contrast magnetic resonance imaging (scMRI) using Multiplied, Added, Subtracted and/or FiTted (MASTIR) pulse sequences
}

\author{
Ya-Jun Ma ${ }^{1}$, Hongda Shao ${ }^{1}$, Shujuan Fan $^{1}$, Xing Lu ${ }^{1}$, Jiang Du ${ }^{1}$, Ian R. Young ${ }^{2 *}$, Graeme M. Bydder ${ }^{1}$ \\ ${ }^{1}$ Department of Radiology, University of California, San Diego, CA, USA; ${ }^{2}$ Formerly Department of Electrical Engineering, Imperial College, \\ London, UK
}

Correspondence to: Graeme M. Bydder, MB, ChB. Department of Radiology, University of California, San Diego, San Diego, CA, USA. Email: gbydder@health.ucsd.edu.

\begin{abstract}
This paper reviews magnetic resonance (MR) pulse sequences in which the same or different tissue properties (TPs) such as $\mathrm{T}_{1}$ and $\mathrm{T}_{2}$ are used to contribute synergistically to lesion contrast. It also shows how synergistic contrast can be created with Multiplied, Added, Subtracted and/or fiTted Inversion Recovery (MASTIR) sequences, and be used to improve the sensitivity, specificity and scope of clinical magnetic resonance imaging (MRI) protocols. Synergistic contrast can be created from: (i) the same TP, e.g., $\mathrm{T}_{1}$ used twice or more in a pulse sequence; (ii) different TPs such as $\rho_{\mathrm{m}}, \mathrm{T}_{1}, \mathrm{~T}_{2}$, and $\mathrm{D}^{*}$ used once or more within a sequence, and (iii) additional suppression or reduction of signals from tissues and/or fluids such as fat, long $\mathrm{T}_{2}$ tissues and cerebrospinal fluid (CSF). The short inversion time (TI) inversion recovery (IR) (STIR) and double IR (DIR) sequences usually show synergistic positive contrast for lesions which have increases in both $T_{1}$ and $T_{2}$. The diffusion weighted pulsed gradient spin echo (PGSE) sequence shows synergistic contrast for lesions which have an increase in $\mathrm{T}_{2}$ and a decrease in $\mathrm{D}^{*}$; the sequence is both positively weighted for $T_{2}$ and negatively weighted for $D^{*}$. In the brain, when an IR sequence nulling white matter has subtracted from it an IR sequence nulling gray matter to form the subtracted IR (SIR) sequence, increases in the single $\mathrm{TP} \mathrm{T}_{1}$ between the two nulling points of the original two sequences generate high synergistic positive contrast. In addition, the subtraction to produce the SIR sequence reduces fat and CSF signals. To provide high sensitivity to changes in TPs in disease the SIR sequence can be used (i) alone to provide synergistic $T_{1}$ contrast as above; (ii) with $T_{2}$-weighting to provide synergistic $T_{1}$ and $T_{2}$ contrast, and (iii) with $\mathrm{T}_{2^{-}}$and $\mathrm{D}^{*}$-weighting to provide synergistic $\mathrm{T}_{1}, \mathrm{~T}_{2}$, and $\mathrm{D}^{*}$ contrast. The SIR sequence can also be used in reversed form (longer TI form minus shorter TI form) to produce very high positive synergistic $\mathrm{T}_{1}$ contrast for reductions in $\mathrm{T}_{1}$, and so increase the positive contrast enhancement produced by clinical gadolinium-based contrast agents (GBCAs) when they reduce $T_{1}$. The specificity of MRI examinations can be improved by using the reversed SIR sequence with a long echo time (TE) gradient echo as well as echo subtraction to show synergistic high contrast from $T_{1}$ and $T_{2}{ }^{*}$ shortening produced by organic iron. Other added and subtracted forms of the MASTIR sequence can be used synergistically to selectively show myelin, myelin water and fluids including blood and CSF. Protocols using MASTIR sequences to provide synergistic contrast in MRI of the brain, prostate and articular cartilage are included as illustrative examples, and the features of synergistic contrast MRI (scMRI) are compared to those of multiparametric MRI (mpMRI) and functional MRI (fMRI).
\end{abstract}

${ }^{*}$ Deceased September 27, 2019. 
Keywords: Magnetic resonance imaging (MRI); synergistic contrast MRI (scMRI); pulse sequences; Multiplied, Added, Subtracted and/or fiTted Inversion Recovery sequences (MASTIR sequences); brain; prostate; articular cartilage; contrast agents

Submitted Jun 25, 2020. Accepted for publication Jul 23, 2020. doi: $10.21037 /$ qims-20-795

View this article at: http://dx.doi.org/10.21037/qims-20-795

\section{Introduction}

In clinical magnetic resonance imaging (MRI), pulse sequences are usually described by the single tissue property (TP) such as $T_{1}$ or $T_{2}$ most responsible for the contrast they produce using terms such as $T_{1}$-weighted and $T_{2}$-weighted. With most pulse sequences, several TPs can contribute to contrast but frequently their contributions are opposed, i.e., increase in one TP (e.g., $\mathrm{T}_{1}$ ) tends to decrease lesion contrast while concurrent increase in another TP (e.g., $\mathrm{T}_{2}$ ) tends to increase lesion contrast, and the end result may be poor net, or overall contrast. To achieve useful lesion contrast with the spin echo (SE) sequence for the common change in disease of an increase in $T_{1}$ and $T_{2}$, the opposed contribution from either $T_{1}$ or $T_{2}$ is minimized. This is done by increasing the repetition time (TR) to minimize opposed $\mathrm{T}_{1}$-weighting with a heavily $\mathrm{T}_{2}$-weighted $\mathrm{SE}$ sequence, and decreasing the echo time (TE) with a heavily $\mathrm{T}_{1}$-weighted sequence to minimize opposed $\mathrm{T}_{2}$ contrast. The sequences are then described by the single TP which is the dominant source of contrast as $T_{1}$-weighted or $T_{2}$ weighted respectively.

However, there are sequences in which the contrast produced by change in two TPs, such as $T_{1}$ and $T_{2}$, is not opposed, but is synergistic so that an increase in both $T_{1}$ and $\mathrm{T}_{2}$ produces either increased positive or decreased negative contrast. This is the case for concurrent increases in $T_{1}$ and $\mathrm{T}_{2}$ with the short inversion time (TI) inversion recovery (IR) (STIR) and double IR (DIR) sequences each of which are usually both positively $T_{1}$ - and positively $T_{2}$-weighted. Simultaneous increases in $T_{1}$ and $T_{2}$ usually produce high positive lesion contrast with both sequences $(1,2)$.

Synergistic contrast may arise from:

(i) A single $\mathrm{TP}$ such as $\mathrm{T}_{1}$ which can for example contribute once to contrast during the TR period of an IR sequence and a second time in the TI period of the same sequence. $T_{1}$ can also contribute twice to contrast during both TI periods when two IR sequences with different TIs are multiplied together, or subtracted. (ii) Two or more TPs such as $\rho_{\mathrm{m}}$ (mobile proton density), $T_{1}, T_{2}, D^{*}$ (apparent diffusion coefficient), $\chi$ (susceptibility), $\delta$ (chemical shift). These may each contribute to lesion contrast during different parts of the sequence. This is the commonest form of synergistic contrast.

(iii) Additional synergistic contrast to that produced by change in one or more TPs as above. This may be produced by reducing or suppressing normal high signals of tissues and/or fluids such as those that can be produced by fat, long $\mathrm{T}_{2}$ tissues and cerebrospinal fluid (CSF).

Some of most striking images in clinical MRI result from synergistic contrast. These include STIR images of lesions that show an increase in $T_{1}$ and $T_{2}$, and diffusion weighted pulsed gradient spin echo (PGSE) images of tumors that show both an increase in $\mathrm{T}_{2}$ and a decrease in $\mathrm{D}^{*}$. It is not possible to achieve high $\mathrm{D}^{*}$-weighting with a PGSE sequence for tissues (but not moving fluids showing "pseudo-diffusion") on present day clinical scanners without it having a long $\mathrm{TE}$, and this creates $\mathrm{T}_{2}$-weighting in addition to its $\mathrm{D}^{*}$-weighting. The $\mathrm{T}_{2}$ sequence weighting is opposed to the $\mathrm{D}^{*}$ sequence weighting. As a result, clinical PGSE sequences are both positively $\mathrm{T}_{2}$-weighted and negatively $\mathrm{D}^{*}$-weighted.

Successful synergistic contrast requires a knowledge of both the sign (positive or negative) of the sequence weighting for each of the TPs contributing to the synergy, and the sign of the change in the corresponding TPs (i.e., increase or decrease, positive or negative). If the sequence weighting and TP change are both positive or both negative for each TP, their contributions to contrast are positive, but if one is positive and the other is negative the contrast is opposed, and the net result may be little or no useful contrast.

The objectives of this paper are to describe synergistic contrast, review the relevant history of lesion contrast in clinical MRI and highlight new options for creating synergistic contrast using Multiplied, Added, Subtracted 
and/or fiTted IR (MASTIR) pulse sequences.

The paper includes brief summaries of how common TPs change in disease, and the concept of pulse sequences as TP-filters which is used to describe the signal, contrast and weighting of MR images. More detail about these two topics is available in two previous papers $(3,4)$. Particular examples of MASTIR sequences are described, together with illustrative protocols for examinations of the central nervous system (CNS), prostate and articular cartilage using sequences that utilize synergistic contrast.

\section{Synergistic contrast MRI (scMRI)}

As mentioned in the Introduction, synergistic contrast can arise from:

(i) A single $\mathrm{TP}$ used twice or more in a sequence, for example, $T_{1}$ in a single IR sequence used in the $T_{1}$ dependent TR segment of the sequence as well as the TI dependent segment. It can also arise in DIR sequences when two TI segments are multiplied together, and in the subtracted IR (SIR) sequence when a TI segment with an intermediate $\mathrm{TI}_{\mathrm{i}}$ is subtracted from one with a short $\mathrm{TI}_{\mathrm{s}}$.

Synergistic contrast may arise for $\mathrm{T}_{2}$ when imaging ultrashort $\mathrm{T}_{2}$ tissues with an IR sequence using a long adiabatic inversion pulse to invert and null long $T_{2}$ signals while ultrashort $T_{2}$ tissues that are saturated by the inversion pulse recover, and following this after the $90^{\circ}$ excitation pulse by echo subtraction (ES), i.e., subtraction of a long TE image from a shorter one. The two effects firstly from the inversion pulse and nulling, and secondly from the decay in transverse magnetization $\left(M_{X}\right)$ produce synergistic negative contrast when there is an increase in $T_{2}$ in ultrashort $T_{2}$ tissues.

Another example comes from angiography where highlighted inflowing blood present in one image can have subtracted from it dephased low signal flowing blood in another registered image to produce an angiogram with synergistic high contrast for flowing blood that is greater than that seen on either of the two contributing images. The subtraction cancels out signals from stationary tissues or fluids producing further synergistic contrast.

(ii) Two or more different TPs. Clinical pulse sequences have a basic structure consisting of $\rho_{\mathrm{m}}$, $T_{1}$, and $T_{2}$ segments as seen in $\mathrm{SE}$ sequences. There are additional options which can be added such as those for $T_{1}$ dependent inversion pulses and $D^{*}$ sensitization. In most circumstances $\rho_{\mathrm{m}}$ is a minor determinant of contrast and $T_{1}, T_{2}$, and $D^{*}$ are major determinants. The most common change in TPs in disease is a concurrent increase in $\rho_{\mathrm{m}}$, $\mathrm{T}_{1}, \mathrm{~T}_{2}$. In this situation, with a conventional $\mathrm{SE}$ sequence, the contrast developed by an increase in $\mathrm{T}_{1}$ is negative while that developed by an increase in $T_{2}$ is positive, so that simultaneous increases in $\mathrm{T}_{1}$ and $\mathrm{T}_{2}$ produce opposed contrast and the net, or overall, contrast is reduced. To avoid this problem, as explained previously, $\mathrm{T}_{1}$-weighted sequences use a short $\mathrm{TE}$ to minimize the opposed $\mathrm{T}_{2}$ contrast, and $\mathrm{T}_{2}$-weighted sequences use a long $\mathrm{TR}$ to minimize the opposed $T_{1}$ contrast. The dominant source of contrast in the resulting sequences is then a single $T P$, i.e., $T_{1}$ or $T_{2}$ and the sequences are described as $T_{1}$-weighted or $T_{2}$-weighted respectively.

In particular circumstances, such as certain forms of the STIR and the DIR sequences, the $T_{1}$ contrast produced by an increase in $T_{1}$ is positive, and so is the $T_{2}$ contrast produced by an increase in $T_{2}$. The effects of the concurrent increases in $T_{1}$ and $T_{2}$ are therefore synergistic and usually result in high positive lesion contrast. The STIR and DIR sequences in these circumstances are each both positively $T_{1}$ - and positively $T_{2}$-weighted.

(iii) Additional suppression or reduction of signals from normal tissues and/or fluids such as fat, long $\mathrm{T}_{2}$ tissues and CSF. There may be little contrast between high signal lesions and high signal fat, long $\mathrm{T}_{2}$ tissues, or fluids. Reduction in the normal signal from these latter tissues or fluids (using the same or different TPs as those used to create the original contrast) can increase the contrast between the high signal lesions and the zero or low signal suppressed tissues and/or fluids. It may also result in a more appropriate dynamic range.

In a tissue with a mixture of ultrashort $T_{2}$ and long $\mathrm{T}_{2}$ tissues, for example, low abundance ultrashort $\mathrm{T}_{2}$ tissues may only become apparent if the more abundant signals from the long $\mathrm{T}_{2}$ tissues are reduced or suppressed. This also applies to edema in yellow bone marrow, where suppression of the more abundant fat signal may be necessary to show the lower concentration edema.

One or both of mechanisms (i) and (ii) described above 
may be used in any one synergistic contrast sequence with, or without, the supplementary synergistic contrast from suppression of signals from normal tissues or fluids described in (iii). Achievement of synergistic contrast requires a knowledge of the sign of sequence weighting of the TPs involved as well as the sign of the change in each TP.

There are major and minor determinants of contrast. $T_{1}$ and $T_{2}$ are normally major determinants, and $\rho_{\mathrm{m}}$ is a minor one but exceptions to this for $\rho_{\mathrm{m}}$ are: (i) magnetization transfer (MT), and (ii) $\rho_{\mathrm{m}}$ contributions to water and fat in red and yellow bone marrow as well as brown adipose tissue (BAT) where selectively saturation of fat may produce a large reduction in the overall $\rho_{\mathrm{m}}$ and high tissue or lesion contrast.

There are three situations within sequences where the ability to reverse the sign of the weighting of a segment of the sequence is of critical importance for achieving synergistic contrast. These are firstly, reversal of the sign of $T_{1}$ contrast produced by a change in $T_{1}$ with IR sequences by using different TIs together with magnitude reconstruction. Secondly, reversal of the sign of $T_{2}$ contrast produced by a change in $T_{2}$ with $\mathrm{SE}$ sequences by subtraction of a longer TE image from a shorter TE one, i.e., ES. Thirdly, reversal of the sign of diffusion contrast produced by PGSE sequences by subtraction of a high $b$ value (e.g., $500-1,500 \mathrm{~s} / \mathrm{mm}^{2}$ ) image from a low b value (e.g., $0-20 \mathrm{~s} / \mathrm{mm}^{2}$ ) image, i.e., diffusion subtraction (DS). This ability to change the sign of the sequence weighting and the resulting contrast for $T_{1}, T_{2}$ and $D^{*}$ is crucial for creating synergistic contrast from both positive and negative changes in $\mathrm{T}_{1}, \mathrm{~T}_{2}$ and $\mathrm{D}^{*}$ in disease.

In addition to reversal of the sign of the sequence weighting within the segments of a sequence as above, it is also possible to reverse the order of subtraction of sequences as a whole, and so reverse the contrast of the subtracted sequences. In the rest of this paper, reversed subtraction of this type is described by the abbreviation $r$ for reversal.

Using the same change in a TP twice or more in the same sequence may result in higher synergistic contrast than just using it once. Using changes in different TPs may also be effective in increasing overall contrast. This is because $T_{1}, T_{2}$ and $D^{*}$ often change concurrently in disease and using synergistic contrast to exploit the lesion contrast developed by each of these TPs may result in higher overall contrast. These approaches are targeted at increasing sequence sensitivity.

Synergistic contrast can also be used to improve sequence specificity by, for example, using both the reduction in $T_{1}$ and $\mathrm{T}_{2}{ }^{*}$ produced by organic iron to provide high contrast visualization of its effects.

\section{TPs: normal and abnormal}

\section{Normal TPs}

In common usage, and in the following sections, both tissues and fluids are included in the general concept of TPs unless they are separately specified. There is a great deal of information available on TPs in health and disease and only a few points and principles relevant to later sections in this paper are mentioned below.

\section{General features}

(i) The values of $\rho_{\mathrm{m}}, T_{1}$ and $T_{2}$ in different tissues and fluids show parallels. Fluids generally have longer $T_{1}$ and $T_{2} s$ than tissues. Increased water content is associated with longer values of $T_{1}$ and $T_{2}$. $D^{*}$ also generally increases with increased water content.

(ii) Differences in $\rho_{\mathrm{m}}$ in disease are usually much smaller than those in $\mathrm{T}_{1}, \mathrm{~T}_{2}$ and $\mathrm{D}^{*}$.

(iii) Some organs such as the brain and kidney have two separated normal tissues (white and gray matter; cortex and medulla). Others have two distinct tissues which are mixed together, e.g., fibroglandular tissue in the prostate.

(iv) It is possible to categorize tissues and fluids by their $\mathrm{T}_{2} \mathrm{~s}$ or $\mathrm{T}_{2}{ }^{*} \mathrm{~s}$ and this corresponds to their detectability with different pulse sequences: long $T_{2}$ fluids $(100 \mathrm{~ms}$ +) (most sequences), intermediate $T_{2}$ tissues (10-100 $\mathrm{ms})$ (most sequences), short $\mathrm{T}_{2}$ tissues $(1-10 \mathrm{~ms})$ (gradient echo and some $\mathrm{SE}$ sequences), ultrashort $\mathrm{T}_{2}$ tissues (0.1-0.01 ms) [ultrashort TE (UTE) and similar sequences] and supershort $T_{2}$ tissues $(<0.1 \mathrm{~ms})$ [indirect detection with Magnetization Transfer (MT) sequences].

The $T_{1}$ s of tissues can be classified using the same general categories. $T_{1}$ increases with field strength. $T_{2}$ decreases to a lesser degree with field strength.

\section{Specific features}

(i) Fat in the form of triglycerides has a specific chemical shift of -3.3 ppm relative to water.

(ii) Normal paramagnetics including organic iron components tend to reduce $T_{1}$ and $T_{2}{ }^{*}$.

\section{Abnormal TPs}

\section{General features}

(i) The most common abnormality in disease (e.g., 


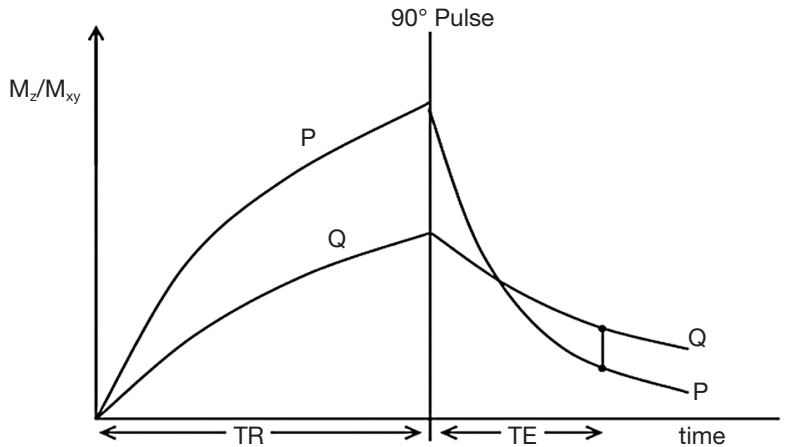

Figure 1 Composite diagram for the SE sequence. This shows recovery of the $M_{z}$ for time TR, followed by decay of the transfer magnetization $\left(M_{x y}\right)$ for time $\mathrm{TE}$ after the $90^{\circ}$ pulse, for a short $T_{1}$ short $T_{2}$ tissue $P$, and a longer $T_{1}$ longer $T_{2}$ tissue $Q$. The contrast between the two tissues is proportional to the difference between the two curves (vertical line) at time TE during the dc. SE, spin echo; TR, repetition time; TE, echo time; $M_{z}$, longitudinal magnetization; dc, data collection.

inflammation, vasogenic edema, chronic cerebral infarction, demyelination and many tumors) is an increase in $\rho_{\mathrm{m}}, \mathrm{T}_{1}, \mathrm{~T}_{2}$ and $\mathrm{D}^{*}$. Small changes in $\mathrm{T}_{1}$, $\mathrm{T}_{2}$ and $\mathrm{D}^{*}$ which are not detectable when change in only one TP is used to produce contrast, may become apparent if several different TPs are used together in the same sequence to create synergistic contrast.

(ii) $\mathrm{D}^{*}$ is decreased in acute cerebral infarction, other acute diseases and many tumors but is increased in many other conditions.

(iii) The prostate is unusual in that tumors in the peripheral and transitional zones typically show a decrease in each of $T_{1}, T_{2}$ and $D^{*}$ relative to normal fibroglandular tissue.

(iv) Abnormal paramagnetics including organic iron decrease $T_{1}, T_{2}$ and/or $T_{2}{ }^{*}$ which is the opposite to the common change in disease.

(v) Contrast agents also decrease $T_{1}, T_{2}$ and/or $T_{2}{ }^{*}$ with the emphasis on $T_{1}$ with clinical gadolinium-based contrast agents (GBCAs), and $\mathrm{T}_{2} / \mathrm{T}_{2}{ }^{*}$ as well as $\mathrm{T}_{1}$ with Magnetic Iron Oxide Particles (MIOPs).

\section{Specific features}

(i) Fat can be increased in the liver and other organs in disease.

(ii) Iron accumulates in the liver and heart generally in disease as well as in the brain focally with demyelination, and after hemorrhage and infarction as well as in gray matter.

(iii) Myelin may be reduced in demyelinating disease and other conditions affecting CNS.

\section{Pulse sequences as TP-filters $(3,4)$}

The usual explanation of image signal and contrast for a SE sequence follows firstly longitudinal magnetization $\left(M_{z}\right)$ over time, and secondly $M_{x y}$ over time after the application of a $90^{\circ}$ pulse which converts $M_{z}$ to $M_{x y}$ (Figure 1). Contrast between two tissues, such as $\mathrm{P}$ with a shorter $\mathrm{T}_{1}$ and $\mathrm{T}_{2}$, and $Q$ with a longer $T_{1}$ and $T_{2}$ is shown by the difference in $M_{x y}$ at TE in Figure 1.

After the $90^{\circ}$ pulse there is a $T_{1}$ dependent period when $T_{1}$ contrast is dominant with the $M_{x y}$ of $P$ greater than that of $\mathrm{Q}$. This is followed by a $T_{2}$ dependent period when the $M_{x y}$ of $Q$ is greater than that of $P$.

Voxel signal $\mathrm{S}$ for a SE sequence is derived from the simplified Bloch equations so that:

$$
\mathrm{S}=\mathrm{K} \rho_{\mathrm{m}}\left(1-\mathrm{e}^{-\mathrm{t} / \mathrm{T} 1}\right) \mathrm{e}^{-\mathrm{t} / \mathrm{T} 2}
$$

where $\mathrm{K}$ is a scaling function, $\rho_{\mathrm{m}}$ is the mobile proton density, and $t$ is time. This equation describes $\rho_{\mathrm{m}}$ in the first segment, recovery of $M_{z}$ over time in the second segment which is in parentheses, and decay of $M_{x y}$ over time in the third segment, with equations in the second and third segments of the forms $y=1-\mathrm{e}^{-\mathrm{x}}$ and $\mathrm{y}=\mathrm{e}^{-\mathrm{x}}$ respectively.

For the fixed times TR and TE of the SE sequence it is possible to regard the variables of interest as the TPs $\rho_{\mathrm{m}}, \mathrm{T}_{1}$ and $\mathrm{T}_{2}$ so that Eq. [1] becomes:

$$
\mathrm{S}=\mathrm{K} \rho_{\mathrm{m}}\left(1-\mathrm{e}^{-\mathrm{TR} / \mathrm{T1} 1}\right) \mathrm{e}^{-\mathrm{TE} / \mathrm{T} 2}
$$

or:

$$
\mathrm{S}=\mathrm{KS} \rho_{\mathrm{m}} \mathrm{S}_{\mathrm{T} 1} \mathrm{~S}_{\mathrm{T} 2}
$$

where the signals for the three segments $S \rho_{\mathrm{m}}, \mathrm{S}_{\mathrm{T} 1}$ and $\mathrm{S}_{\mathrm{T} 2}$ are given by:

$$
\mathrm{S} \rho_{\mathrm{m}}=\rho_{\mathrm{m}}, \quad \mathrm{S}_{\mathrm{T} 1}=1-\mathrm{e}^{-\mathrm{TR} / \mathrm{T} 1}, \quad \mathrm{~S}_{\mathrm{T} 2}=\mathrm{e}^{-\mathrm{TE} / \mathrm{T} 2}
$$

The Eqs. for the second and third segments in Eq. [4] are of the forms $y=1-e^{-1 / x}$ and $y=e^{-1 / x}$ respectively, which are quite different from the forms $\mathrm{y}=1-\mathrm{e}^{-\mathrm{x}}$ and $\mathrm{y}=\mathrm{e}^{-\mathrm{x}}$ in the second and third segments of Eq. [1]. In Eq. [4] the variable 


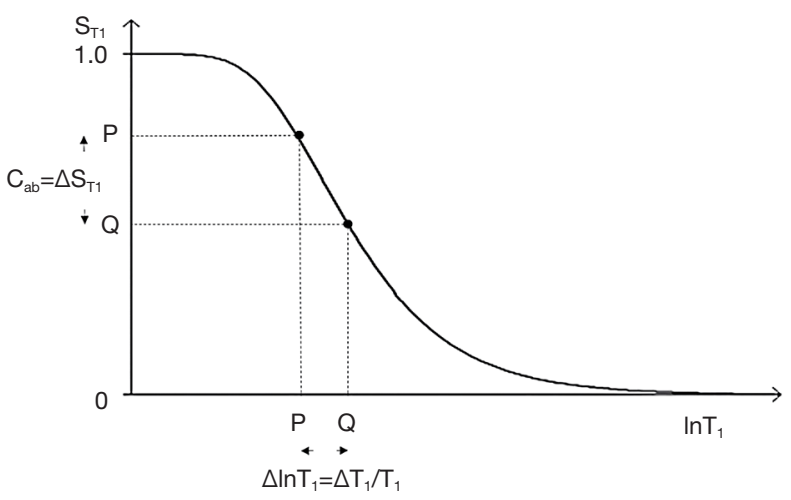

Figure 2 The $\mathrm{SE} \mathrm{T}_{1}$-filter sequence. Relationship between change in $\ln \mathrm{T}_{1}\left(=\Delta \mathrm{T}_{1} / \mathrm{T}_{1}\right)$ and change in signal (or contrast $\left.\mathrm{C}_{\mathrm{ab}}\right) \Delta \mathrm{S}_{\mathrm{T} 1}$. The positive difference $\Delta \ln \mathrm{T}_{1}=\Delta \mathrm{T}_{1} / \mathrm{T}_{1}$ from $\mathrm{P}$ to $\mathrm{Q}$ along the $\mathrm{X}$ axis produces a negative change in signal $\Delta \mathrm{S}_{\mathrm{T} 1}$ from $\mathrm{P}$ to $\mathrm{Q}$ along the $\mathrm{Y}$ axis in a region where the $\mathrm{T}_{1}$-filter is steeply sloping. This is the absolute contrast $\mathrm{C}_{\mathrm{ab}}$. The change $\Delta \ln \mathrm{T}_{1}$ would produce little or no contrast if it was opposite either the high or the low signal plateau regions of the filter. The slope of the curve between $\mathrm{P}$ and $\mathrm{Q}$ which is the sequence weighting is negative. SE, spin echo.

t shown in Eq. [1] has become a constant (either TR or $\mathrm{TE}$ ) and the two time constants $T_{1}$ and $T_{2}$ have become variables.

The three segments of Eqs. [2]-[4] have the features of a linear or exponential filter for $\rho_{\mathrm{m}}$, depending on whether the $\mathrm{X}$ axis is linear or natural logarithmic (ln), a low pass filter for $\mathrm{T}_{1}$ (Figure 2) and a high pass filter for $\mathrm{T}_{2}$ (Figure 3).

The signal levels on images are given by Eqs. [3] and [4] for $\rho_{\mathrm{m}}, \mathrm{S}_{\mathrm{T} 1}$ and $\mathrm{S}_{\mathrm{T} 2}$ and correspond to the brightness of tissues and fluids seen on images.

Absolute contrast $\left(\mathrm{C}_{\mathrm{ab}}\right)$ or difference in signal $\Delta \mathrm{S}_{\mathrm{T} 1}$ produced by a difference $\Delta \ln \mathrm{T}_{1}$ between two tissues $\mathrm{P}$ and $\mathrm{Q}$ is shown in Figure 2. A positive change from $\mathrm{P}$ to $\mathrm{Q}$ of $\Delta \ln T_{1}$ along the $\mathrm{X}$ axis produces a negative change from $\mathrm{P}$ to $\mathrm{Q}$, or negative contrast $\mathrm{C}_{\mathrm{ab}}=\Delta \mathrm{S}_{\mathrm{T} 1}$ along the $\mathrm{Y}$ axis.

The equation for $\mathrm{C}_{\mathrm{ab}}$ is:

$$
\mathrm{C}_{\mathrm{ab}}=\Delta \mathrm{S}_{\mathrm{T}_{1}}=\frac{\partial \mathrm{S}_{\mathrm{T}_{1}}}{\partial \mathrm{T}_{1}} \times \Delta \mathrm{T}_{1}
$$

where $\frac{\partial \mathrm{S}_{\mathrm{T}_{1}}}{\partial \mathrm{T}_{1}}$ is the first partial derivative or the slope of the $\mathrm{T}_{1}$-filter and $\Delta \mathrm{T}_{1}$ is the change in $\mathrm{T}_{1}$ using a linear $\mathrm{X}$ axis.

Using a $\ln \mathrm{X}$ axis and noting that $\Delta \ln \mathrm{T}_{1}=\frac{\Delta \mathrm{T}_{1}}{\mathrm{~T}_{1}}$ and that

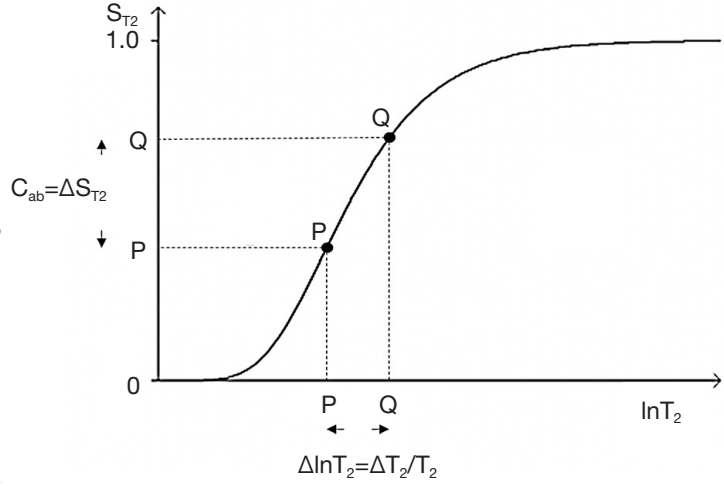

Figure 3 The $\mathrm{SE}$ sequence $\mathrm{T}_{2}$-filter. Plot of $\mathrm{S}_{\mathrm{T} 2}$ against $\ln \mathrm{T}_{2}$ for two tissues, $\mathrm{P}$ and $\mathrm{Q}$. The positive difference $\Delta \ln \mathrm{T}_{2}=\Delta \mathrm{T}_{2} / \mathrm{T}_{2}$ from $\mathrm{P}$ to $\mathrm{Q}$ along the $\mathrm{X}$ axis produces a positive difference in signal (or contrast $\mathrm{Cab}$ ) $\Delta \mathrm{S}_{\mathrm{T} 2}$ from $\mathrm{P}$ to $\mathrm{Q}$ along the $\mathrm{Y}$ axis where it corresponds with a sloping region of the $\mathrm{T}_{2}$-filter. Little or no difference in signal would be produced by $\Delta \ln T_{2}$ if it was opposite either the upper or the lower flat regions of the curve. The slope of the $\mathrm{T}_{2}$-filter between $\mathrm{P}$ and $\mathrm{Q}$ is its sequence weighting which is positive. SE, spin echo.

$$
\begin{gathered}
\frac{\mathrm{dy}}{\mathrm{d}(\ln \mathrm{x})}=\mathrm{x} \frac{\mathrm{dy}}{\mathrm{dx}}, \text { for } \mathrm{T}_{1} \text { Eq. [5] becomes: } \\
\mathrm{C}_{\mathrm{ab}}=\Delta \mathrm{S}_{\mathrm{T}_{1}}=\frac{\partial \mathrm{S}_{\mathrm{T}_{1}}}{\partial \ln \mathrm{T}_{1}} \times \frac{\Delta \mathrm{T}_{1}}{\mathrm{~T}_{1}}
\end{gathered}
$$

where $\frac{\partial \mathrm{S}_{\mathrm{T}_{1}}}{\partial \ln \mathrm{T}_{1}}$ is the slope of the filter, or the first partial derivative using a $\ln X$ axis and $\frac{\Delta T_{1}}{T_{1}}$ is the fractional change

For the $\mathrm{T}_{1}$ filter, positive change from $\mathrm{P}$ to $\mathrm{Q}$ along the $\mathrm{X}$ axis results in negative change from $\mathrm{P}$ to $\mathrm{Q}$ along the $\mathrm{Y}$ axis, i.e., negative contrast $\mathrm{C}_{\mathrm{ab}}$. The slope of the curve, which is the sequence weighting for the $T_{1}$ segment, is negative.

For the $T_{2}$ filter (Figure 3), positive change $\Delta \ln T_{2}$ from $\mathrm{P}$ to $\mathrm{Q}$ along the $\mathrm{X}$ axis results in positive change $\Delta \mathrm{S}_{\mathrm{T} 2}$ from $\mathrm{P}$ to $\mathrm{Q}$ along the $\mathrm{Y}$ axis and positive contrast $\mathrm{C}_{\mathrm{ab}}$. The slope of the filter, which is the sequence weighting for the $\mathrm{T}_{2}$ segment, is positive.

Increase in TR shifts the tail of the $T_{1}$ filter in Figure 2 to the right. Increase in TE shifts the higher part of the $\mathrm{T}_{2}$ filter in Figure 3 to the right.

Sequence weighting is maximized where $T R=T_{1}$ and $\mathrm{TE}=\mathrm{T}_{2}$ (using a $\ln \mathrm{X}$ scale) for the $\mathrm{T}_{1}$ and $\mathrm{T}_{2}$ filters respectively. 

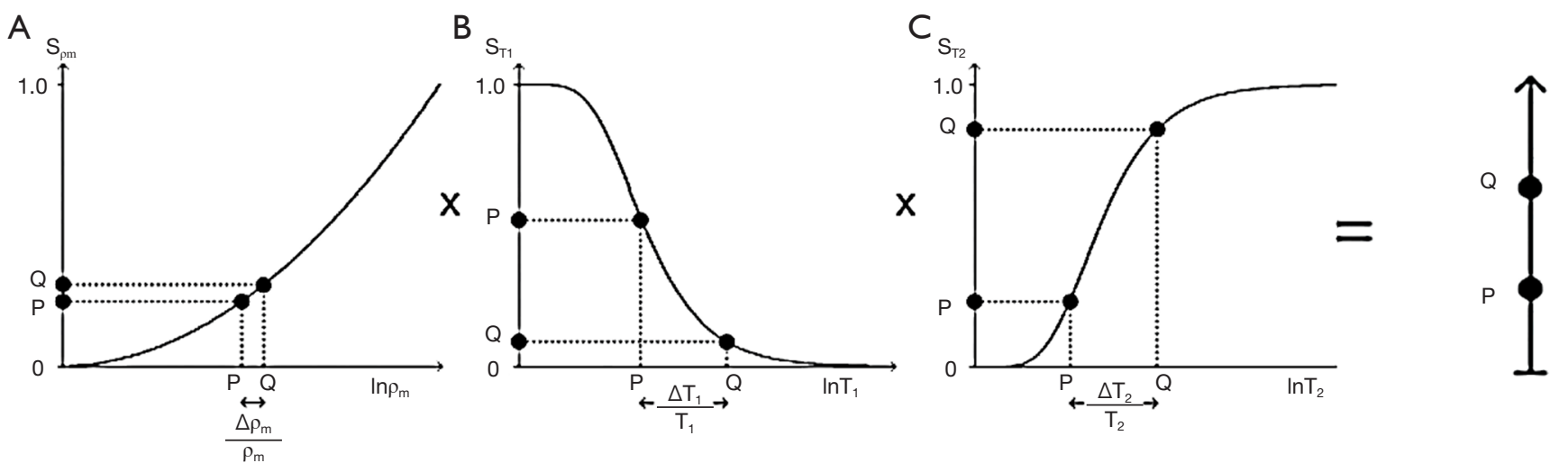

Figure 4 The SE sequence showing its $\rho_{\mathrm{m}}$-filter (A), $\mathrm{T}_{1}$-filter (B) and $\mathrm{T}_{2}$-filter $(\mathrm{C})$. Using a $\log$ TP scale the $\rho_{\mathrm{m}}$-filter is exponential, the $\mathrm{T}_{1^{-}}$ filter is a low pass sigmoid and the $\mathrm{T}_{2}$-filter is a high pass sigmoid. The signals which $\mathrm{P}$ and $\mathrm{Q}$ produce with each filter in (A), (B) and (C) are multiplied together to give the overall contrast shown at the right of the diagram. The slopes of $\rho_{\mathrm{m}}, \mathrm{T}_{1}$ and $\mathrm{T}_{2}$-filters from $\mathrm{P}$ to $\mathrm{Q}$ are their respective sequence weightings. They are positive for $\rho_{\mathrm{m}}$, negative for $T_{1}$ and positive for $T_{2}$. The contrast produced in changing positively from $\mathrm{P}$ to $\mathrm{Q}$ along the $\mathrm{X}$ axis is positive for the $\rho_{\mathrm{m}}$-filter, negative for the $\mathrm{T}_{1}$-filter and positive for the $\mathrm{T}_{2}$-filter. $\mathrm{SE}$, spin echo; TP, tissue property.

For fractional contrast $\mathrm{C}_{\mathrm{fr}}=\Delta \mathrm{S} / \mathrm{S}$ (rather than $\mathrm{C}_{\mathrm{ab}}=\Delta \mathrm{S}$ ) Eqs. [5] and [6] are divided by $S_{\mathrm{T} 1}$ and $\mathrm{S}_{\mathrm{T} 2}$ respectively for non-zero values of $S_{\mathrm{T} 1}$ and $S_{\mathrm{T} 2}$. The sequence weightings then become the normalized partial derivatives $\frac{1}{\mathrm{~S}_{\mathrm{T}_{1}}} \frac{\partial \mathrm{S}_{\mathrm{T}_{1}}}{\partial \mathrm{T}_{1}}$ and $\frac{1}{\mathrm{~S}_{\mathrm{T}_{1}}} \frac{\partial \mathrm{S}_{\mathrm{T}_{1}}}{\partial \ln \mathrm{T}_{1}}$ for linear and $\ln \mathrm{T}_{1}$ axes respectively for the $T_{1}$ filter. The same applies to the $T_{2}$ filter where the normalized partial derivatives for linear and $\ln \mathrm{X}$ axes are $\frac{1}{\mathrm{~S}_{\mathrm{T}_{1}}} \frac{\partial \mathrm{S}_{\mathrm{T}_{1}}}{\partial \ln \mathrm{T}_{1}}$ and $\frac{1}{\mathrm{~S}_{\mathrm{T}_{1}}} \frac{\partial \mathrm{S}_{\mathrm{T}_{1}}}{\partial \ln \mathrm{T}_{1}}$ respectively.

So, for $T_{1}$ :

$$
\mathrm{C}_{\mathrm{fr}}=\frac{1}{\mathrm{~S}_{\mathrm{T}_{1}}} \frac{\partial \mathrm{S}_{\mathrm{T}_{1}}}{\partial \ln \mathrm{T}_{1}} \times \frac{\Delta \mathrm{T}_{1}}{\mathrm{~T}_{1}}
$$

For $\mathrm{T}_{2}$ :

$$
\mathrm{C}_{\mathrm{fr}}=\frac{1}{\mathrm{~S}_{\mathrm{T}_{2}}} \frac{\partial \mathrm{S}_{\mathrm{T}_{2}}}{\partial \ln \mathrm{T}_{2}} \times \frac{\Delta \mathrm{T}_{2}}{\mathrm{~T}_{2}}
$$

The filters can be considered separately (i.e., the univariate model for each TP alone, as above), or be combined in the multivariate model. This shows the contributions of the sequence weightings and changes in TPs to overall contrast from each of $\rho_{\mathrm{m}}, \mathrm{T}_{1}$ and $\mathrm{T}_{2}$ and is shown for a SE sequence in Figure 4.

The contributions of the TPs to contrast, are for each TP, its sequence weighting multiplied by the change in TP. The relative contributions of each TP to sequence and image weighting can be calculated and expressed as ratios (3).

From Eq. [3] using a $\ln X$ axis:

$$
\begin{aligned}
\Delta \mathrm{S} & =\frac{\partial \mathrm{S}_{\rho_{\mathrm{m}}}}{\partial \ln \rho_{\mathrm{m}}} \mathrm{S}_{\mathrm{T}_{1}} \mathrm{~S}_{\mathrm{T}_{2}} \times \frac{\Delta \rho_{\mathrm{m}}}{\rho_{\mathrm{m}}}+\mathrm{S}_{\rho_{\mathrm{m}}} \frac{\partial \mathrm{S}_{\mathrm{T}_{1}}}{\partial \ln \mathrm{T}_{1}} \mathrm{~S}_{\mathrm{T}_{2}} \times \frac{\Delta \mathrm{T}_{1}}{\mathrm{~T}_{1}} \\
& +\mathrm{S}_{\rho_{\mathrm{m}}} \mathrm{S}_{\mathrm{T}_{1}} \frac{\partial \mathrm{S}_{\mathrm{T}_{2}}}{\partial \ln \mathrm{T}_{2}} \times \frac{\Delta \mathrm{T}_{2}}{\mathrm{~T}_{2}}
\end{aligned}
$$

Normalizing Eq. [9] by dividing it by $\mathrm{S}$ for non-zero values of $S, \rho_{\mathrm{m}}, S_{\mathrm{T} 1}$ and $S_{\mathrm{T} 2}$ gives:

$$
\begin{aligned}
\mathrm{C}_{\mathrm{fr}} & =\frac{\Delta \mathrm{S}}{\mathrm{S}}=\frac{1}{\mathrm{~S}_{\rho_{\mathrm{m}}}} \frac{\partial \mathrm{S}_{\rho_{\mathrm{m}}}}{\partial \ln \rho_{\mathrm{m}}} \times \frac{\Delta \rho_{\mathrm{m}}}{\rho_{\mathrm{m}}}+\frac{1}{\mathrm{~S}_{\mathrm{T}_{1}}} \frac{\partial \mathrm{T}_{1}}{\partial \ln \mathrm{T}_{1}} \times \frac{\Delta \mathrm{T}_{1}}{\mathrm{~T}_{1}} \\
& +\frac{1}{\mathrm{~S}_{\mathrm{T}_{2}}} \frac{\partial \mathrm{S}_{\mathrm{T}_{2}}}{\partial \ln \mathrm{T}_{2}} \times \frac{\Delta \mathrm{T}_{2}}{\mathrm{~T}_{2}}
\end{aligned}
$$

So, the overall fractional contrast $\mathrm{C}_{\mathrm{fr}}$ using a $\ln \mathrm{X}$ axis is given by:

$$
\mathrm{C}_{\mathrm{fr}}=\sum_{\mathrm{TP}} \frac{1}{\mathrm{~S}_{\mathrm{TP}}} \frac{\partial \mathrm{S}_{\mathrm{TP}}}{\partial \ln \mathrm{TP}} \times \frac{\Delta \mathrm{TP}}{\mathrm{TP}}
$$

Thus, the total fractional contrast $\mathrm{C}_{\mathrm{fr}}$ is the sum of the contributions to contrast from each TP. The contrast from each TP is the normalized first partial derivative multiplied by the fractional change in TP. If both these quantities are positive, or both are negative, a positive addition to the overall $\mathrm{C}_{\mathrm{fr}}$ results. If one quantity is negative and the other is positive a reduction in overall $\mathrm{C}_{\mathrm{fr}}$ results. To achieve synergistic contrast, contributions of the same sign are sought from each TP to make their effects complementary. 

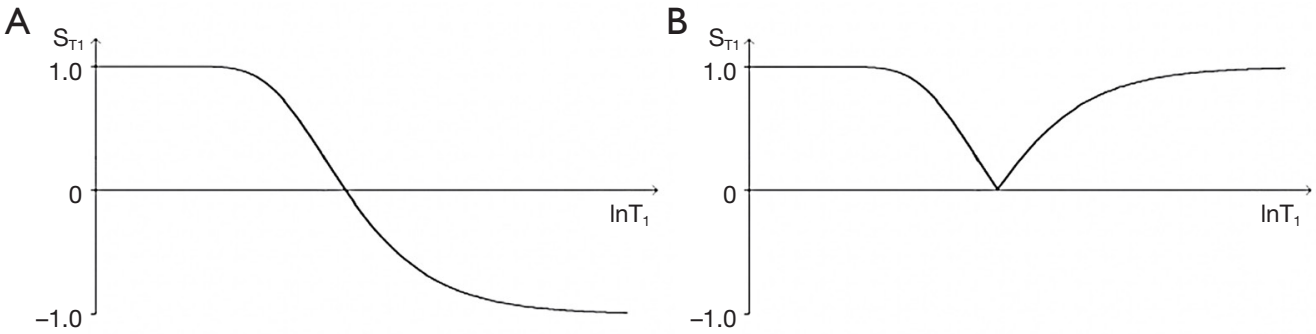

Figure 5 Long TR IR sequence $T_{1}$-filters with ps (A) and $m(B)$ reconstructions. The $T_{1}$-filter in (A) has high and low plateaus with a sloping region between. It has twice the range $(+1$ to -1$)$, and twice the slope of the corresponding $S E T_{1}$-filter. The $T_{1}$-filter in (B) is the same as (A) up to the zero, or null point. Beyond this, it is a mirror reflection across the $\mathrm{X}$ axis of the curve shown in (A). The slope of the filter shown in (A) is negative, and that shown in (B) is negative up to the null point after which it becomes positive. TR, repetition time; IR, inversion recovery; SE, spin echo; ps, phase sensitive; $\mathrm{m}$, magnitude.
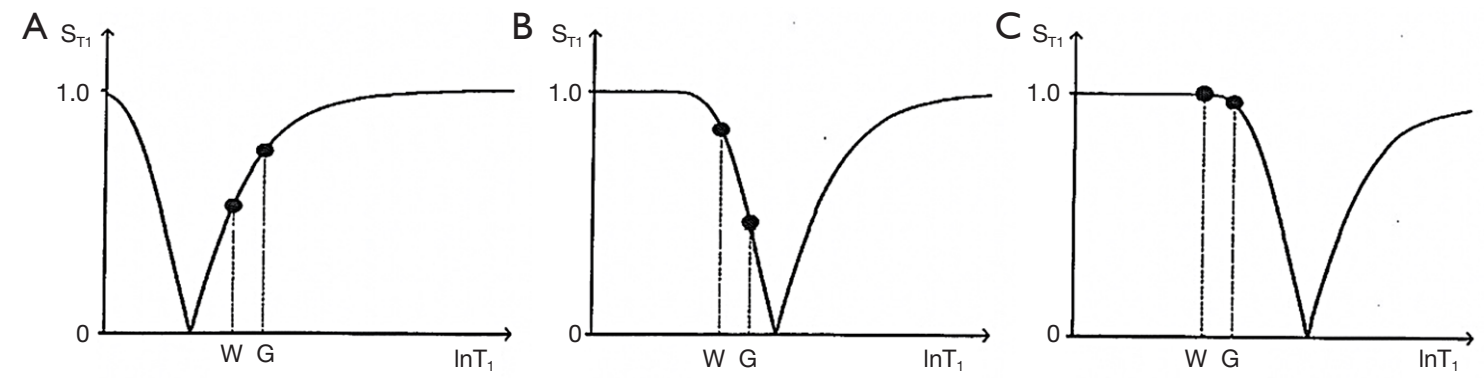

Figure 6 The long TR IR sequence. Plots of $S_{T 1}$ against $\ln T_{1}$ of the $I_{R} T_{1}$-filters for short $T_{s}(A)$, intermediate $T_{i}(B)$, and long $T I_{1}(C) I_{R}$ sequences. The positions of white matter $(W)$ and gray matter $(G)$ of the brain along the $X$ axis are shown, and are fixed in (A), (B) and (C). In (A) (the STIR sequence), $G$ is higher signal than $W$. $I n(B)$ (the intermediate $\mathrm{TI}_{\mathrm{i}}$ sequence) the filter is shifted to the right and $W$ is higher signal than $\mathrm{G}$, and in $(\mathrm{C})$, the long $\mathrm{TI}_{1}$ filter (e.g., the $\mathrm{T}_{2}$-FLAIR sequence) the filter is further shifted to the right and $W$ is slightly higher signal than G. These are the three main classes of the long TR IR sequence. The slope of the filter between $W$ and $G$ which is the sequence weighting is highly positive in (A), highly negative in (B), and mildly negative in (C). TR, repetition time; IR, inversion recovery; SE, spin echo; STIR, short inversion time inversion recovery; FLAIR, FLuid Attenuated inversion recovery.

The IR sequence has an additional $T_{1}$ filter (segment) to those of the SE sequence shown in Figure 4 for which:

$$
\mathrm{S}_{\mathrm{T} 1}=\left(1-2 \mathrm{e}^{-\mathrm{TI} / \mathrm{T1}}\right)
$$

This $\mathrm{T}_{1}$ filter is shown in phase-sensitive (ps) reconstructed form in Figure $5 \mathrm{~A}$ and in magnitude (m) reconstructed form in Figure $5 B$. When TI is increased, the filter shifts to the right (Figure 6). Figure 6A shows the IR $\mathrm{T}_{1}$-filter with a short $\mathrm{TI}_{\mathrm{s}}$ (e.g., the STIR sequence) where gray matter $(\mathrm{G})$ is higher signal than white matter (W) and the slope of the filter between P and Q is strongly positive. When TI is increased in Figure $6 B$ with $\mathrm{W}$ and $\mathrm{G}$ fixed in the same position on the $X$ axis, $W$ is now higher signal than $\mathrm{G}$ and the slope of the filter between $\mathrm{P}$ and $\mathrm{Q}$ is strongly negative. This is the intermediate $\mathrm{TI}_{\mathrm{i}} \mathrm{IR}$ sequence. When $\mathrm{TI}_{\mathrm{i}}$ is increased further to become TI long (TIl) in Figure $6 C, \mathrm{~W}$ is slightly higher than $\mathrm{G}$ and the slope of the filter is still negative but of much smaller magnitude than in Figure $6 \mathrm{~B}$. The sequence weighting which is the slope or first partial derivative of the filter is highly positive in (Figure $6 A$ ), highly negative in (Figure $6 B$ ) and slightly negative in (Figure 6C) using a short $\mathrm{TI}_{\mathrm{s}}$ (Figure 6A), an intermediate $\mathrm{TI}_{\mathrm{i}}$ (Figure $6 B$ ) and a long $\mathrm{TI}_{1}$ (Figure $6 C$ ) respectively. When $\mathrm{TR} \gg>\mathrm{T}_{1}$ the other $\mathrm{T}_{1}$ filter $\left(1-\mathrm{e}^{-\mathrm{TR} / \mathrm{T} 1}\right)$ becomes $\sim 1$ and the main determinant of contrast is the $\left(1-2 \mathrm{e}^{-\mathrm{TL} / \mathrm{T} 1}\right)$ filter.

For diffusion using the PGSE sequence an additional segment is added to those shown in Figures 2-6 for the SE sequence. This is the $\mathrm{D}^{*}$-filter which is an exponential decay where: 


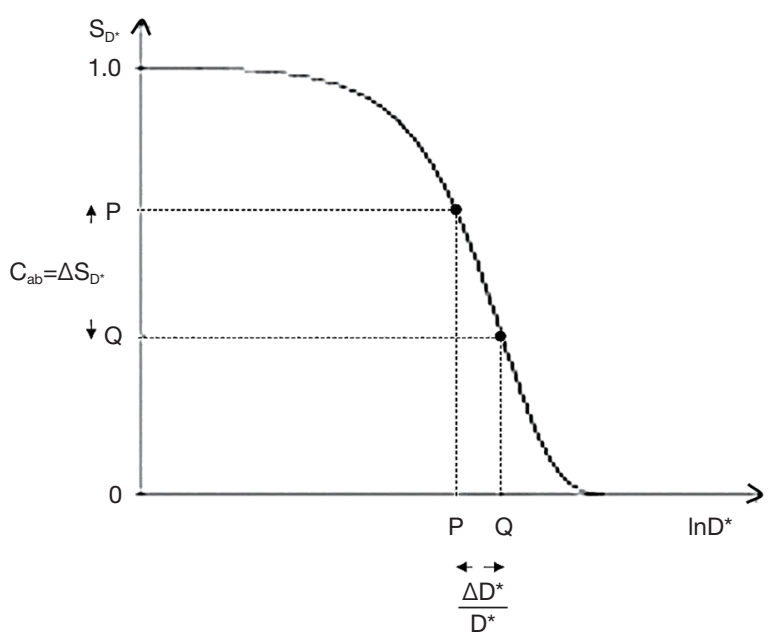

Figure 7 The $\mathrm{D}^{*}$-filter of the PGSE sequence. Plot of $\mathrm{S}_{\mathrm{D}^{*}}$ against $\ln \mathrm{D}^{*}$. Positive change in $\mathrm{D}^{*}$ from $\mathrm{P}$ to $\mathrm{Q} \Delta \mathrm{D}^{*} / \mathrm{D}^{*}$ along the $\mathrm{X}$ axis produces negative change from $\mathrm{P}$ to $\mathrm{Q} \Delta \mathrm{S}_{\mathrm{D}^{*}}$ along the $\mathrm{Y}$ axis. This is $C_{a b}$. The filter is a negative exponential with a negative slope. $C_{a b}$ is maximized where $b=1 / \mathrm{D}^{*}$. Increase in TE increases $b$ and shifts the filter to the left. PGSE, pulsed gradient spin echo.

$$
\mathrm{S}_{\mathrm{D} *}=\mathrm{e}^{-\mathrm{bD} *}
$$

$\mathrm{S}_{\mathrm{D}^{*}}$ is the diffusion filter signal, and $\mathrm{b}$ is the diffusion sensitivity parameter (Figure 7). The slope of the filter, i.e., its sequence weighting is negative. The positive change from $\mathrm{P}$ to $\mathrm{Q} \Delta \mathrm{D}^{*} / \mathrm{D}^{*}$ along the $\mathrm{X}$ axis shown in Figure 7 produces a negative change $\Delta \mathrm{S}_{\mathrm{D}^{*}}$ from $\mathrm{P}$ to $\mathrm{Q}$, and the negative contrast $\mathrm{C}_{\mathrm{ab}}$ along the $\mathrm{Y}$ axis. Maximum $\mathrm{D}^{*}$ weighting is achieved where $b^{*}=1$ using $C_{a b}$ and a $\ln X$ axis.

Significant $\mathrm{D}^{*}$-weighting requires a long TE with the PGSE sequence for tissues using present day clinical scanners. This is to provide time in the sequence for the two pulsed diffusion gradients applied before and after the $180^{\circ}$ pulse. This follows the $90^{\circ}$ excitation pulse, and precedes the data collection (dc) of the sequence. The long TE creates $\mathrm{T}_{2}$-weighting and so the sequence simultaneously has positive $T_{2}$-weighting (positive slope of the filter as shown in Figure 3) and negative $\mathrm{D}^{*}$-weighting (negative slope of the filter shown in Figure 7). It is therefore both positively $\mathrm{T}_{2^{-}}$ weighted and negatively $\mathrm{D}^{*}$-weighted.

\section{History of contrast in clinical MRI}

A history of contrast in MRI relevant to the present work is included in the following section.

The first clinical studies of the brain with MRI were performed in 1980 using a Steady State Free Precession (SSFP) sequence in which contrast was determined by the ratio $T_{2} / T_{1}(5)$. Frequently in disease, including cerebral infarction, tumors and many other conditions, both $T_{1}$ and $T_{2}$ are increased and so the ratio $T_{2} / T_{1}$ changes relatively little. Although tumors could be identified with the SSFP sequence, the soft tissue contrast was generally low and much less than that of CT. The first MR images which showed soft tissue contrast that was superior to CT, were intermediate $\mathrm{TI}_{\mathrm{i}} \mathrm{IR}$ sequences $(\mathrm{TI}=1,400 \mathrm{~ms}$, TR $=400 \mathrm{~ms}$ at $0.15 \mathrm{~T})$. These showed far more lesions than CT did in multiple sclerosis (MS) (112 vs. 19) (6). Contrast was largely determined by the $\mathrm{T}_{1}$-filter (Figure $6 \mathrm{~B}$ ) during TI. Chronic MS lesions which showed an increase in $T_{1}$ appeared low signal. The negatively sloped $T_{1}$ filter (Figure 2) was opposed by the positively sloped $\rho_{\mathrm{m}}$ - and $\mathrm{T}_{2}$-filters (Figures 3,4). Changes in $\rho_{\mathrm{m}}$ are typically small and the $T_{2}$-filter was minimized by use of a short TE of about $5 \mathrm{~ms}$. This resulted in the dominant $T_{1}$-weighting of the sequence.

The long TE heavily $\mathrm{T}_{2}$-weighted $\mathrm{SE}$ sequence followed soon after and was first used clinically in $1982(7,8)$. Increased signal was demonstrated in lesions in a variety of disease in the brain (e.g., infarction, subacute hemorrhage, trauma, demyelination, tumors). The sequence employed a long TR to minimize the opposed $T_{1}$ contrast and a long TE to increase $T_{2}$ contrast for the common change in disease of a small increase in $\rho_{\mathrm{m}}$ and larger increases in $T_{1}$ and $\mathrm{T}_{2}$ (Figures 3,4 ).

Clinical contrast enhancement with the paramagnetic agent Gd-DTPA was first demonstrated in $1984(9,10)$. Paramagnetic agents decrease $T_{1}$ and $T_{2}$ as well as $T_{2}{ }^{*}$ when susceptibility effects are manifest. This is the opposite to the increase in $T_{1}$ and $T_{2}$ that is commonly seen in disease. With the intermediate $\mathrm{TI}_{\mathrm{i}}(\mathrm{TR}=1,400, \mathrm{TI}=400 \mathrm{~ms}$ at $0.15 \mathrm{~T})$ IR sequence, an increase in signal due to shortening of $\mathrm{T}_{1}$ was seen. The sequence $T_{2}$-weighting was minimized with a short TE of $5 \mathrm{~ms}$. In Figure 2, reduction in $\mathrm{T}_{1}$ from $\mathrm{Q}$ to $\mathrm{P}$ leads to an increase in signal from $\mathrm{Q}$ to $\mathrm{P}$ along the $\mathrm{Y}$ axis and positive contrast.

In the same year (1984), Dixon introduced chemical shift imaging to selectively image either protons in water or in fat as a function of their difference in chemical shift using asymmetrical and symmetrical SE sequences. Subtraction of out-of-phase images from in-phase images selectively demonstrated fat (11).

The year 1985 saw the introduction of the STIR and 

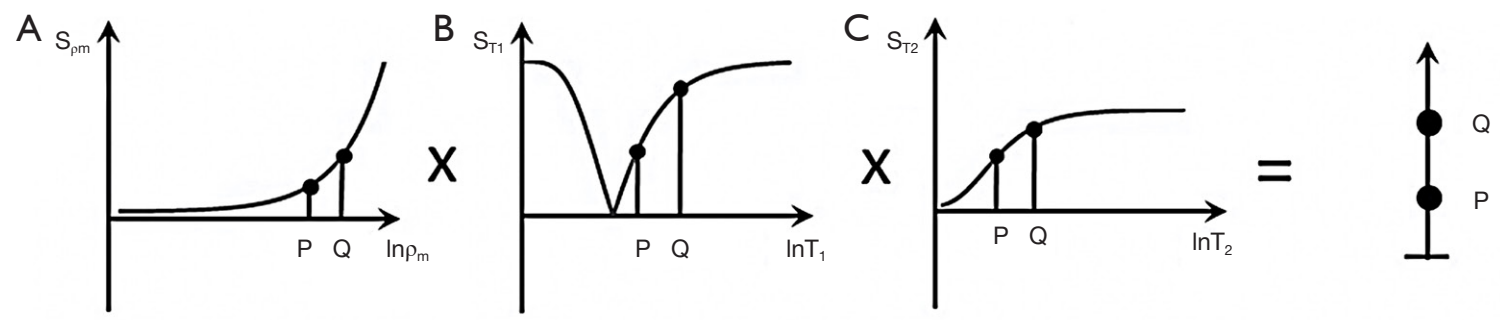

Figure $8 \mathrm{STIR}$ sequence (1985) (1). Plot of $\mathrm{S}_{\rho_{\mathrm{m}}} v s . \ln \rho_{\mathrm{m}}(\mathrm{A}), \mathrm{S}_{\mathrm{T} 1} v s . \ln \mathrm{T}_{1}$ using $\mathrm{m}$ reconstruction (B) and $\mathrm{S}_{\mathrm{T} 2} v s . \ln \mathrm{T}_{2}(\mathrm{C})$ for the short $\mathrm{TI}$ STIR sequence. The slopes of the three TP-filters from $\mathrm{P}$ to $\mathrm{Q}$ are all positive. Increase from $\mathrm{P}$ to $\mathrm{Q}$ in (A), (B) and (C) results in an increase in signal, i.e., positive contrast. The effect on overall contrast is synergistic with the contributions from the changes in $T_{1}$ and $T_{2}$ usually greater than that from $\rho_{\mathrm{m}}$. TI, inversion time; STIR, short inversion time inversion recovery.
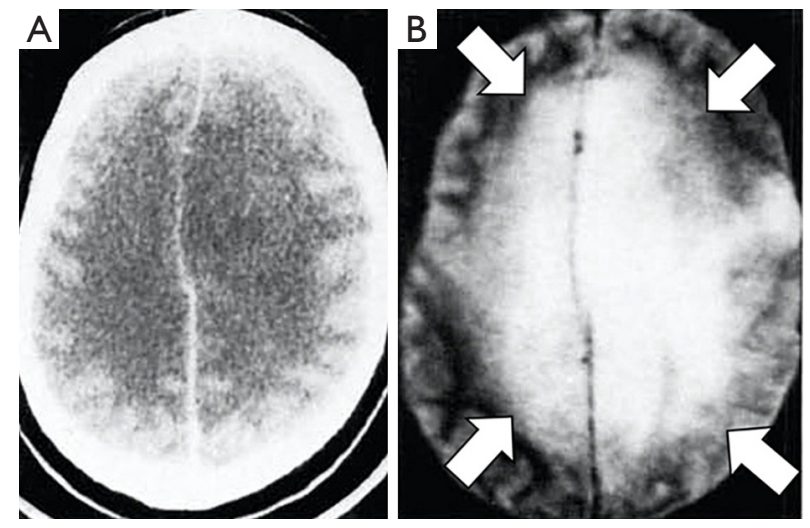

Figure 9 Contrast enhanced CT (A) and STIR (B) sequences (1985) (1). Frontoparietal astrocytoma grade II: contrast enhanced CT (A) and STIR (B) (TR =1,000 ms, TR =100 ms, TE =44 ms) scans (1985). The tumor shows a mass effect with displacement of the falx to the right in (A) but appears much more extensive in (B) (arrows). This includes extensive involvement of the right centrum semiovale. CT, computed tomography; STIR, short inversion time inversion recovery; TR, repetition time; TE, echo time.

DIR sequences which both showed synergistic $T_{1}$ and $T_{2}$ contrast for concurrent increases in $T_{1}$ and $T_{2}$ resulting in high signal lesions. Further synergistic contrast was created by nulling fat and CSF signals (1). In 1994, this was extended to include selective suppression of white or gray matter signals as well as CSF in and around the brain to exclusively see gray or white matter respectively (2).

In Figure 8, for the common change in disease of an increase in $\rho_{\mathrm{m}}, \mathrm{T}_{1}$ and $\mathrm{T}_{2}$ the contrast developed by each TP segment $\left(\rho_{\mathrm{m}}, \mathrm{T}_{1}\right.$ and $\left.\mathrm{T}_{2}\right)$ of the STIR sequence is positive and synergistic. This leads to high contrast demonstration of abnormalities in many conditions (e.g., Figure 9).
The STIR sequence gave an appearance in the body that looked like CT, but with abnormalities highlighted rather than showing lower signal due to a reduction in the X-ray attenuation on unenhanced CT scans (Figure 10). In addition, in the body and musculoskeletal system, the suppression of the fat signal from nulling produced further synergistic contrast (Figure 11). The STIR sequence was the archetypal synergistic contrast sequence.

The DIR sequence was also synergistic for increases in the major contrast determinants $T_{1}$ and $T_{2}$, although the second long $\mathrm{TI}_{1}$ segment reduces rather than increases $\mathrm{T}_{1}$ contrast. The long TI segment had the effect of multiplying the sequence by an additional term (Figure 12). The slope of the curve following white matter $(\mathrm{W})$ to the right in Figure $12 B$ is slightly reduced in Figure $12 C$ because the long $\mathrm{TI}_{1}$ segment in Figure $12 \mathrm{~A}$ produces slightly opposed contrast to that from the short $\mathrm{TI}_{s}$ segment in Figure 12B. However, the overall $T_{1}$ sequence weighting for small increases in $W$ is positive, and so is the $T_{2}$ sequence weighting. An image of a tumor obtained with a DIR sequence in 1985 is shown in Figure 13 (part B). A DIR image with nulling of white matter and CSF taken in 1994 is shown in Figure 14. Gray matter is seen without signal from white matter or CSF. The sequence is both positively $T_{1}$-weighted and $T_{2}$-weighted.

Diffusion weighted imaging was introduced into clinical practice in 1986 by Le Bihan et al. (12), but the key findings which established its clinical value were determined experimentally by Moseley in 1990. These were anisotropy in white matter (13), and increased signal in the brain in experimental acute infarction (14). The changes in this condition were due to a decrease in $\mathrm{D}^{*}$ starting within minutes of the infarction (e.g., Figure 15). This was followed later by an increase in $\mathrm{T}_{2}$, and these two effects were synergistic. With the PGSE sequence the weighting for $\mathrm{T}_{2}$ 

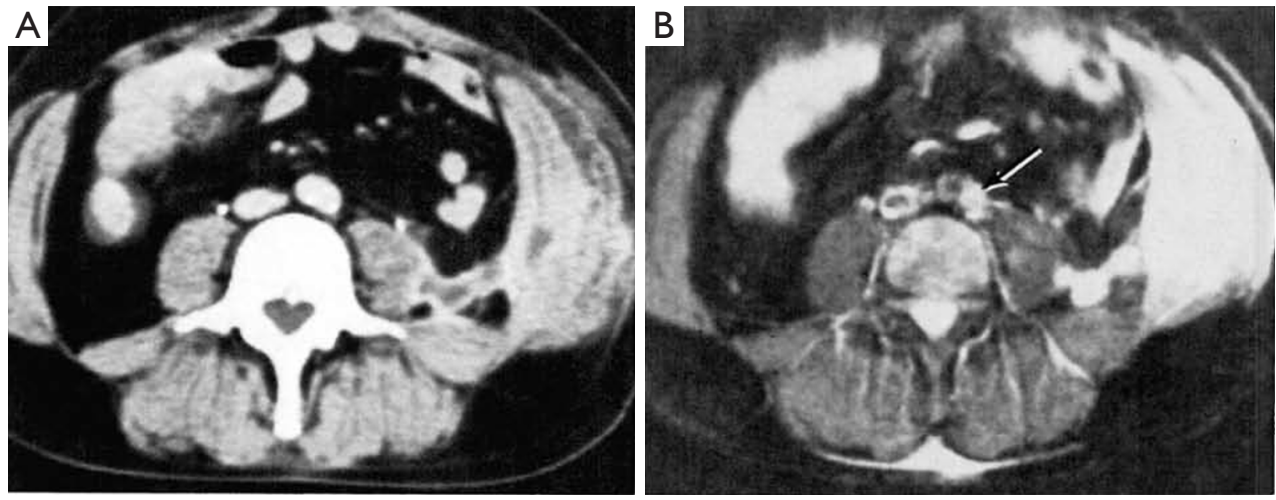

Figure 10 Psoas abscess. Contrast enhanced CT (A) and STIR (B) sequence (1985) (1). There is extensive involvement of the left abdominal wall in both (A) and (B). The psoas abscess is only seen on (B) (arrow). CT, computed tomography; STIR, short inversion time inversion recovery.
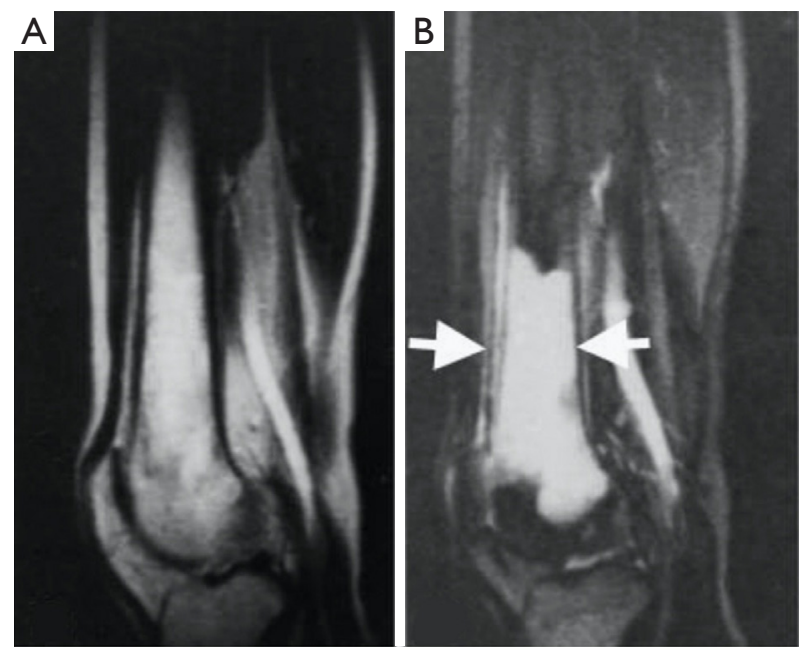

Figure $11 \mathrm{~T}_{1}$-weighted SE (A) and STIR (B) sagittal image of lymphoma involving the femur (1985) (1). The tumor is not easily differentiated from the yellow bone marrow in (A) but suppression of the signal from the marrow makes the tumor obvious in (B) (arrows). SE, spin echo; STIR, short inversion time inversion recovery.

is positive and that for diffusion is negative. If the change in $T_{2}$ is positive (i.e., an increase) this leads to positive $T_{2}$ contrast, and the negative change in $\mathrm{D}^{*}$ (i.e., a decrease) multiplied by the negative $\mathrm{D}^{*}$ sequence weighting leads to positive $\mathrm{D}^{*}$ contrast. This is synergistic with the positive contrast produced by the increase in $\mathrm{T}_{2}$.

Over time (days) in cerebral infarction the $\mathrm{D}^{*}$ reduction reverses and $\mathrm{D}^{*}$ increases. This produces negative $\mathrm{D}^{*}$ contrast which is no longer synergistic with the positive $T_{2}$ contrast and leads to a reduction in lesion conspicuity.

The diffusion weighting of the PGSE sequence produces a reduction in signal from $\mathrm{CSF}$ which has a high $\mathrm{D}^{*}$. This is an additional source of synergistic contrast.

Diffusion weighted imaging of an astrocytoma at this time (1991) (Figure 16) showed a high signal in the tumor consistent with an increase in $\mathrm{T}_{2}$ and a decrease in $\mathrm{D}^{*}$ with synergistic $T_{2}$ and $D^{*}$ contrast, as well as lower contrast in the surrounding vasogenic edema consistent with an increase in $T_{2}$ and an increase in $\mathrm{D}^{*}$ in the edema due to opposed $\mathrm{T}_{2}$ and $\mathrm{D}^{*}$ contrast (15). In another astrocytoma imaged at this time (Figure 17), reduced signal was seen in and around the tumor on the $\mathrm{D}^{*}$-weighted images (Figure $17 C, D)$ compared with the unsensitized images (Figure $17 A, B$ ) consistent with an increase in $\mathrm{T}_{2}$ and $\mathrm{D}^{*}$ in tumor and/or edema resulting in opposed $\mathrm{T}_{2}$ and $\mathrm{D}^{*}$ contrast. In the right corpus callosum little or no signal is seen with the diffusion gradient parallel to the white matter fibers which gives normal white matter a higher value of $\mathrm{D}^{*}$ (Figure $17 C$ ), but higher signal is seen in the corpus callosum (arrow) with the diffusion gradient perpendicular to the fibers (Figure $17 D$ ) where the normal value of $\mathrm{D}^{*}$ in white matter is lower so that the increased $\mathrm{D}^{*}$ in tumor and/or edema can become manifest.

Over a decade later in 2003, Takahara et al. (16) described DWIBS (Diffusion Weighted whole body Imaging with background Body Signal suppression) in which a STIR sequence was combined with a PGSE sequence using an echo planar imaging (EPI) dc. Body tumors were seen with very high positive contrast providing that their $\mathrm{D}^{*}$ was reduced which was the case in most tumors. For the 

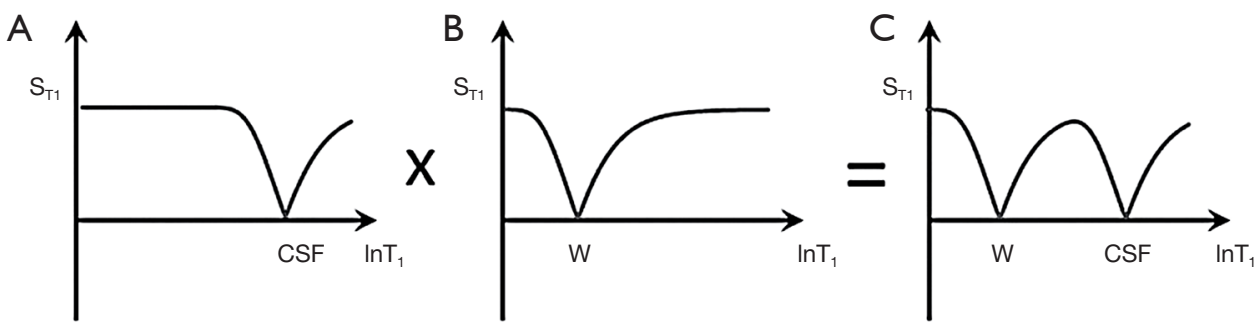

Figure 12 The DIR sequence. (A) is the $T_{1}$-filter nulling CSF, (B) is the $T_{1}$-filter nulling white matter $W$ and (C) is (A) multiplied by (B) in which both $W$ and CSF are nulled. The sequence weighting is the slope of the $T_{1}$-filter. It is zero, negative and positive for different values of T1. DIR, double inversion recovery; CSF, cerebrospinal fluid.
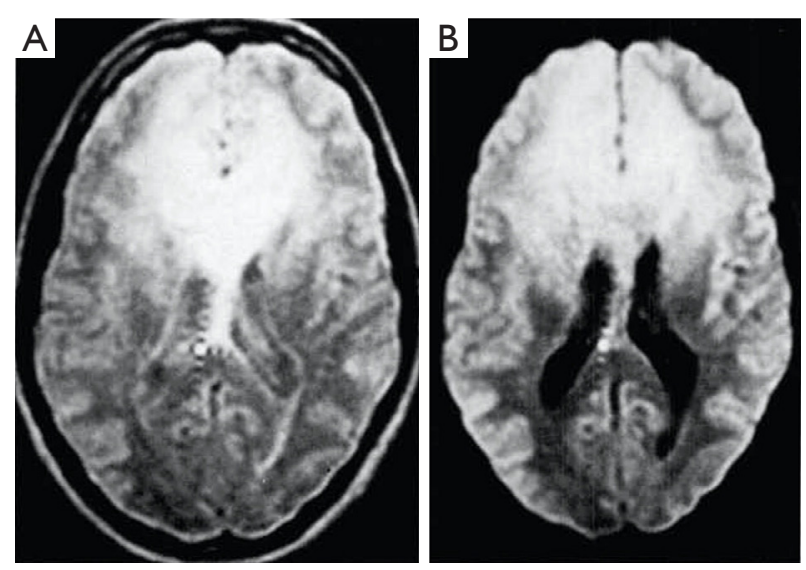

Figure 13 Glioma of the corpus callosum and frontal lobes (1985) (1). SE (TR =1,500 ms, TE =80 ms) (A) and DIR (TR $=3,000 \mathrm{~ms}, \mathrm{TI}_{1}=1,200 \mathrm{~ms}, \mathrm{TI}_{2}=100 \mathrm{~ms}$, TE $=44 \mathrm{~ms}$ ) (B) scans. The bilateral frontal tumor is seen on both scans. Both fat and CSF fluid signals are suppressed in (B). SE, spin echo; TR, repetition time; TE, echo time; TI, inversion time; DIR, double inversion recovery.

common change in tumors of $\uparrow \rho_{\mathrm{m}}, \uparrow T_{1}$ and $\uparrow T_{2}$ and $\downarrow D^{*}$ the contrast produced by each of these TPs with DWIBS is synergistic. This was coupled with fat signal reduction from the STIR component of the sequence, and fluid signal reduction because of the diffusion weighting of the sequence and the high value of $\mathrm{D}^{*}$ in fluids. The sequence was a classic example of scMRI.

Susceptibility weighted imaging was introduced in 1987 with demonstration of low signal areas in hemorrhage with a heavily $\mathrm{T}_{2}{ }^{*}$-weighted gradient echo sequence (17-19) and phase maps showing susceptibility differences. The effects were attributable to hemoglobin breakdown products with a reduction in $\mathrm{T}_{2}{ }^{*}$. The gradient echo sequences used a long TR which was insensitive to any concurrent decrease in $T_{1}$

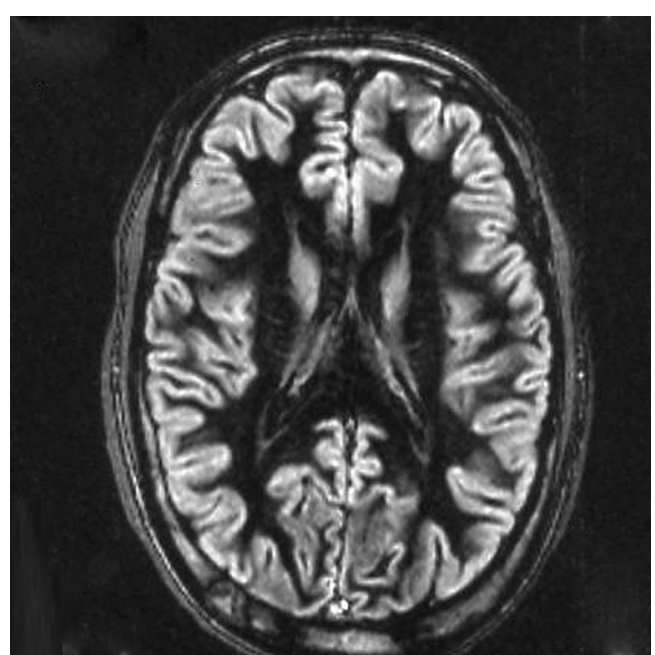

Figure 14 DIR scan of the brain nulling white matter and CSF (1994) (2). The DIR sequence shows gray matter with no signal from white matter or CSF. (courtesy FW Smith). DIR, double inversion recovery; CSF, cerebrospinal fluid.

due to methemoglobin or other iron breakdown products which would have increased signal. Later, Haacke combined the amplitude and phase images from the susceptibility weighted gradient echo images synergistically as double use of the single TP susceptibility to increase contrast (20).

MIOPs were introduced into clinical practice in 1989 and resulted in a general reduction in normal liver signal creating contrast with tumors and other lesions where the agent was not taken up and so their signal was not reduced (21).

The same principle was applied to lymph nodes where normal tissues took up the MIOPs and signal was reduced including metastatic and other pathologies which did not take up the agent.

MIOPs can also be used as blood pool agents to shorten 

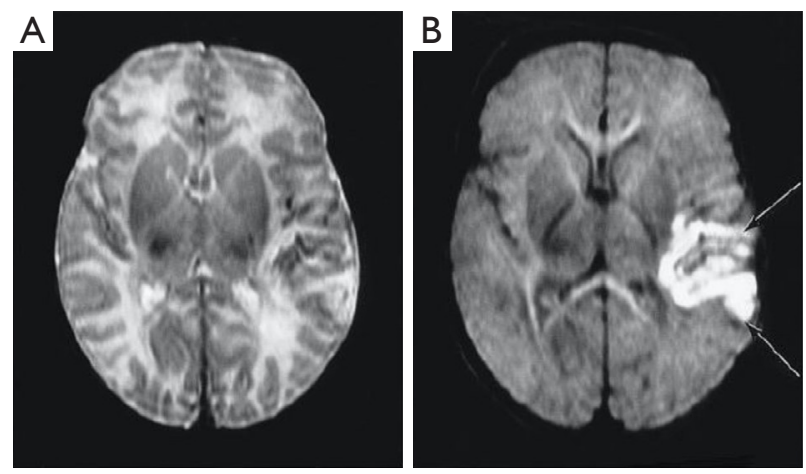

Figure 15 Acute neonatal infarction: $T_{2}$-weighted SE (A) and PGSE (B) scans. The infarction is just visualized in (A) but is obvious in (B) (arrows) as a consequence of its reduced D*. SE, spin echo; PGSE, pulsed gradient spin echo. the $T_{1}$ of blood for angiography. In these applications $T_{2} /$ $T_{2}{ }^{*}$ and $T_{1}$ are reduced. It is possible to synergistically increase contrast by using a $\mathrm{T}_{1}$-weighted UTE sequence with a second longer TE gradient echo and subtracting the second image from the first one (i.e., ES) so that the reduction in $\mathrm{T}_{2} / \mathrm{T}_{2}{ }^{*}$ produced by the MIOPs leads to positive $\mathrm{T}_{2} / \mathrm{T}_{2}{ }^{*}$ contrast which is synergistic with the positive contrast produced by the reduction in $T_{1}$ using the $\mathrm{T}_{1}$-weighted sequence.

In 1991 the basic blood oxygenation level-dependent (BOLD) effect was demonstrated in vivo (22). Brain activity is accompanied by an excessive increase in blood flow/perfusion leading to a decrease in paramagnetic deoxyhemoglobin and a consequent increase in $\mathrm{T}_{2}{ }^{*}$ which is typically detected with $\mathrm{T}_{2}{ }^{*}$-weighted EPI sequences and
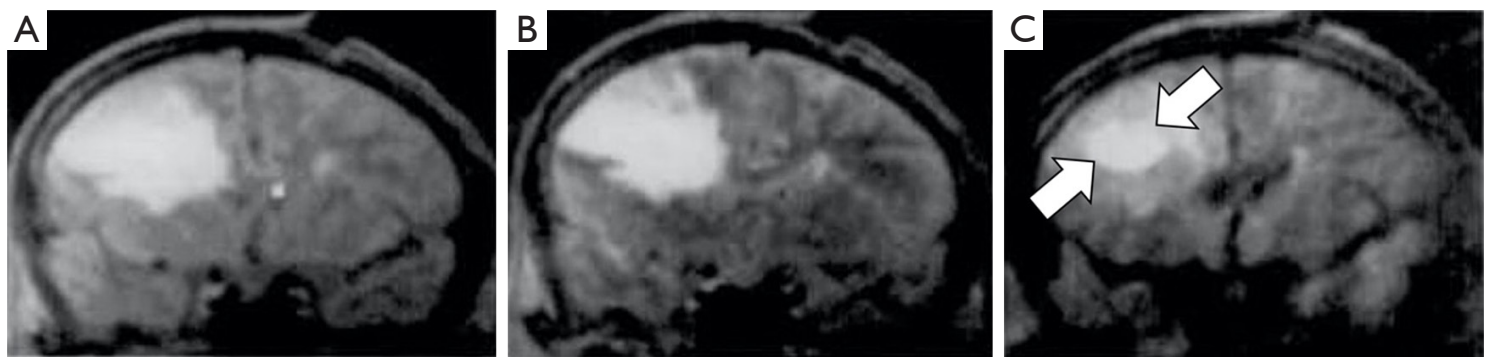

Figure 16 Probable metastasis (1991) (15). Coronal SE 1,500/80 ms (A), SE 1,500/130 ms (B) and SE 15,00/130 ms (b =550 s/mm²) $(C)$ scans. The central tumor and surrounding edema are high signal and isointense on the $\mathrm{b}=$ " 0 " image in (B). The central tumor is high signal on the diffusion sensitized image (C) (arrows) and the surrounding edema is lower signal. This is consistent with a reduced $\mathrm{D}^{*}$ for the tumor and an increased $\mathrm{D}^{*}$ for the edema. SE, spin echo.
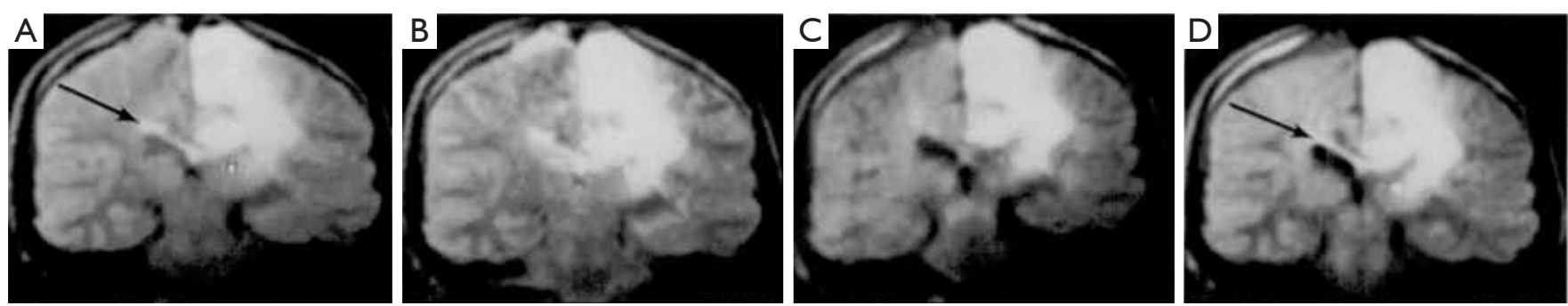

Figure 17 Left astrocytoma grade III extending to the right across the corpus callosum (1991) (15). SE 1,500/80 (A), SE 1,500/130 $\left(\mathrm{b}=" 0 " \mathrm{~s} / \mathrm{mm}^{2}\right)(\mathrm{B})$, PGSE 1,500/130 (b =550 s/mm gradient left to right) (C) and PGSE 1,500/130 (b =550 s/mm gradient anterior to posterior) (D) scans. The left hemispheric tissue is high signal without differentiation from associated vasogenic edema consistent with an increase in $\mathrm{D}^{*}$. The extension across the corpus callosum is seen in (A) (arrow), not well seen in (C) with gradients parallel to the corpus callosum fibers, but is apparent in (D) (arrow) with the gradient perpendicular to the corpus callosum fibers. This shows the effect of fiber anisotropy on the visualization of disease. The normal value of $\mathrm{D}^{*}$ is higher with fibers parallel to the gradient than across and this may overlap with abnormal values of $\mathrm{D}^{*}$. SE, spin echo; PGSE, pulsed gradient spin echo. 
results in an increase in tissue signal. Typical sequences are not sensitive to $T_{1}$ and therefore to any concurrent increase in tissue $T_{1}$ associated with an increase in the proportion of blood within tissue linked to increased perfusion.

The FLAIR (FLuid Attenuated IR, later $\mathrm{T}_{2}$-FLAIR) sequence was introduced in 1992. It increased the TE of the conventional $\mathrm{T}_{2}$-weighted $\mathrm{SE}$ sequence to increase its $\mathrm{T}_{2}$ sensitivity and used an inversion pulse to null the signal from CSF (23). In the brain, for white and gray matter the major contrast determinant is $T_{2}$ with $T_{1}$ contrast slightly negative (Figure 6C) for the common change in disease of an increase in $\rho_{\mathrm{m}}, \mathrm{T}_{1}$ and $\mathrm{T}_{2}$. The increased $T_{2}$ contrast is negated to some degree by the opposed $T_{1}$ contrast. The negative $T_{1}$-weighting is much higher for longer $T_{1}$ tissues, and very long $T_{1}$ CSF than it is for white and gray matter (Figure 6C). It produces contrast enhancement with intravenous GBCAs in long $\mathrm{T}_{1}$ lesions such as cerebral infarction (24). These lesions usually also have increased $\mathrm{T}_{2} \mathrm{~S}$ resulting in synergistic contrast from the decrease in $\mathrm{T}_{1}$ produced by the GBCAs. The negative $\mathrm{T}_{1}$ sequence weighting and reduction in $\mathrm{T}_{1}$ also result in marked increase in CSF signal when hemoglobin or molecular $\mathrm{O}_{2}$ is dissolved in CSF and decreases its $\mathrm{T}_{1}$.

MT received widespread clinical attention in 1992 (25) although it was described previously in 1983 (26). The MT pulse results in a reduction in the observed $\rho_{\mathrm{m}}$ and an equal fractional reduction in the observed $T_{1}$ of the free pool (27). The reduction in observed $\rho_{\mathrm{m}}$ can be synergistic with the reduction in observed $T_{1}$ in TI segments of STIR and DIR sequences and so further reduce the signal from normal tissue.

In many diseases MT effects are reduced so that in abnormal tissues there is less reduction in $\rho_{\mathrm{m}}$ and $\mathrm{T}_{1}$ leading to increased signal in the abnormality relative to normal tissue.

With the long TR IR sequence MT pulses can be applied in either or both of the TR and TI segments. The long TR segments minimize the $T_{1}$ effect so the dominant result is due to a reduction in $\rho_{\mathrm{m}}$. This can be added to the reduction in $\rho_{\mathrm{m}}$ and $T_{1}$ as another example of synergistic contrast. Synergistic contrast in angiography can be achieved with i.v. GBCAs shortening the observed T1 of blood and MT reducing the observed $\rho_{\mathrm{m}}$ of adjacent tissue. Fat and fluids are largely unaffected by MT so subtraction of a sequence with MT from one without MT reduces the signals from both these tissues. The MT ratio subtracts signals in this way and divides them by the signal of the sequence without MT. It typically includes the $\rho_{\mathrm{m}}$ and $\mathrm{T}_{1}$ effects which may be opposed.

The UTE sequence was introduced into clinical practice for lung imaging in 1992 (28) and has been more widely used since, including applications in the musculoskeletal system and brain (29). In tissues with a minority of ultrashort $\mathrm{T}_{2}$ components it is necessary to employ a mechanism to suppress the more abundant long $\mathrm{T}_{2}$ components in order to permit selective imaging of the ultrashort $\mathrm{T}_{2}$ components. This can be achieved by use of a long adiabatic inversion pulse to invert and null long $\mathrm{T}_{2}$ components while ultrashort $\mathrm{T}_{2}$ components are saturated by the pulse and then recover. Another mechanism to suppress long $T_{2}$ signals is ES. Adiabatic inversion and nulling, and ES provide two different ways of achieving synergistic $T_{2}$ contrast for increases in $T_{2}$ in, or from, the ultrashort $T_{2}$ range.

Another approach to specific imaging of tissues is to use the multi-exponential decay of $M_{x y}$ to separate different tissue components as a function of their $\mathrm{T}_{2} \mathrm{~s}$. Using this approach, it is possible to distinguish water associated with myelin $\left(\mathrm{T}_{1} \sim 400 \mathrm{~ms}, \mathrm{~T}_{2} / \mathrm{T}_{2}{ }^{*} \sim 10-20 \mathrm{~ms}\right)$ from axonal, extracellular and intracellular water in the brain ( $\mathrm{T}_{1} \sim 800 \mathrm{~ms}, \mathrm{~T}_{2} \sim 60 \mathrm{~ms}$ ) and provide selective imaging of myelin water as a myelin water fraction (MWF) map $(30,31)$.

Mugler et al. described the magnetization-prepared rapid gradient echo (MP-RAGE) sequence in 1990 (32) and from this was derived the MP2RAGE sequence in 2010 (33). This is an IR sequence which employs short and long TIs in two different segments. These are multiplied together and normalized. This reduces or eliminates $\rho_{\mathrm{m}}$ and $\mathrm{T}_{2}$ effects. The sequence produces synergistic negative $T_{1}$ contrast from both TI segments using differences in $\mathrm{T}_{1}$ twice. It shows higher gray-white matter contrast in the brain than MP-RAGE sequences and is another example of scMRI.

An additional sequence in this family is MPnRAGE (described in 2016) which employs a sliding TI window to obtain multiple IR images with different TIs primarily for $\mathrm{T}_{1}$ quantitation (34). The MOdified Look-Locker Inversion recovery (MOLLI) sequence can be used for the same purpose (35).

In angiography high signal from inflowing blood in one sequence may have subtracted from it low signal from blood that has been dephased in another registered sequence producing synergistic contrast by double use of the single tissue or fluid property blood flow (36,37).

Holstila $e t$ al. used a STIR sequence to null shorter $\mathrm{T}_{1}$ white adipose tissue (WAT) and selectively image the fat content of longer $T_{1}$ BAT using Dixon subtraction (38). This was synergistic use of the TPs $T_{1}$ and chemical shift, $\delta$.

An improved STIR (iSTIR) sequence was described in 2014 (39). In addition to the usual fat suppression, flowing 
blood signal was suppressed by dephasing, and ES was used to reduce long $T_{2}$ fluid signals. Thus, the STIR synergistic contrast was supplemented by further synergistic contrast from reduction in high signal blood and long $\mathrm{T}_{2}$ fluid signals to assist in visualizing high signal lesions.

The SIR sequence with subtraction of images with different TIs was described in 2017 (40) and used to study suprasellar tumors in 2018 (41).

\section{Clinical protocols}

These are usually established de facto or as a deliberate exercise by interested organizations or groups. They often include recommendations for specific pulse sequences, planes, spatial resolution, use of contrast agents and other factors, and are designed to be practical and time efficient. The protocols may be extended to include image interpretation and grading systems as well as a glossary of terms, lexicon or standardized terminology and frequently include illustrated examples.

They are often relatively conservative, based on clinical experience and aim to be implemented on a wide variety of imaging platforms.

The consensus groups developing these protocols may include surgeons and physicians to improve communication of findings. They often include rating or grading systems to place the imaging findings in a clinical context. Validation is both through experience and formal studies. Pulse sequences are only one aspect of this approach but are critical in establishing clinical effectiveness.

\section{MASTIR pulse sequences $(3,4)$}

A classification of MASTIR sequences is shown in Figure 18. They are divided into: (i) multiplied, (ii) added, (iii) subtracted, and (iv) FiTted groups.

\section{(I) Multiplied IR (MIR) sequences}

Historically, the first MASTIR sequence was described in 1985 as a DIR in which a long TI segment to null CSF was multiplied by a short TI segment to null fat using $\mathrm{m}$ reconstruction (1). This was extended to include nulling of white or gray matter as well as CSF in 1994 (2).

The MP2RAGE sequences also multiplies a long $\mathrm{TI}_{1}$ segment (e.g., TI =2,200 ms at 3T) by a short TIs segment (e.g., TI $=800 \mathrm{~ms}$ at $3 \mathrm{~T}$ ). The sequence is normalized and optimized for maximum contrast between white matter, gray matter and CSF (33).

\section{(II) Added IR (AIR) sequences}

The AIR sequences use a single TI with images reconstructed in ps and $\mathrm{m}$ form. Addition of these two images shows shorter $T_{1}$ tissues and suppresses the signal from longer $T_{1}$ tissues and fluids. The AIRES sequence supplements this by ES and so adds a $\mathrm{T}_{2}$-filter reducing the signal from longer $\mathrm{T}_{2}$ tissues and fluids to provide a combined short $T_{1}$ short $T_{2}$-filter. The Subtracted AIR (SAIR) sequence subtracts a longer TI image from a shorter one to selectively show a specific range of short $T_{1}$ tissues.

\section{(III) Subtracted (SIR) sequences}

Eight subgroups of SIR sequences are included in Figure 18. The first five use subtraction of a longer TI image from a shorter TI one (or vice versa, the reversed $r$ form). These start with the basic sequence (SIR) and add $\mathrm{T}_{2}$-weighting to it as the SIR echo subtraction (SIRES) sequence, and then add $\mathrm{D}^{*}$-weighting to this as the subtracted IR diffusion echo subtraction (SIRDES) sequence. The SE segment of the SIRES sequence is substituted by a gradient echo to produce the subtraction IR gradient echo subtraction (SIRGES) sequence. This can have added to it diffusion weighting as the SIRDGES sequence.

The sixth group includes double-echo sliding inversion recovery (DESIRE) which uses a sliding TI window to obtain many IR images with different TIs followed by a UTE dc and ES, and the short TR adiabatic pulse prepared inversion recovery echo subtraction (STAIRES) sequence. This sequence multiplies a very short TR segment by an intermediate $\mathrm{TI}_{\mathrm{i}}$ segment to reduce to zero, or nearly zero, long $T_{2}$ signals from tissues and fluids with a wide range of $T_{1}$ s. It is used with UTE dcs to allow selective imaging of ultrashort $T_{2}$ tissues. This is followed by ES to reduce to zero the signal from any long $\mathrm{T}_{2}$ tissues which are not completely nulled. Both the DESIRE and STAIRES sequences are used to selectively image myelin and other ultrashort $\mathrm{T}_{2}$ tissues.

The seventh group uses the same TI and subtracts a ps image from an m image once ( $\left.S^{1} I R\right)$, or twice ( $\left.S^{2} I R\right)$ when different TIs are used, for example, to selectively show a fluid or tissue.

The eighth group of sequences is a basic IR echo subtraction (IRES), and the STIRES sequence which nulls shorter $T_{1}$ WAT and uses Dixon subtraction of out-ofphase images from in-phase images to selectively show the 


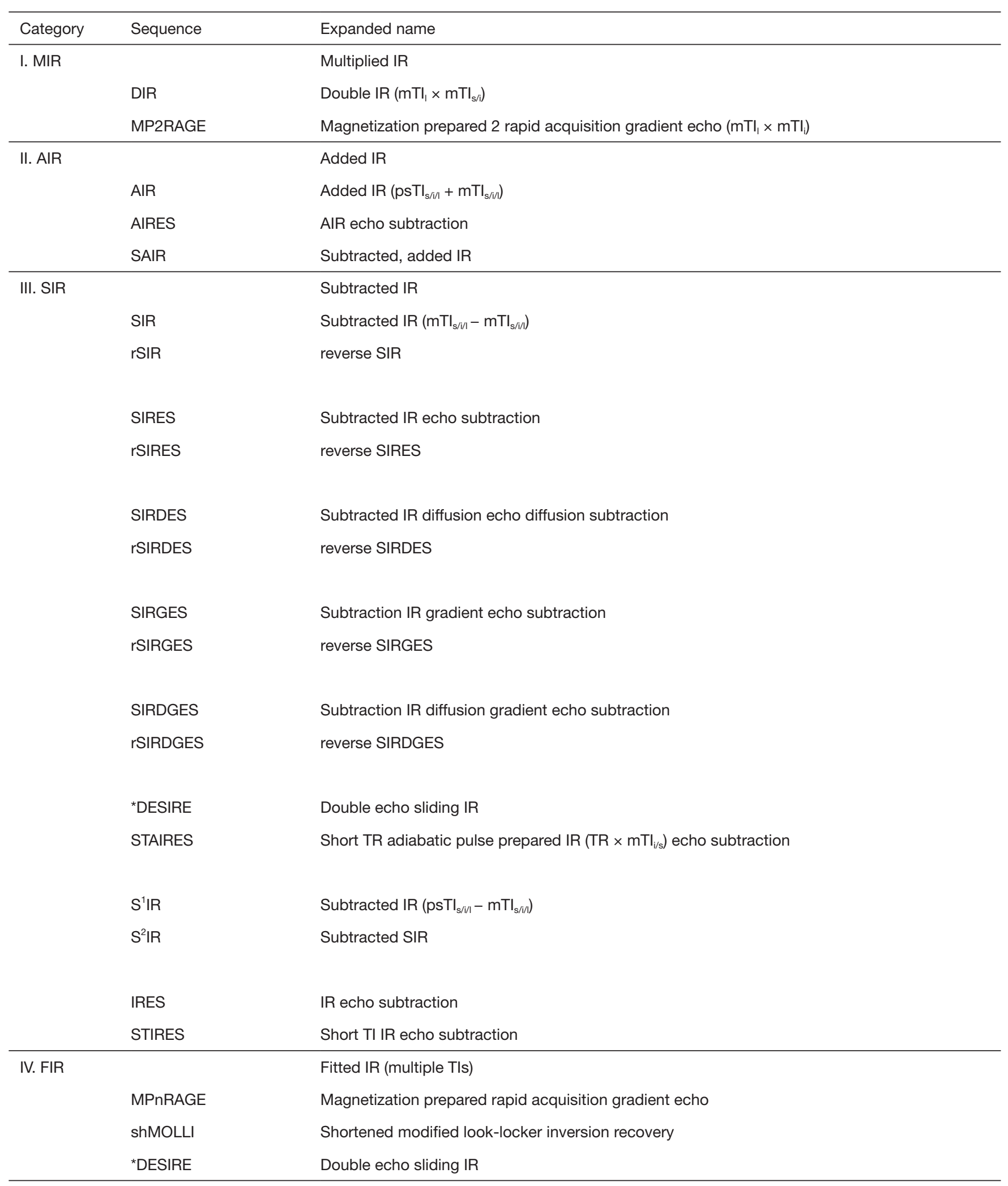

Figure 18 MASTIR sequences: classification. *, included in both the subtracted and fitted categories. 
Table 1 Conventional and MASTIR sequences, mainly for sensitivity

\begin{tabular}{|c|c|c|c|c|}
\hline Sequence \# & Sequences: conventional; MASTIR & Target tissues/fluids & Common TP changes & Purpose \\
\hline I & T,W SE, MP-RAGE; SIR, rSIR & $\begin{array}{l}\text { White or gray matter or } \\
\text { both }\end{array}$ & $\uparrow$ in $\mathrm{T}_{1}$ & $\begin{array}{l}\text { Sensitivity to increase in } T_{1} \text { in common } \\
\text { disease in white or gray matter or both }\end{array}$ \\
\hline II & $\mathrm{T}_{2} \mathrm{~W}$ SE, $\mathrm{T}_{2}$-FLAIR; SIRES, rSIRES & $\begin{array}{l}\text { White or gray matter or } \\
\text { both }\end{array}$ & & $\begin{array}{l}\text { Sensitivity to increase in } T_{1} \text { and } T_{2} \text { in } \\
\text { common disease in white or gray } \\
\text { matter or both }\end{array}$ \\
\hline III & $\mathrm{T}_{2} \mathrm{~W}$ SE, D, $\mathrm{D}^{*}$ map; SIRDES & $\begin{array}{l}\text { White or gray matter or } \\
\text { both }\end{array}$ & $\uparrow$ in $\mathrm{T}_{1}, \mathrm{~T}_{2}$ and $\mathrm{D}^{*}$ & $\begin{array}{l}\text { Sensitivity to increase in } T_{1}, T_{2} \text { and } D^{*} \\
\text { in common disease in white or gray } \\
\text { matter or both }\end{array}$ \\
\hline IV & $\begin{array}{l}\mathrm{T}_{1} \mathrm{~W} \text { GE, } \mathrm{T}_{2} \mathrm{~W} \text { SE, } \mathrm{T}_{2} \text {-FLAIR pre and } \\
\text { post contrast enhancement with } \\
\text { Gd-DTPA; rSIR or rSIRES pre and post } \\
\text { contrast enhancement with Gd-DTPA }\end{array}$ & $\begin{array}{l}\text { White or gray matter or } \\
\text { both } \\
\text { t }\end{array}$ & $\begin{array}{l}\downarrow \text { in } \mathrm{T}_{1} \text { in lesions post } \\
\text { contrast }\end{array}$ & $\begin{array}{l}\text { Demonstration of contrast enhance- } \\
\text { ment due to Gd-DTPA }\end{array}$ \\
\hline
\end{tabular}

MASTIR, Multiplied, Added, Subtracted and/or FiTted; TP, tissue property; MP-RAGE, magnetization-prepared rapid gradient echo; SIR, subtracted inversion recovery; rSIR, reverse SIR; SE, spin echo; GE, gradient echo; FLAIR, FLuid Attenuated inversion recovery; SIRES, subtracted inversion recovery echo subtraction; rSIRES, reverse SIRES; SIRDES, subtracted inversion recovery diffusion echo diffusion subtraction.

lipid present in longer $\mathrm{T}_{1}$ BAT.

\section{(IV) FiTted IR (FIR) sequences}

These obtain multiple IR images primarily for quantification of $\mathrm{T}_{1}$ [MPnRAGE and shMOLLI $\left.(34,35)\right]$. The DESIRE sequence can be used in this way but can also be used for selecting the best TI to null long $T_{2}$ components in tissue or tissues with different $T_{1}$ s. The DESIRE sequence is included in both the subtraction and fitting categories.

\section{Protocols with MASTIR sequences}

\section{Brain and spinal cord}

The first four sequences described below are mainly focused on sensitivity and are listed in Table 1.

\section{(I) The SIR and reverse SIR (rSIR) sequences}

The SIR sequence can be understood using a univariate model and can provide nearly double the sequence weighting of standard IR sequences over a particular range of $\mathrm{T}_{1}$ values (Figure 19). The dynamic range is effectively adjusted to the minimum and maximum signals at the lower and upper ends of the $T_{1}$ range respectively. In setting this up it is necessary to (i) specify a starting value of $\mathrm{T}_{1}$ to define the first TI, (ii) nominate the direction(s) of the change $\Delta T_{1}$ (i.e., positive, negative or both) so that a SIR or rSIR sequence can be used to produce positive/negative contrast or both, (iii) decide on the range of $T_{1}$ values of interest to specify the second TI. The range can be chosen to detect just increases or decreases, or both, in $T_{1}$ within a single tissue (e.g., white or gray matter) or cover a broader range of $T_{1}$ values, e.g., to extend the $T_{1}$ sensitive range to cover both decreases in the $T_{1}$ of white matter and increases in the $T_{1}$ of gray matter. The wider range results in reduced $\mathrm{T}_{1}$ sequence weighting and so less contrast for the same change in $T_{1}$ (but broader coverage of changes in $T_{1}$ ). It is also possible to null gray matter with the first $\mathrm{TI}_{i}$ and use a longer $T 1_{1}$ for the second $T I$. This shows low gray-white matter contrast and can display lesions with an increased $\mathrm{T}_{1}$ as the highest signal on the image.

The rSIR filter (Figure 20) is used for decreases in $T_{1}$ to provide an increase in signal, e.g., for gray matter using TIs to null gray $\left(\mathrm{TI}_{\mathrm{i}}\right)$ and white (TIs) matter. Because the SIR sequence is only dependent on the single $\mathrm{TP} \mathrm{T}_{1}$, if the TIs are chosen so that normal tissue is in the mid-range of the sensitive region, then it is sensitive to both increases and decrease in $T_{1}$ and shows these as increases or decreases in signal. This avoids the complication of the $T_{1}$ contrast of different signs becoming opposed to that from other TPs when these are used, and so reducing the overall contrast.

The sequence is usually run with a short or UTE so there is little significant $T_{2}$-weighting. In any case the $T_{2}$ contrast is largely cancelled as a result of the subtraction.

The appearance of the brain is shown in Figure 21 for a TIs image nulling white matter (Figure 21A), a $\mathrm{TI}_{\mathrm{i}}$ nulling gray matter (Figure 21B), SIR (Figure $21 A$ minus Figure 21B) 


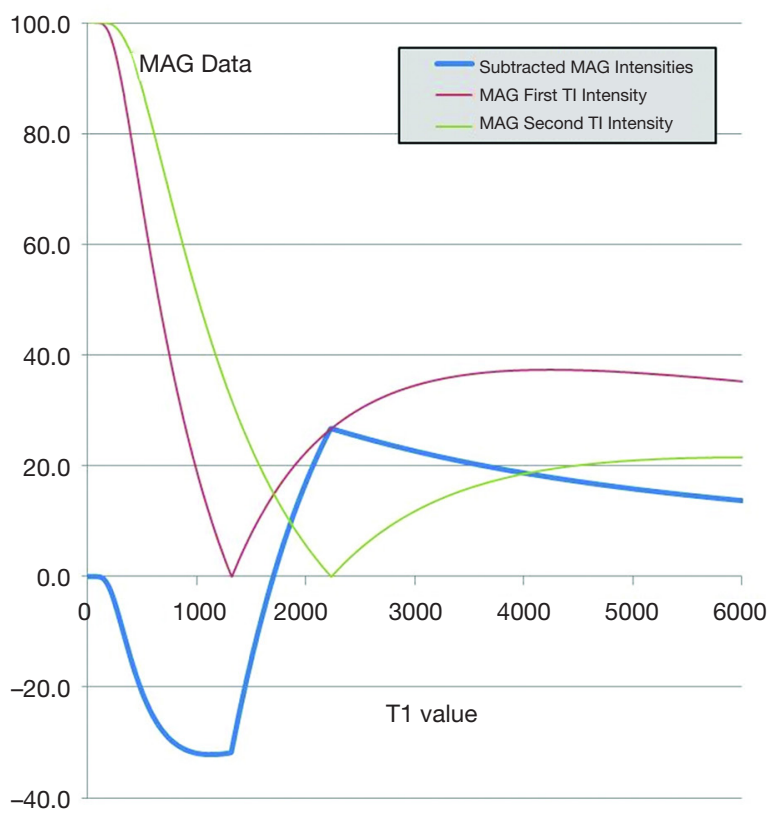

Figure $19 \mathrm{~T} 1$-filter for SIR $\left(\mathrm{mTI}_{\mathrm{s}}-\mathrm{mTI}_{\mathrm{i}}\right)$ filter. An $\mathrm{m}$ reconstructed intermediate $\mathrm{TI}\left(\mathrm{TI}_{\mathrm{i}}\right)$ (green image is subtracted from a $\mathrm{m}$ reconstructed short $\mathrm{TI}\left(\mathrm{TI}_{\mathrm{s}}\right)$ image (red). The difference image (blue) shows that for $\mathrm{T} 1_{s}$ in the range between the null points of the two $\mathrm{TI}_{s}$ there is a very high positive slope or sequence weighting. The slope of the subtracted curve in this range is greater than that of either of the two original images. This leads to high positive contrast for increases in $T_{1}$ values in the range between two nulling points of the short and intermediate TI sequences. SIR, subtracted inversion recovery; TI, inversion time; MAG, magnitude.
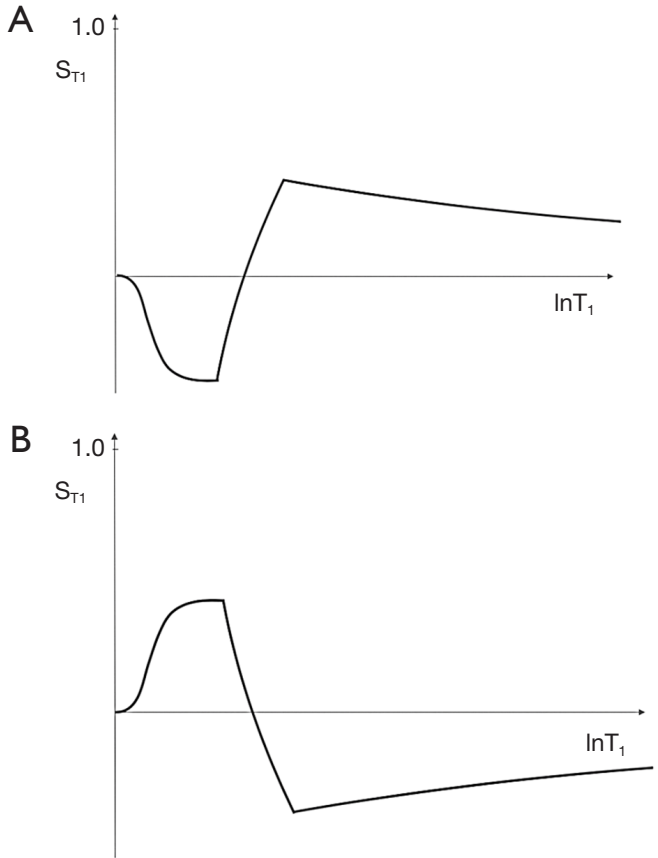

Figure $20 \mathrm{~T}$ 1-filter for SIR $\left(\mathrm{mTI}_{\mathrm{s}}-\mathrm{mTI}_{\mathrm{i}}\right)(\mathrm{A})$ and reversed $\mathrm{rSIR}$ $\left(\mathrm{mTI}_{\mathrm{i}}-\mathrm{mTI}_{\mathrm{s}}\right)(\mathrm{B})$. Between the null points for $\mathrm{TI}_{\mathrm{s}}$ and $\mathrm{TI}_{\mathrm{i}}$ the $\mathrm{T}_{1^{-}}$ filter in (A) has a marked positive slope while the $T_{1}$-filter in (B) has a marked negative slope. In (B) reductions in $T_{1}$ from the intermediate $\mathrm{TI}_{\mathrm{i}}$ null point produce marked increases in signal. This occurs with $\mathrm{T}_{1}$ shortening due to GBCAs or iron deposition. SIR, subtracted inversion recovery; TI, inversion time; rSIR, reversed subtraction inversion recovery; GBCAs, gadolinium-based contrast agents.
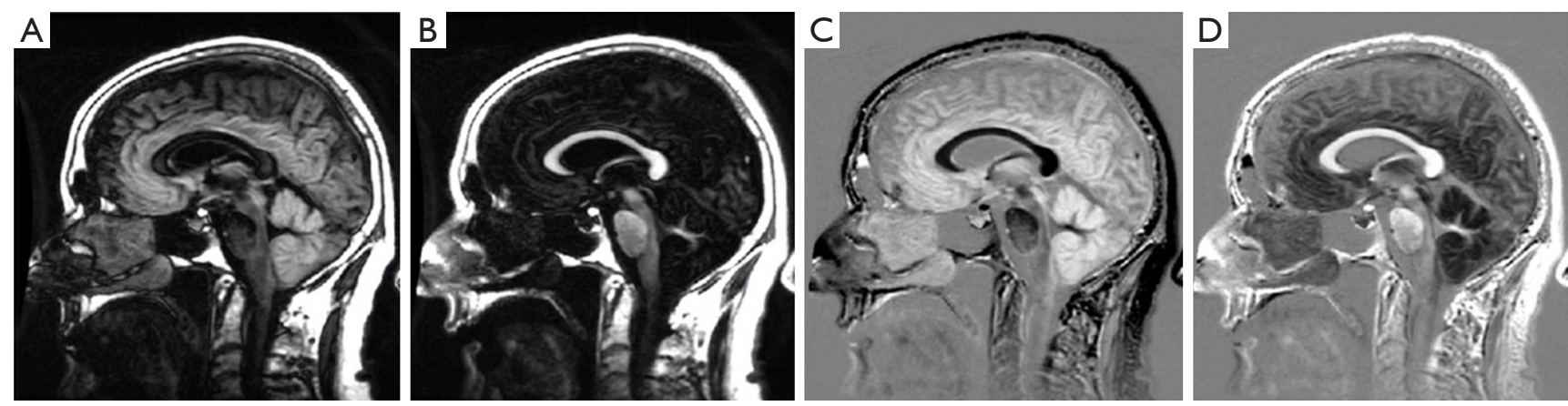

Figure 21 Sagittal images of a normal brain. $\mathrm{mTI}_{\mathrm{s}}$ nulling white matter $(\mathrm{A}), \mathrm{mTI}_{\mathrm{i}}$ nulling gray matter $(\mathrm{B}), \mathrm{SIR}\left(\mathrm{mTI}_{\mathrm{s}}-\mathrm{mTI}_{\mathrm{i}}\right)$ in $(\mathrm{C})$ and $\operatorname{rSIR}\left(\mathrm{mTI}_{\mathrm{i}}-\mathrm{mTI}_{\mathrm{s}}\right)$ in (D). There is high gray white matter contrast with gray matter high signal and white matter low signal in (C). In (D) the gray white matter contrast is reversed with white matter white, and gray matter black. CSF is of intermediate signal near the noise level in both (C) and (D). SIR, subtracted inversion recovery; TI, inversion time; rSIR, reversed subtraction inversion recovery; CSF, cerebrospinal fluid. 


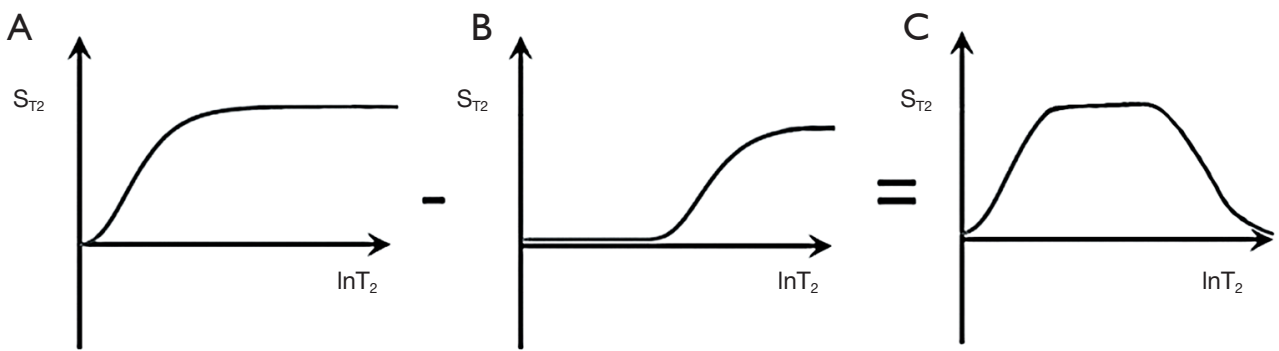

Figure 22 The echo subtraction (ES) $\mathrm{T}_{2}$-filter. Subtraction from an ultrashort TE image $\left(\mathrm{TE}_{\mathrm{u}}\right)$ of an intermediate $\mathrm{TE}_{\mathrm{i}}$. This results in a broad band pass filter. For medium $\mathrm{T}_{2}$ values the slope of the curve is negative and this is the reverse of the usual SE sequence weighting. TE, echo time.

in Figure $21 C$ and rSIR (Figure $21 B$ minus Figure 21A) in Figure 21D.

CSF shows a mid-range signal (Figure 21C,D). This can be reduced to zero (but is still in the mid-range of the subtraction image) using a Multiplied SIR (MSIR) filter with a long $\mathrm{TI}_{1}$ designed to null CSF. Compared with the $\mathrm{T}_{1}$-filter of a SE sequence the SIR sequence has up to four times the $T_{1}$ sequence weighting and up to twice that of the source IR sequences used for the subtractions. This can provide a higher degree of $T_{1}$ sequence weighting than seen with other clinically used sequences for $T_{1}$ changes in the range between the nulling points of the shorter and longer TI sequences.

The forward (default) subtraction SIR produces images with white matter low signal and gray matter high signal (Figure 21C). Pathology in white matter which increases $T_{1}$ results in higher signal than that in normal white matter which is similar to the appearances seen with conventional heavily $\mathrm{T}_{2}$-weighted $\mathrm{SE}$ sequences, but the contrast is due to increase in $T_{1}$, not increase in $T_{2}$.

The appearance of the rSIR sequences is white matter high signal and gray matter low signal which is similar to that of the traditional intermediate $\mathrm{TI}_{\mathrm{i}} \mathrm{IR}$ sequence except that the CSF signal is mid-range, not very low (Figure 21D).

(II) The SIRES and reverse SIRES (rSIRES) sequences While the SIR sequence can be understood using a univariate $T_{1}$ model, the SIRES sequences requires a multivariate model and combines the very high $\mathrm{T}_{1}$ sequence weighting of the SIR sequence with synergistic $\mathrm{T}_{2}$-weighting. This requires ES (Figure 22). The SIRES sequence is shown in Figure 23. The first part of the acquisition (row I) is performed with a short TIs. Assuming the change in TPs is that commonly seen in brain lesions which is an increase in each of $\rho_{\mathrm{m}}, \mathrm{T}_{1}$ and $T_{2}$, the TIs segment produces positive $T_{1}$ contrast. This is synergistic with the positive $T_{2}$ contrast produced by an increase in $T_{2}$ in the third segment. The second part (row II) is performed with an intermediate $\mathrm{TI}_{\mathrm{i}}$, as well as ES to produce negative $T_{1}$ and negative $T_{2}$ contrast. The second part (row II) is then subtracted from the first part (row I) in row III of Figure 23. This provides synergistic $\mathrm{T}_{1}$ and $T_{2}$ contrast compared to the dominant $T_{2}$-weighting with a $T_{2}$-weighted $S E$ sequence or $T_{2}$-FLAIR sequence. The $T_{2}$-weighted $\mathrm{SE}$ sequence has little or no $\mathrm{T}_{1}$ weighting and the $\mathrm{T}_{2}$-FLAIR usually has slightly opposed $\mathrm{T}_{1}$-weighting for white and gray matter, not synergistic $\mathrm{T}_{1}$-weighting.

There is little or no net $\rho_{\mathrm{m}}$ sequence weighting because the effects in the two parts of the SIRES sequence largely counteract one another.

The rSIRES sequence can be produced with an appearance like an intermediate $\mathrm{TI}_{\mathrm{i}}$ sequence (white matter high signal, gray matter low signal) but with combined $T_{1}$ and $T_{2}$ sequence weighting.

\section{(III) The SIRDES and rSIRDES sequence}

The SIRDES sequence extends the SIRES sequence by adding a $\mathrm{D}^{*}$ segment. The objective is to obtain synergistic $\mathrm{T}_{1}, \mathrm{~T}_{2}$ and $\mathrm{D}^{*}$ contrast for the changes in disease affecting all three of these TPs.

To do this it is necessary to perform ES, and DS where a $b=500-1,500 \mathrm{~s} / \mathrm{mm}^{2}$ diffusion image is subtracted from a $b=0-20 \mathrm{~s} / \mathrm{mm}^{2}$ image to reverse the $\mathrm{D}^{*}$ sequence weighting (Figure 24). CSF signal may need to be suppressed on the $b=0-20 \mathrm{~s} / \mathrm{mm}^{2}$ image using ES.

For a lesion which has an increase in $T_{1}$ and $T_{2}$, and a decrease in $\mathrm{D}^{*}$, row I in Figure 25 shows the contrast produced by each of these TPs. The increases from $\mathrm{P}$ to 

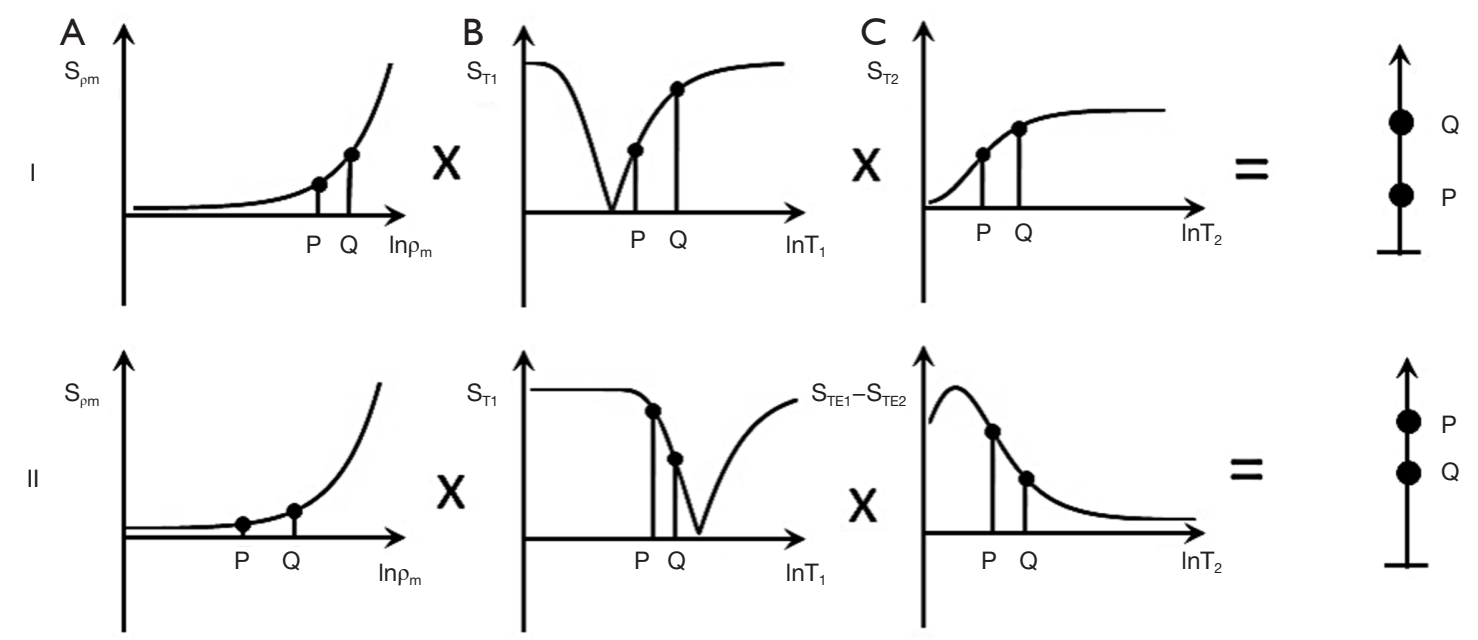

III

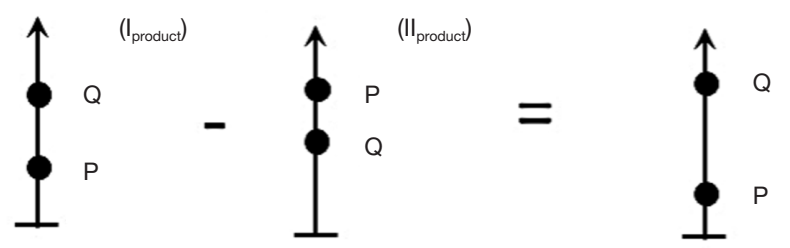

Figure 23 SIR echo subtraction (SIRES) sequence (multivariate model). The $\rho_{\mathrm{m}}, \mathrm{T}_{1}$ and $\mathrm{T}_{2}$-filters for a short TIs sequence are shown in the top row (I). For an increase in $\rho_{\mathrm{m}}, \mathrm{T}_{1}$ and $\mathrm{T}_{2}$ from $\mathrm{P}$ to $\mathrm{Q}$ this results in an increase in signal from $\mathrm{P}$ to $\mathrm{Q}$ in each filter, and multiplication of each of the filters shows high positive contrast from $\mathrm{P}$ to $\mathrm{Q}$ (right hand side). In the middle row (II) an intermediate $\mathrm{TI}_{\mathrm{i}}$ is used and the $\mathrm{T}_{1}$-filter sequence weighting from $\mathrm{P}$ to $\mathrm{Q}$ is negative. The $\mathrm{ES} \mathrm{T}_{2}$-filter $\left(\mathrm{S}_{\mathrm{TE1} 1}-\mathrm{S}_{\mathrm{TE} 2}\right)$ is also negative from $\mathrm{P}$ to $\mathrm{Q}$. The multiplied result shows negative contrast from $\mathrm{P}$ to $\mathrm{Q}$ (right hand side). The bottom row (III) shows normalized subtraction of the signals from $\mathrm{P}$ and $\mathrm{Q}$ in the middle row (II product) from the corresponding signals in the first row (I product). This creates higher positive contrast from $\mathrm{P}$ to $\mathrm{Q}$ than from the top row alone.
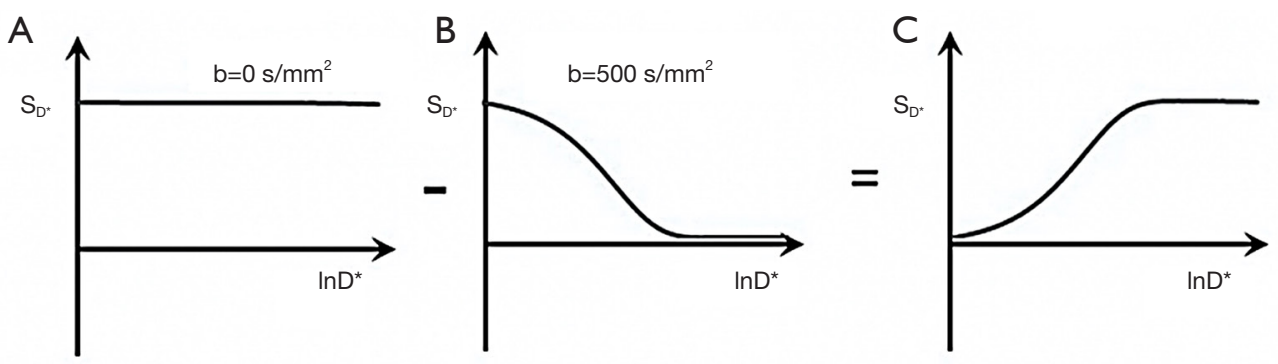

Figure 24 The diffusion subtraction (DS) $\mathrm{D}^{*}$-filter. Subtraction of the $\mathrm{b}=500 \mathrm{~s} / \mathrm{mm}^{2}$ filter (B) from the $\mathrm{b}=0 \mathrm{~s} / \mathrm{mm}^{2}$ filter (A) produces (C) which reverses the sequence weighting shown in (B) so that an increase in $\mathrm{D}^{*}$ increases the signal on the subtracted $\mathrm{D}^{*}$-filter in $(\mathrm{C})$. This can be used to make $D^{*}$ contrast synergistic with the contrast produced by increases in $\rho_{m}$ and $T_{2}$ when $D^{*}$ is increased.

$\mathrm{Q}$ in $\mathrm{T}_{1}$ with a short TIs (B) and in $\mathrm{T}_{2}$ from $\mathrm{P}$ to $\mathrm{Q}$ in $(\mathrm{C})$ produce positive contrast. The negative change in $\mathrm{D}^{*}$ from $\mathrm{P}$ to $\mathrm{Q}$ in (D) also produces positive contrast because of the negative slope of the $\mathrm{D}^{*}$ filter.

In the middle row (II) with an intermediate $\mathrm{TI}_{\mathrm{i}}$, the $\mathrm{T}_{1}$ change from $\mathrm{P}$ to $\mathrm{Q}$ produces negative $\mathrm{T}_{1}$ contrast in (B). The ES in (C) also results in negative $\mathrm{T}_{2}$ contrast for an increase in $\mathrm{T}_{2}$ from $\mathrm{P}$ to $\mathrm{Q}$. The decrease in $\mathrm{D}^{*}$ from $\mathrm{P}$ to $\mathrm{Q}$ results in negative $\mathrm{D}^{*}$ contrast using $\mathrm{DS}$ in (D). The subtraction of row II from row I shown in row III results 

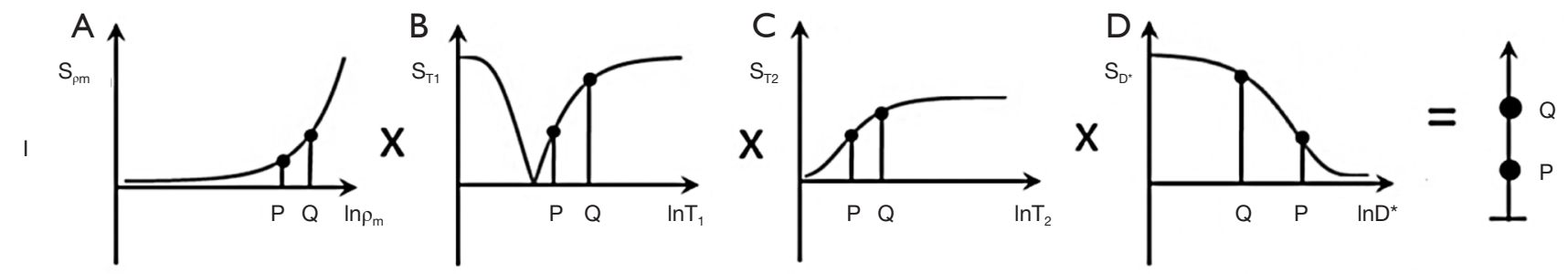

$\|$
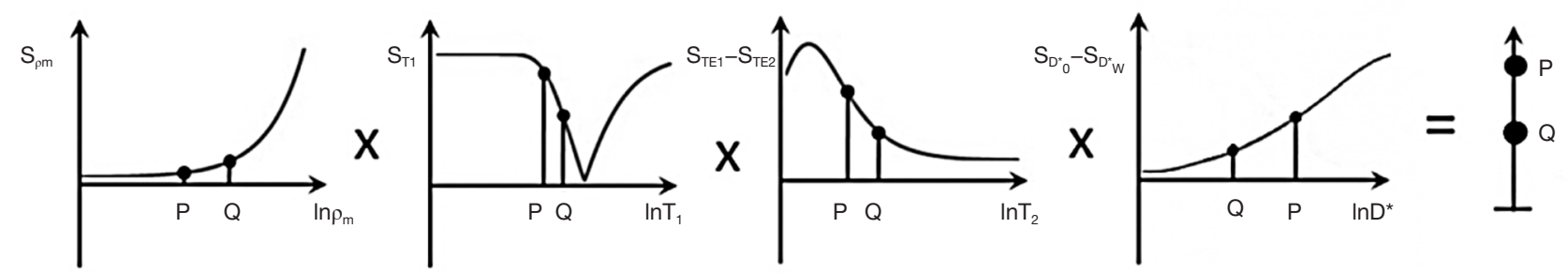

III

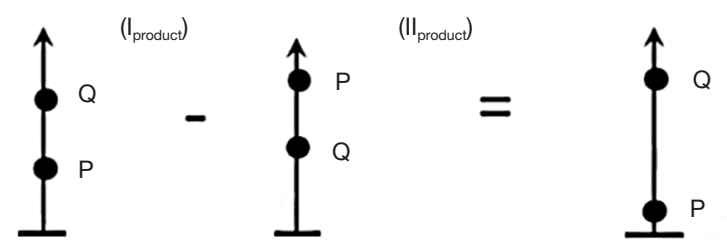

Figure 25 The Subtracted IR Diffusion Echo Subtraction (SIRDES) sequence for an increase in $\rho_{\mathrm{m}}, T_{1}$ and $T_{2}$ with a decrease in $D^{*}$ from $\mathrm{P}$ to Q. The TP-filters for the sequence are shown in the top (I) and middle (II) rows. In the top row, a short $\mathrm{TI}_{\mathrm{s}}$ is used so that increases in $\rho_{\mathrm{m}}, \mathrm{T}_{1}$ and $\mathrm{T}_{2}$ as well as a decrease in $\mathrm{D}^{*}$ from $\mathrm{P}$ to $\mathrm{Q}$ result in positive contrast for each of the four filters, and the product of the signals (far right) shows positive contrast from $\mathrm{P}$ to $\mathrm{Q}$. In the middle row (II), with an intermediate $\mathrm{TI}_{\mathrm{i}}$ an increase in $\mathrm{T}_{1}$ from $\mathrm{P}$ to $\mathrm{Q}$ results in negative contrast. An ES $\mathrm{T}_{2}$-filter is used to produce negative contrast for an increase in $\mathrm{T}_{2}$ from $\mathrm{P}$ to $\mathrm{Q}$. The $\mathrm{D}^{*}$-filter also shows negative contrast for the decrease in $\mathrm{D}^{*}$ from $\mathrm{P}$ to Q. Multiplication (far right) shows negative contrast from $\mathrm{P}$ to $\mathrm{Q}$. In the bottom row (III) subtraction of the product in row II from the product in row I increases the positive contrast from P to Q even further compared to that in row I alone. ES, echo subtraction.

in high positive contrast from $\mathrm{P}$ to $\mathrm{Q}$. This is the situation with tumors and other lesions that show an increase in $\mathrm{T}_{1}$ and $\mathrm{T}_{2}$ with a decrease in $\mathrm{D}^{*}$.

Figure 26 deals with the situation where there is an increase in each of $T_{1}, T_{2}$ and $D^{*}$. In row I, with a short TIs and using $\mathrm{DS}$ in (D), increase in $\mathrm{T}_{1}, \mathrm{~T}_{2}$ and $\mathrm{D}^{*}$ from $\mathrm{P}$ to $\mathrm{Q}$ in (B), (C) and (D) all produce an increase in signal from $\mathrm{P}$ to $\mathrm{Q}$. In row II using $\mathrm{TI}_{\mathrm{i}}$, increases in $\mathrm{T}_{1}$ and $\mathrm{T}_{2}$ (using $\mathrm{ES}$ ) both result in negative contrast. It is not possible to use ES and maintain $\mathrm{D}^{*}$-weighting so no synergistic $\mathrm{D}^{*}$-weighting for tissues is possible in (D). It is only shown with dashed lines. In row III, subtraction of row II from row I leads to positive contrast from $\mathrm{P}$ to $\mathrm{Q}$.

Contrast is relatively straightforward in gray matter, but anisotropy in white matter means that the normal $\mathrm{D}^{*}$ contrast of white matter is highly directional with tracts perpendicular to the diffusion gradient having a lower $\mathrm{D}^{*}$ and higher signal than tracts parallel to the diffusion gradient which have a higher $\mathrm{D}^{*}$. As a result, increase in $\mathrm{D}^{*}$ in disease may be simulated by imaging of a tract more parallel to a gradient than another tract.

Interpretation is easier where all fibers are parallel, e.g., in the corpus callosum in a central sagittal slice, or when symmetry can be used to compare normal and abnormal tracts with the same fiber orientation to the gradients. Use of a diffusion trace averages some of the normal anisotropy. $\mathrm{D}^{*}$-weighted images can be used with tractography to assess gradient directions but mixed/crossing fiber tracts can still be a problem. Other techniques have been implemented for disentangling the effects of normal anisotropy on $\mathrm{D}^{*}$ from those due to disease (42).

\section{(IV) Contrast enhancement with the rSIR, rSIRES and rSIRGES sequences (pre and post i.v. GBCA)}

$\mathrm{T}_{1}$ sequence weighting generally increases in the order SGE, $\mathrm{T}_{1}$-weighted SE, IR gradient echo/fast SE and 

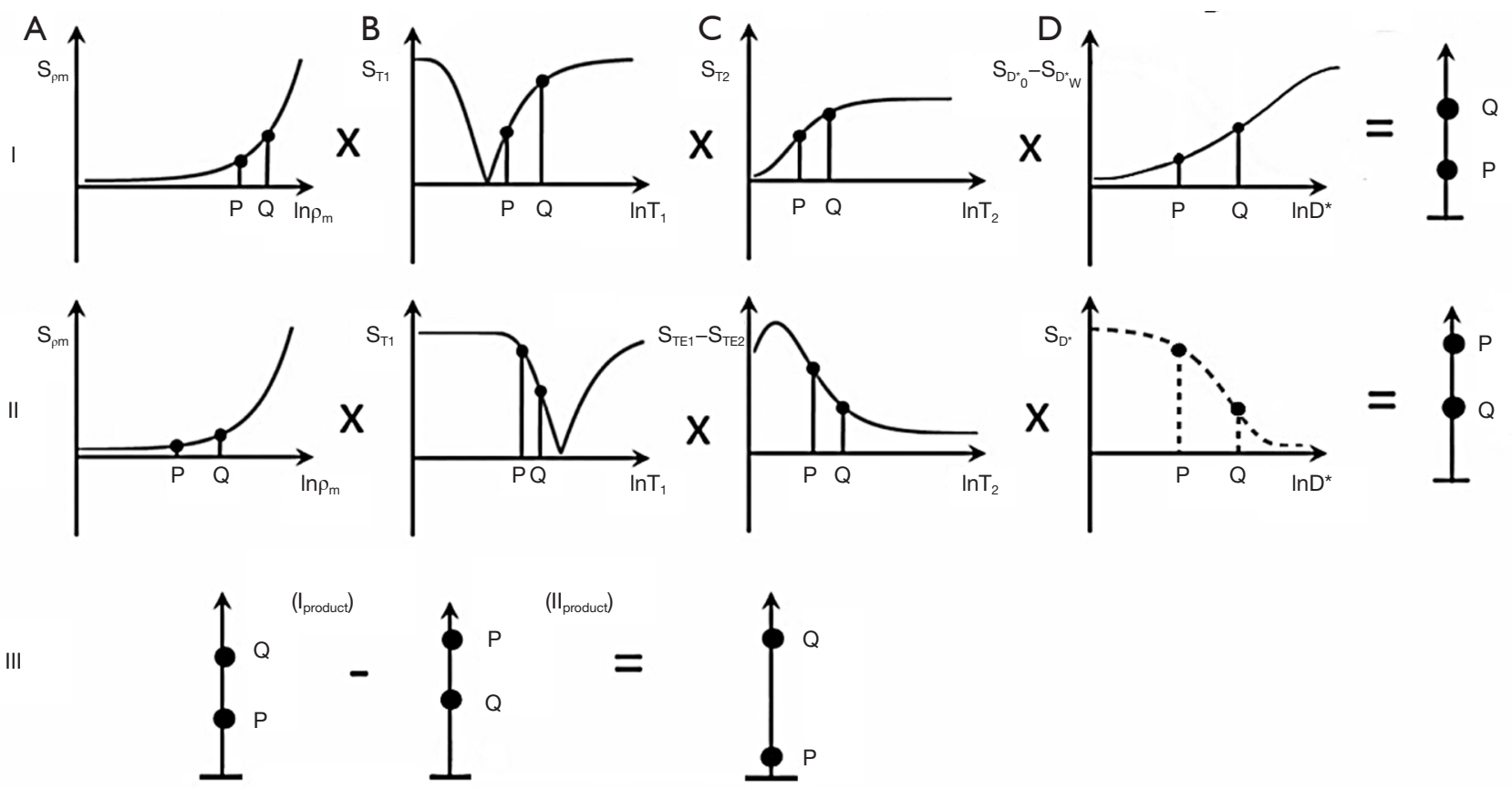

Figure 26 The SIRDES sequence for an increase in each of $\rho_{\mathrm{m}}, \mathrm{T}_{1}, \mathrm{~T}_{2}$ and $\mathrm{D}^{*}$ from $\mathrm{P}$ to $\mathrm{Q}$. In row $\mathrm{I}$ using a short $\mathrm{TI}_{\mathrm{s}} \mathrm{T}_{1}$-filter, conventional $\mathrm{T}_{2}$-filter and DS filter, the increases in $\rho_{\mathrm{m}}, \mathrm{T}_{1}, \mathrm{~T}_{2}$ and $\mathrm{D}^{*}$ result in synergistic positive contrast from $\mathrm{P}$ to $\mathrm{Q}$ shown on the right. In row II, using an intermediate $\mathrm{TI}_{\mathrm{i}} \mathrm{T}_{1}$-filter for $\mathrm{T}_{1}$ and an $\mathrm{ES}$ filter for $\mathrm{T}_{2}$ result in synergistic negative contrast from $\mathrm{P}$ to $\mathrm{Q}$ as shown on the right. It is not possible to retain the negative $\mathrm{D}^{*}$ weighting and reverse the $\mathrm{T}_{2}$ contrast since this would require a heavily diffusion weighted sequence with a short or ultrashort TE. The $\mathrm{D}^{*}$-filter in this row is therefore only shown in dashed line form. In row III, subtraction of the product in row II from the product in row I results in higher positive contrast from $\mathrm{P}$ to $\mathrm{Q}$ than in row I alone. SIRDES, subtracted inversion recovery diffusion and echo subtraction; ES, echo subtraction.

rSIR. The SGE sequence is faster than the rSIR sequence and is therefore better suited to rapid imaging when this is required for high time resolution Dynamic Contrast Enhancement (DCE). In addition, in situations where the GBCA produces a large reduction in $\mathrm{T}_{1}$ a highly $\mathrm{T}_{1}$ sensitive sequence may not be needed and the SGE or $\mathrm{T}_{1}$-weighted $\mathrm{SE}$ sequence may give acceptable clinical results. In MS many lesions (typically chronic or inactive) do not appear to enhance but the number that do show enhancement can be increased both by delayed scanning after i.v. contrast administration, and by the use of double or triple dose GBCAs. In this situation, use of a slower more $\mathrm{T}_{1}$-weighted sequence can be justified, particularly as double or triple dose GBCA administration is probably no longer acceptable because of GBCA retention and toxicity concerns. This applies to MS patients who may require repeat contrast enhanced MRI examinations over an extended period to assess their response to therapy.

Since there is little or no reduction in $\mathrm{T}_{2}$ with conventional doses of GBCAs, an SIR sequence without synergistic $T_{2}$ sequence weighting (as with SIRES) is appropriate. However, if the lesion has an increased T2, contrast enhancement synergistic with this can be achieved with a rSIRES sequence. Diffusion contrast could also be added.

The TIs of the rSIR sequences need to be chosen to accommodate shortening in gray matter and/or white matter and accommodate $T_{1} \mathrm{~s}$ that are shorter than those of normal (or normal appearing) white matter.

With MIOPs there is a reduction in both $\mathrm{T}_{1}$ and $\mathrm{T}_{2}{ }^{*}$ and the reverse SIRGES (rSIRGES) sequence (with a relatively long TE gradient echo) is preferred. This produces a synergistic increase in signal intensity for decreases in $T_{1}$ and $T_{2}{ }^{*}$ (see next section on imaging of iron).

The next four sequences are mainly focused on specificity and are listed in Table 2.

(V) The rSIRGES sequence: imaging of organic iron Organic iron deposited in white matter following 
Table 2 Conventional and MASTIR sequences, mainly for specificity

\begin{tabular}{|c|c|c|c|c|}
\hline Sequence \# & Sequences: conventional; MASTIR & Target tissues/fluids & Common TP changes & Purpose \\
\hline VI & $\begin{array}{l}T_{1} W \text { SE, } T_{2} W \text { SE, } T_{2} \text {-FLAIR; } \\
\text { DESIRE-UTE, STAIRES-UTE }\end{array}$ & White matter & $\begin{array}{l}\downarrow \text { in myelin proton density, } \uparrow \text { in } \\
\text { myelin } T_{1} \text { and } T_{2}\end{array}$ & $\begin{array}{l}\text { Specific imaging of myelin in } \\
\text { white or gray matter }\end{array}$ \\
\hline VIII & rcBV, $T_{2} W$ SE; S IR, S IR & $\begin{array}{l}\text { White, gray matter } \\
\text { or both, or CSF }\end{array}$ & $\begin{array}{l}\uparrow \text { or } \downarrow \text { in } \rho_{\mathrm{m}} \text { of blood, } \pm \text { interstitial } \\
\text { fluid in tissue, CSF }\end{array}$ & Specific imaging of fluids \\
\hline
\end{tabular}

MASTIR, Multiplied, Added, Subtracted and/or FiTted; SE, spin echo; GE, gradient echo; rSIRGES, reverse subtraction IR gradient echo subtraction; MS, multiple sclerosis; FLAIR, FLuid Attenuated inversion recovery; DESIRE, double-echo sliding inversion recovery; UTE, ultrashort echo time; STAIRES, short TR adiabatic pulse prepared inversion recovery echo subtraction; AIR, added inversion recovery; AIRES, AIR echo subtraction; MWF, myelin water fraction; VISTA, VIsualization of Short Transverse relAxation tissue component; CSF, cerebrospinal fluid.

breakdown of myelin in the form of rings, or in gray matter in MS typically reduces both $\mathrm{T}_{1}$ and $\mathrm{T}_{2}{ }^{*}$. Sensitivity to susceptibility effects can be increased by using a long $\mathrm{TE}$ gradient echo acquisition and this can be synergistically combined with a SIR $\mathrm{T}_{1}$-filter in the form of the rSIRGES sequence. It can be targeted on white matter or gray matter and the shortest TI needs to be chosen so that it is significantly shorter than the TI for nulling normal white matter.

With extremely high levels of iron a UTE data acquisition (see next section) may be necessary for signal detection. This applies to MIOPs as well.

\section{(VI) Direct myelin imaging with DESIRE and STAIRES (UTE dcs)}

It is possible to directly image myelin which has an UTE of $0.2-0.3 \mathrm{~ms}$ using an UTE dc (nominal TE $\approx 8-32 \mu \mathrm{s}$ ) coupled with an adiabatic inversion pulse preparation to invert and null the $M_{z}$ of long $T_{2}$ components, and complement this with ES to reduce the signal from tissues that have not been nulled. The DESIRE sequence uses a sliding window to obtain many IR images with TIs covering a wide range of values. Particular TIs can then be chosen to null long $T_{2}$ white matter signals irrespective of their normal $\mathrm{T}_{1} \mathrm{~s}$ or changes in $\mathrm{T}_{1}$ in disease (43).

The STAIR sequence uses a short TR with a single optimized $\mathrm{TI}$ and effectively nulls long $\mathrm{T}_{2}$ signals from tissues or fluids with a wide range of $T_{1} \mathrm{~s}$ (44). It produces efficient nulling but may need to be supported with ES as the STAIRES sequence because the TR cannot be shortened sufficiently to achieve complete signal suppression over a full range of $T_{1}$ s because of specific absorption rate (SAR) concerns (45). Myelin maps can be produced with these sequences and when calibrated can be used to measure myelin proton fraction (MPFs).

\section{(VII) Myelin water AIR (ps'TIs $\left.s_{\mathrm{ij} /}+\mathrm{m}^{\prime} \mathrm{TIs}_{\mathrm{i} / \mathrm{I}}\right)$, AIR echo subtraction (AIRES) and SAIR}

Myelin water has a short $\mathrm{T}_{1}(\sim 400 \mathrm{~ms})$ and short $\mathrm{T}_{2} / \mathrm{T}_{2}{ }^{*}$ ( 10-20 ms). These are both longer than the $T_{1}$ and $T_{2}{ }^{*}$ for myelin $\left(\mathrm{T}_{1}=\sim 300 \mathrm{~ms}\right)$ and $\mathrm{T}_{2}(\sim 0.2-0.3 \mathrm{~ms})$. Extra-axonal, extracellular and intracellular water have a $\mathrm{T}_{1} \mathrm{~s}$ of about 800 $\mathrm{ms}$ and $\mathrm{T}_{2} \mathrm{~s}$ of $60 \mathrm{~ms}(30)$.

It is possible to use an AIR sequence to detect short $T_{1} s$ and so eliminate longer $\mathrm{T}_{1}$ s (Figure 27) and then use a short (but not ultrashort) TE of $\sim 2 \mathrm{~ms}$ to selectively detect myelin water and not myelin. ES can be used to further suppress long $T_{1}$, long $T_{2}$ water as the AIRES sequence. Calibration of the TI can be assisted by performing a DESIRE sequence to provide images with a wide range of TIs.

The SAIR sequence uses subtraction of a longer TI AIR sequence from a shorter TI one to selectively image tissues with a particular range of $T_{1}$ s.

The common approaches to myelin water imaging use multicomponent $T_{2}$ imaging to determine the MWF (31). VISTA (VIsualization of Short Transverse relAxation tissue component) with with long $T_{1}$ signal suppression with two inversion pulses and a partial SSFP with multiple flip angles is another technique that is used (30). 

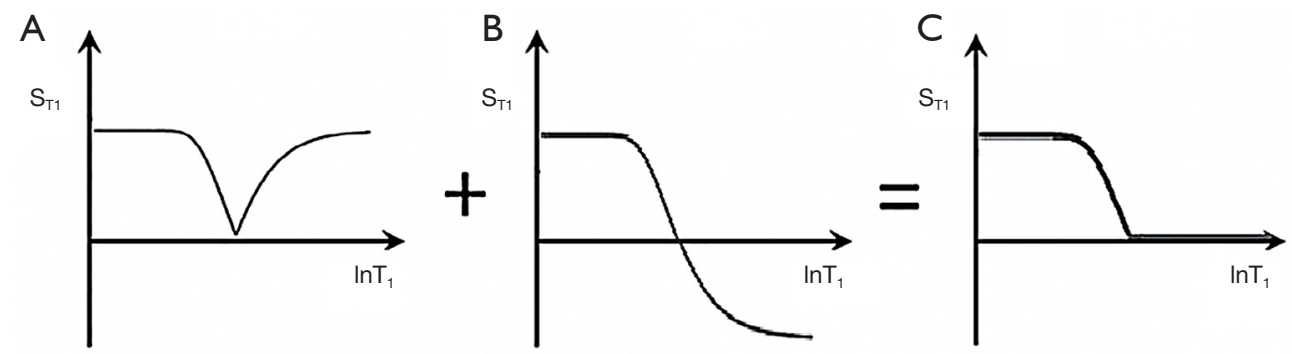

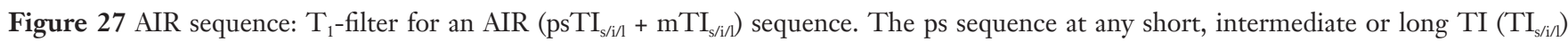
(A) is added to the $m$ reconstructed version of the same sequence with the same TI to give a low pass $T_{1}$-filter in (C). The $S_{T 1}$ values are normalized in this filter. In (C) signal is seen from shorter values of $T_{1}$, there is then a transition band as $T_{1}$ increases, and no signal is seen for longer values of $T_{1}$. AIR, added inversion recovery; $T I$, inversion time; ps, phase sensitive.

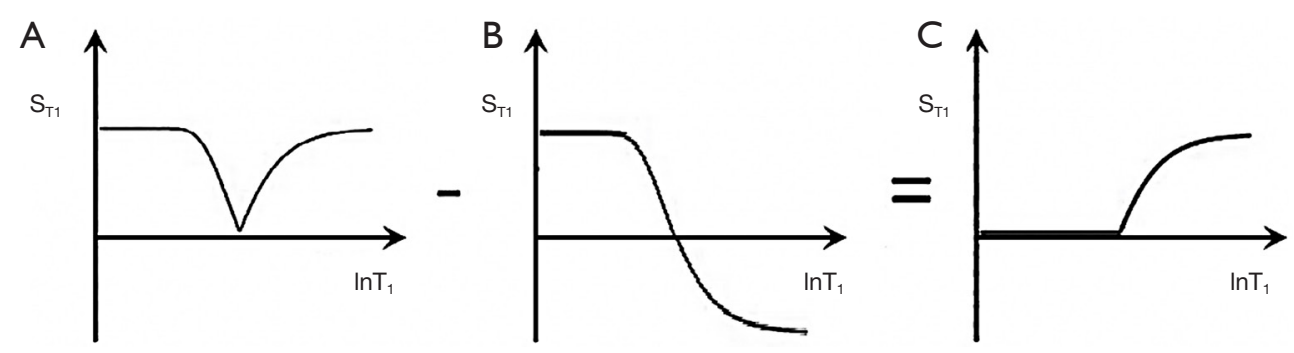

Figure $28 \mathrm{~T}_{1}$-filter for subtracted inversion recovery (SIR) $\left(\mathrm{mTI}_{\mathrm{s} / \mathrm{i} / 1}-\mathrm{psTI}_{\mathrm{s} / \mathrm{i} / \mathrm{l}}\right)$ sequence. The $\mathrm{m}$ sequence (A) at any short, intermediate or long $\mathrm{TI}\left(\mathrm{TI}_{s}, \mathrm{TI}_{\mathrm{i}}, \mathrm{TI}_{1}\right)$ has subtracted from it the corresponding ps sequence with the same TI. This results in a (normalized) high pass $T_{1}$-filter $(C)$. With this filter short $T_{1}$ values are zero. There is a transition band as $T_{1}$ increases and higher signals are seen as $T_{1}$ increases further. TI, inversion time; m, magnitude; ps, phase sensitive.
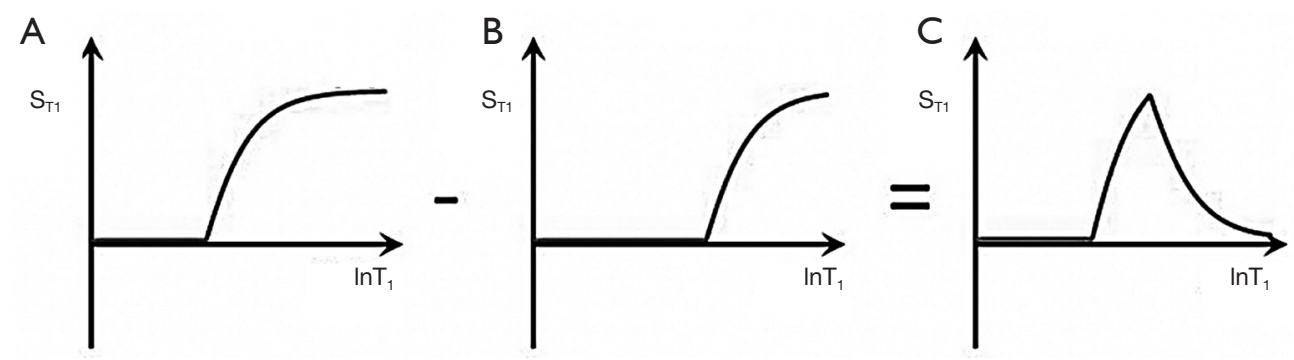

Figure 29 Subtracted SIR $\left[\mathrm{S}^{2} \mathrm{IR}\left(\mathrm{mTI}_{\mathrm{s} / \mathrm{i}}-\mathrm{ps}_{\mathrm{T} / \mathrm{i}}\right)-\left(\mathrm{mTI}_{1}-\mathrm{psTI}_{1}\right)\right]$ sequence. The high pass filter in $(\mathrm{B})$ with a long $\mathrm{TI}_{1}$ produced by subtracting a ps image from an $\mathrm{m}$ image, is subtracted from the high pass filter in (A) which has a short or intermediate $\mathrm{TI}\left(\mathrm{TI}_{\mathrm{s}}\right.$ or $\left.\mathrm{TI}_{\mathrm{i}}\right)$. This gives the band pass filter shown in (C). With longer values of TI this could be used to selectively image blood. SIR, subtracted inversion recovery; TI, inversion time; $\mathrm{m}$, magnitude; $\mathrm{ps}$, phase sensitive.

\section{(VIII) Fluids with $S^{1} I R\left(m T I s_{/ i 1}-p s T I s_{/ i 1}\right)$ and subtracted $S^{1}$ IR $\left(S^{2} I R\right)$}

It is possible to use SIR ( $S^{1} \mathrm{IR}$ sequences with the same TI) to selectively image CSF as well as $\mathrm{S}^{2} \mathrm{IR}$ (subtracted $\mathrm{S}^{1} \mathrm{IR}$ sequences with different TIs) to image blood within the brain (Figures 28,29).

In addition to the MASTIR sequences it is useful to calculate two-point $\mathrm{T}_{2} / \mathrm{T}_{2}{ }^{*}$ and $\mathrm{D}^{*}$ maps to provide checks on the sign of the change in these TPs as well as their magnitude. $\mathrm{T}_{1}$ maps can also be calculated from IR 
sequences with two or more different TIs.

\section{The prostate}

The prostate contains a fibroglandular tissue and is surrounded by a fibrous capsule. It also contains long $\mathrm{T}_{1}$, long $T_{2}$ luminal water $(46,47)$. There is no fat within the gland but there is fat in extraprostate tissue.

Cancer of the prostate in the peripheral and transitional zones shows a quite distinct pattern of reduction in $\mathrm{T}_{1}, \mathrm{~T}_{2}$ and $D^{*}(48,49)$ in contradistinction to tumors elsewhere in the body which usually show an increase in $T_{1}, T_{2}$ with an increase or decrease in $\mathrm{D}^{*}$.

Hemorrhage may be seen in the gland following biopsy with hemorrhage sparing not infrequently seen in higher grade malignancy (50).

The conventional approach is with multiparametric MRI (mpMRI) consisting of three sequences: a $\mathrm{T}_{2}$-weighted SE sequence, a PGSE sequence and a $\mathrm{T}_{1}$-weighted SGE or SE sequence performed before and after i.v. contrast enhancement with a GBCA. This follows Prostate ImagingReporting And Data System (PI-RADS) guidelines (51). biparametric MRI (bpMRI) which uses just the $\mathrm{T}_{2}$ weighted SE sequence and the PGSE sequence, without the contrast enhancement of mpMRI, is also used. There is controversy about whether bpMRI performs as well as mpMRI or not (52).

In comparison with mpMRI, MASTIR sequences offer:

(i) A more highly $T_{1}$-weighted synergistic contrast SIR sequence.

(ii) A SIRES sequence with synergistic contrast for the $\mathrm{T}_{1}$-weighting and the $\mathrm{T}_{2}$-weighting. The conventional $\mathrm{T}_{2}$-weighted sequence is not sensitive to the reduction in $T_{1}$ seen in tumors.

(iii) A SIRDES sequence with synergistic $T_{1}, T_{2}$ and $D^{*}$ contrast. For the reductions in $\mathrm{T}_{2}$ and $\mathrm{D}^{*}$ usually seen in prostate tumors, the conventional PGSE sequence has opposed $\mathrm{T}_{2}$ and $\mathrm{D}^{*}$-weighting and therefore lowers lesion contrast. This may mean that the calculated $\mathrm{D}^{*}$ map which is derived from the PGSE sequence is more useful in diagnosis than the PGSE diffusion weighted image itself. Using the SIRDES sequence shown in Figure 25, reductions in $\mathrm{T}_{1}, \mathrm{~T}_{2}$ and $\mathrm{D}^{*}$ from $\mathrm{Q}$ to $\mathrm{P}$ in the upper row (i) produce synergistic negative contrast with signal from $\mathrm{P}$ lower than that from Q. The middle row (ii) produces synergistic higher positive contrast for decreases in $T_{1}$ and $T_{2}$ from $Q$ to $P$. Subtraction of row II from row I as shown in row III leads to $\mathrm{P}$ even lower signal than Q relative to row I.

(iv) Contrast enhancement with rSIR which has higher $\mathrm{T}_{1}$ sequence weighting than the SGE sequence.

In terms of specificity:

(i) The rSIRGES sequences combines synergistically $\mathrm{T}_{1}$ and $\mathrm{T}_{2}{ }^{*}$ weighting for detection of organic iron effects associated with hemorrhage.

(ii) Ultrashort $T_{2}$ components may be detected.

(iii) Short $T_{2}$ imaging may selectively show fibrotic tissue.

(iv) Luminal water may be detected with $S^{2} I R$ sequence.

Results with mpMRI using PI-RADS were recently reported from a multicenter trial involving members of the Society of Abdominal Radiology Prostate Cancer Diseasefocused Panel (53). The positive predictive value across all 26 centers of mpMRI PI-RADS for higher grade clinically significant (cs) cancers was $49 \%$ and for all cs cancers $35 \%$. The authors stated in the conclusion to their paper "the positive predictive value of the PI-RADS was low and varied widely across centers. We hope these results will motivate discussion and will lead to further research, educational initiatives, quality assurance efforts and, perhaps, PI-RADS updates that may address this issue."

\section{Articular cartilage}

Articular cartilage has particular features of interest from a protocol point of view (54). The normal values of $T_{2}$ cover a very wide range (1-40 ms). In addition, these values are subject to magic angle effects which can see a $2-5$-fold increase in the normal $T_{2}$ as the orientation of fibers to $B_{O}$ (static magnetic field) is changed from $0^{\circ}$ to $55^{\circ}$. This results in considerable overlap between normal and abnormal values of $\mathrm{T}_{2}$. There is also marked anisotropy in $\mathrm{D}^{*}$ values.

Articular cartilage is avascular so contrast enhancement reflects transport of GBCAs from vascularized subchondral bone and joint fluid. The distribution of ionic GBCAs reflects glycosaminoglycan (GAG) concentration as exploited in delayed gadolinium-enhanced MRI of cartilage (dGEMRIC) (55).

Signal from the deep radial and calcified layers is only detectable using UTE or other ultrashort $\mathrm{T}_{2}$ techniques.

Disease may result in complete loss of cartilage. Degeneration is associated with an increase in $T_{1}, T_{2}$ and 
$\mathrm{D}^{*}$ and reduction of magic angle effect.

The $T_{1} s$ of articular cartilage cover a much smaller range of values than the $T_{2} s$ and are not subject to magic angle effects.

Options to take advantage of synergistic contrast in imaging articular cartilage include:

(i) SIR with UTE dc to sensitize the sequences to changes in $T_{1}$ in disease where there is much less normal variation than with $T_{2}$, and there is no confounding magic angle effect.

(ii) SIRES to synergistically use $T_{1}$ and $T_{2}$ contrast to see larger changes in disease which may be more separable from normal variation in $T_{2}$ and magic angle effects.

(iii) Contrast enhancement with rSIR rather than conventional IR sequence as part of a dGEMRIC protocol to increase sensitivity to the presence of GBCAs.

(iv) DESIRE, STAIR and STAIRES to selectively display ultrashort $\mathrm{T}_{2}$ components in the deep radial zone, zone of calcified cartilage and osteochondral junction.

\section{Liver}

Diseases of importance in the liver include tumors and nodules which are covered by Liver Imaging-Reporting And Data System (LI-RADS). These generally display an increase in $T_{1}$ and $T_{2}$ with a decrease in $D^{*}$. Fibrosis follows a similar pattern. Inflammation is associated with an increase in $\mathrm{T}_{1}, \mathrm{~T}_{2}$ and $\mathrm{D}^{*}$. The liver may also accumulate fat and iron, and there are also diseases of the biliary system.

In comparison with conventional protocols the SIR sequence has higher $T_{1}$ sequence weighting than SGE or $\mathrm{T}_{1}$-weighted $\mathrm{SE}$ sequences.

The SIRES sequence adds synergistic $T_{1}$-weighting to the conventional $\mathrm{T}_{2}$-weighted $\mathrm{SE}$ sequence.

Likewise, the SIRDES sequences adds synergistic $\mathrm{T}_{1-}$ weighting and $\mathrm{T}_{2}$-weighting to the conventional PGSE sequence.

The rSIR sequence has higher $T_{1}$ sequence weighting than SGE sequences but has lower time resolution. This may not matter for some applications such as detecting delayed contrast enhancement produced by intra- and extracellular hepato-biliary contrast agents.

The iron sensitivity is higher with the SIRDES sequence than with the SGE sequence.
Ultrashort $\mathrm{T}_{2}$ components can be detected with DESIRE or STAIRES. These have been demonstrated in the liver (56) but their origin and clinical significance are yet to be established.

It is possible to selectively detect bile using $S^{1} \mathrm{IR}$ or $\mathrm{S}^{2} \mathrm{IR}$ sequences exploiting its very long $T_{1}$ rather than its very long $\mathrm{T}_{2}$ as with conventional MR cholangiopancreatography.

With conventional quantitative techniques, proton density fat fraction can be measured with IDEAL, and fibrosis can be assessed as a result of its increased $T_{1}$ after correction for iron $\mathrm{T}_{1}$ shortening effects (35). Fibrosis can also be detected with MR elastography, and iron can be quantified through measurement of $\mathrm{T}_{2}{ }^{*}(57)$.

\section{Bone marrow}

Red bone marrow is an iron rich hematological tissue with a variable $(\sim 50 \%)$ fat content. It also contains blood and is situated within trabecular bone which creates susceptibility effects.

The diseases of principal interest are primary and secondary tumors. Other conditions include increased iron and myelofibrosis with replacement of hematological tissue by fibrosis.

Protocols for detection of the primary tumors MYelomaResponse Assessment and Diagnosis System (MY-RADS) as well as metastatic prostate cancer MEtastasis-Reporting and Data System (ME-RADS) have been established $(58,59)$.

Red bone marrow is notable for the fact that saturation or nulling of the fat component within it alone creates significant contrast for primary and secondary tumors which do not contain fat by reducing the signal from adjacent or surrounding normal marrow and therefore highlighting abnormal tissue. The $\rho_{\mathrm{m}}$ contrast created by fat saturation also applies to PGSE sequences which are routinely fat-saturated. This is to avoid artefacts with EPI imaging and to avoid high signal from fat which has a very low $\mathrm{D}^{*}$. Incomplete fat suppression due to local susceptibility effects and $\mathrm{B}_{\mathrm{O}}$ inhomogeneity may result in spuriously low $\mathrm{D}^{*}$ values for normal red bone marrow hematopoietic tissue using chemical shift-based techniques.

Trabecular bone creates susceptibility effects and makes chemical shift-based techniques for fat signal suppression more difficult. It can be directly imaged (60).

Both primary and secondary tumors display an increase in $T_{1}$ and $T_{2}$ with a decrease in $\mathrm{D}^{*}$. There are major differences between osteolytic and sclerotic lesions, as well 
as between osteoporotic and malignant fractures.

With the STIR sequence $\rho_{\mathrm{m}}, \mathrm{T}_{1}$ and $\mathrm{T}_{2}$ contrast is usually synergistic but blood within the marrow is high signal and potentially reduces lesion contrast. Diffusion weighing provides synergistic $\rho_{\mathrm{m}}, T_{2}$ and $\mathrm{D}^{*}$ contrast. In addition, the diffusion weighting reduces the signal from blood which has a high $\mathrm{D}^{*}$.

\section{MASTIR sequences offer several possibilities}

(i) The SIR sequence reduces fat and fluid signals and produces synergistic $T_{1}$ contrast relative to hemopoietic tissue. The fat saturated $\mathrm{T}_{1}$-weighted $\mathrm{SE}$ sequence also has low signal from fat and blood but has much less $\mathrm{T}_{1}$-weighting.

(ii) The SIRES sequence combines synergistic $T_{1}$ contrast and the $T_{2}$ contrast of conventional $T_{2}$-weighted $S E$ sequences.

(iii) The SIRDES sequence adds synergistic $T_{1}$ contrast to the conventional PGSE sequence.

(iv) rSIR provides higher sensitivity to contrast enhancement than the $\mathrm{T}_{1}$-weighted $\mathrm{SE}$ sequence.

(v) Iron effects can be detected with UTE based techniques.

(vi) UTE DESIRE can detect ultrashort $\mathrm{T}_{2}$ components. The STAIR sequence can be used to detect trabecular bone. It may also be of value in detecting iron effects.

(vii) Blood may be selectively detected with $S^{1} I R$ sequences.

In yellow bone marrow, the fat content is of the order of $95 \%$ with water the remainder. The fat signal can be suppressed with STIR sequences or chemical shift-based fat suppression although susceptibility effects and $B_{O}$ inhomogeneity may make this less effective. Fat signal suppression alone may be sufficient to create contrast for water containing lesions including metastases and bone marrow edema. In edema T1-weighted out-of-phase images of yellow bone marrow with cancellation of signal between water and fat can be synergistic with reduction in signal from an increase in $\mathrm{T} 1$ because of the water present in the edema.

\section{Development}

This encompasses:

(i) Use of other TPs including MT, other $\mathrm{T}_{2}$ preparations, perfusion and blood flow for angiography as well as more advanced diffusion and susceptibility.

(ii) Use of other pulse sequences including more advanced forms of fMRI in the brain and body.

(iii) Quantitation for example quantitative forms of DESIRE to assess myelin.

(iv) Improvement of scan time with SENSE and compressed SENSE as for DESIRE (43).

(v) Clinical research designed to exploit the increased sensitivity of MASTIR sequences such as MS, Alzheimer's disease, traumatic brain injury and tumors as well as the specificity of DESIRE and STAIRES for myelin.

\section{Discussion}

The approach used in this paper is based on understanding the signal, contrast and weighting of MR images using TPfilters. This has been applied to MASTIR sequences to provide options to develop synergistic contrast. Approaches have been organized in the form of protocols for MR examinations of the CNS, body and musculoskeletal system with the objective of improving on the performance of the sequences normally used in these protocols.

\section{TPs}

The primary focus is on $\rho_{\mathrm{m}}, \mathrm{T}_{1}, \mathrm{~T}_{2}$ and $\mathrm{D}^{*}$ but there are also important applications of susceptibility, chemical shift, ultrashort $\mathrm{T}_{2}$ tissues, MT and contrast agents.

In disease there are often concurrent changes in $\mathrm{T}_{1}, \mathrm{~T}_{2}$ and $\mathrm{D}^{*}$ and a strategy to improve sensitivity is to synergistically use changes in two or more major determinants of contrast such as $T_{1}, T_{2}$, and $D^{*}$. This is dependent on knowing whether the change in disease is an increase or decrease in the TP.

Generally, $T_{1}$ and $T_{2}$ are increased in disease (except where paramagnetics such as iron or contrast agents are involved and they are usually decreased but $\mathrm{D}^{*}$ is more variable with increases in many acute and chronic diseases, but reduction in many tumors, acute infarction, acute infections, and some other conditions. Prostate cancers are unusual in showing a reduction in each of $\mathrm{T}_{1}, \mathrm{~T}_{2}$ and $\mathrm{D}^{*}$.

\section{MASTIR sequences}

The concept here is a group of sequences rather than a single sequence, and employs multiplication, subtraction, addition and/or fitting of different components of the IR sequence. Contrast produced by each of $T_{1}, T_{2}$ and $D^{*}$ is reversible within sequence segments. The overall contrast 
for subtracted sequences depends on the order of the subtraction.

A difficulty with the SIR sequence is the increased time required for two IR sequences. This can be reduced with SENSE and compressed SENSE but it is difficult to achieve the higher time resolution achievable with SGE sequences in DCE where the contrast agent leads to large changes in $\mathrm{T}_{1}$ and the sequence does not require high sensitivity.

\section{Implementation}

In principle, little technical effort is required to implement many of the common MASTIR sequences although in practice problems may arise. The basic SIR sequence simply requires subtraction of two registered IR images with different TIs and short or UTEs. The SIRES sequences use the same formula with long TEs and the subtraction of a longer TE image from a shorter one. Suitable acquisitions for MASTIR sequences are available on most clinical MRI systems.

The DESIRE sequence is more technically demanding but has considerable value in providing IR images with a wide range of TIs to establish nulling points for normal and abnormal tissues and fluids. The STAIR and STAIRES sequences are easier to implement than DESIRE and provide nulling of long $T_{2}$ components over a wide range of $T_{1}$ values to allow imaging of ultrashort $T_{2}$ species such as cortical and trabecular bone as well as myelin.

\section{Clinical set up}

To establish the $T_{1}$-filter for SIR based sequences requires:

(i) A knowledge of the initial $\mathrm{T}_{1}$ to set up the first TI.

(ii) A knowledge of the sign of the change in $T_{1}$ expected, i.e., increase/decrease or both.

(iii) A knowledge of the range of change in $T_{1}$ so that the second TI can be chosen to accommodate the expected change(s) in signal.

To set up $\mathrm{T}_{2}$-filters requires:

(i) A knowledge of the sign of the change in $T_{2}$.

(ii) The value of $T_{2}$ so that an appropriate $T E$ can be chosen using $\mathrm{TE}=\mathrm{T}_{2}$ for maximizing $\mathrm{C}_{\mathrm{ab}}$ with a $\ln \mathrm{X}$ scale as a starting point, but noting that $\mathrm{Cfr}$ has no corresponding maximum value.

To set up $\mathrm{D}^{*}$-filters requires:

(i) A knowledge of the equivalent image in $\mathrm{D}^{*}$ (i.e., increase or decrease), and

(ii) General maximization of sequence weighting for $\mathrm{C}_{\mathrm{ab}}$ using a $\ln \mathrm{X}$ scale for which $\mathrm{b}=1 / \mathrm{D}^{*}$ as a starting point, but noting that $\mathrm{Cfr}$ has no maximum corresponding value.

\section{Image interpretation}

This may initially be disconcerting with, for example, a very heavily $\mathrm{T}_{1}$-weighted SIR image of the brain appearing like a heavily $\mathrm{T}_{2}$-weighted $\mathrm{SE}$ image.

CSF and other fluids which are often at the top of the display range because of their very long $T_{1} S$ and $T_{2} s$ may be in the center of the display range with SIR images. Another issue is the quite different appearances of normal images resulting from reversing the order of subtraction of the SIR group of images. Contrast enhancement with GBCAs increases the signal with rSIR sequences and this may be in normal tissue, e.g., grey matter in the brain and look as though it is a SE $\mathrm{T}_{2}$-weighted image showing contrast enhancement. This is contrary to conventional clinical experience.

It is possible to follow the acquired images and so observe the effects of additional subtractions and so recognize a discordant effect.

It is also possible to run conventional sequences in parallel and extrapolate from these to understand the contrast seen on MASTIR images taken of the same slice during the same examination.

The wide range of $\mathrm{D}^{*}$ values in white matter due to anisotropy results in overlap between normal and abnormal values of $\mathrm{D}^{*}$. Work is being done on techniques to disentangle the two effects and obtain values of fractional anisotropy which are independent of fiber orientation dispersion (42).

\section{Existing synergistic contrast sequences}

(i) The STIR sequence can have its $\mathrm{T}_{1}$-weighting increased by operating it as a SIRES sequence.

(ii) The DIR sequence can also be converted to a SIRES sequence. This means that the $T_{1}$-weighting of the second SIR sequence is synergistic within the chosen range of $T_{1}$ values, rather than slightly opposed as it is for white matter suppressed DIR images of the brain.

(iii) The PGSE sequence can have:

* Added to it high $\mathrm{T}_{1}$-weighting with the SIRES sequence;

* Synergy of $\mathrm{D}^{*}$ and $\mathrm{T}_{2}$, not opposition as in, for example, increased $\mathrm{D}^{*}$ lesions. 


\section{(iv) MP2RAGE.}

The MP2RAGE sequence multiplies a short TI segment (e.g., $800 \mathrm{~ms}$ at 3T) by a longer one (e.g., 2,200 ms at 3T) and uses ps reconstruction (33). The signals are normalized and this reduces or eliminates contributions to contrast from $\rho_{\mathrm{m}}$ and $T_{2}$. It is univariate and is described by a $T_{1}$ filter. The sequence can be used to directly measure $T_{1}$. It is insensitive to B1 and receiver field inhomogeneities and is optimized to maximize contrast between white matter, gray matter and CSF. White matter is higher signal than gray matter and the sequence shows higher $T_{1}$ contrast than MP-RAGE. The slopes of the two $T_{1}$ TI filters are both negative and change in $T_{1}$ is used twice to create synergistic contrast from a single TP.

Abnormalities in MS appear of lower signal and the sequence can be more sensitive in this disease than conventional sequences used in standard protocols (61).

In comparison with the SIR sequence, the MP2RAGE sequence uses multiplication of two quite different TI segments, rather than subtraction of two fairly close together TI segments with the SIR sequence. The MP2RAGE sequence is not reversible.

In comparison with the DIR sequence, the MP2RAGE sequence has higher $T_{1}$ weighting because the positive $T_{1}$ weighting of the shorter TI segment of the DIR sequence is slightly opposed by the negative $T_{1}$-weighting of the longer TI segment for white and gray matter. The DIR sequence shows synergistic contrast from the $T_{1}$ and $T_{2}$ segments for increases in $T_{1}$ and $T_{2}$ in lesions, not from double use of $\mathrm{T}_{1}$-weighting as with the MP2RAGE sequence.

\section{Comparison between scMRI and mpMRI}

The term mpMRI is employed generally as in the CNS to mean use of a variety of different sequences with dependence on different TPs, e.g., $\mathrm{T}_{1}, \mathrm{~T}_{2}, \mathrm{~T}_{2}{ }^{*}, \mathrm{D}^{*}$, susceptibility etc. It is also used more specifically in the body to mean three specific sequences, e.g., for the prostate tumors (i) a $\mathrm{T}_{2}$-weighted SE, (ii) a PGSE sequence, and (iii) contrast enhancement with a SGE or SE sequence. In these examinations each sequence is usually optimized as far as possible to show one TP, e.g., $T_{1}$ or $T_{2}$ although this is not possible with clinical PGSE sequences which are inherently positively $\mathrm{T}_{2}$ - and negatively $\mathrm{D}^{*}$-weighted.

With scMRI employed for sensitivity purposes, synergistic contrast is used in the SIR sequence to increase the $T_{1}$-weighting, synergistic $T_{1}$ and $T_{2}$ contrast is used with the SIRES sequence to provide synergistic $T_{1}$ and $T_{2-}$ weighting, and synergistic $T_{1}, T_{2}$ and $D^{*}$-weighting is used with the SIRDES sequence.

For specificity a SIRGES sequence may be used to detect decreases in $T_{1}, T_{2}{ }^{*}$ from iron, and DESIRE or STAIRES can be used to detect ultrashort species such as myelin and cortical bone. AIR or AIRES sequences can detect short $T_{2}$ species such as tendons and ligaments and $\mathrm{S}^{1} \mathrm{IR} / \mathrm{S}^{2} \mathrm{IR}$ can be used for fluids.

mpMRI employs single use single TP sequences for $T_{2}-$ and $\mathrm{T}_{1}$-weighting for contrast enhancement without synergy. The PGSE sequence has the potential for synergistic contrast and this is realized when $T_{2}$ is increased and $\mathrm{D}^{*}$ is reduced as in many body tumors. However, in prostate tumors both $\mathrm{T}_{2}$ and $\mathrm{D}^{*}$ are decreased and their contrast is opposed.

\section{Comparison between scMRI and fMRI}

The term fMRI is used to describe the activation experiment in which signal from the brain is increased during brain activity when using a $\mathrm{T}_{2}{ }^{*}$-weighted sequence as a result of an increase in the $\mathrm{T}_{2}{ }^{*}$ in blood within tissue.

fMRI is also used in the body to describe examinations of tumors using diffusion weighting and DCE with or without MR spectroscopy. fMRI is used in cardiac imaging to describe physiological changes in tissue motion and blood flow. It is also used in the musculoskeletal system to describe dynamic changes in position at joints as well as changes in muscles and tendons during physical activity.

In comparison, scMRI is specific for use of TPs and signal suppression to improve image contrast. It is in principle applicable to the basic activation experiment in the brain as well as sequences used for fMRI in the body.

\section{Quantitation}

scMRI is primarily concerned with image contrast and is usually used qualitatively for lesion detection. The origins of this contrast can be described quantitatively using the TP-filters approach. Basic quantitation is used with scMRI to provide a check on the sign of changes in TPs and an estimate of their magnitude. Accurate quantitation of TPs can be performed using high contrast clinically useful sequences but often it requires dedicated pulse sequences as well as modelling techniques, and limits on the time available for clinical acquisitions may mean that it is not practical to also make these pulse sequences of high 
qualitative diagnostic value.

\section{Problems}

(i) The MR system may not have both ps and $m$ reconstructions available, have no IR preparation modules for some sequences, and/or not allow certain sequence parameters (e.g., long TIs).

(ii) The machine may rescale images, process them, and may not allow access to them in unprocessed form.

(iii) The basic sequences, e.g., UTE may be of poor quality or not include inversion pulses.

(iv) MASTIR sequences are signal-to-noise ratio (SNR) and carrier-to-noise ratio (CNR) limited and many involve selective attenuation of signals to create contrast. This may require use of lower spatial resolution acquisitions and less aggressive sequence parameters, e.g., shorter TEs, lower b values and longer $T_{1}$ differences between short and long TIs for subtraction.

(v) Ballpark values of $T_{1}$ from $\left(T_{1}=1.44\right.$ TI nulling), $\mathrm{T}_{2}$ from the dual echo fast spin echo (FSE), $\mathrm{T}_{2}{ }^{*}$ from multi-echo gradient echo and $\mathrm{D}^{*}$ from two diffusion weighted sequences are checks, e.g., for unexpected values of $T_{1}, T_{2} / T_{2}{ }^{*}$. In in vitro work, the $T_{1}, T_{2}, T_{2}^{*}$ and $D^{*}$ of formalin fixed tissue may be much shorter than unfixed and in vivo tissues.

(vi) Checking images at different stages of the subtraction process may be helpful in seeing where the contrast is increased or decreased.

(vii) The sequence may be wrongly targeted, e.g., for an increase in $T_{1}$ in grey matter, not a decrease in $\mathrm{T}_{1}$. Use of $\mathrm{T}_{1} \mathrm{~s}$ to provide a wide range of contrast response (e.g., $\mathrm{T}_{1} \mathrm{~s}$ less than white matter to $\mathrm{T}_{1} \mathrm{~s}$ greater than white matter may be helpful at the expense of lower $T_{1}$ contrast).

(viii) If the signal level is very low there may be no scope to develop contrast. Calculated values, e.g., of $\mathrm{T}_{2}$, $\mathrm{T}_{2}{ }^{*}$ and $\mathrm{D}^{*}$ may also be inaccurate in this situation.

(ix) It is possible to reach the maximum allowable signal value, e.g., with contrast agent shortening in white matter using a rSIRES sequence and no further increase occurs. The enhancement does not "get off the scale".

(x) The contrast may be excessive. MASTIR sequences are often used to provide high sensitivity to small changes in TPs including those due to low levels of contrast enhancement, not to increase further the contrast of lesions that are already obvious using other approaches. This may lead to the high contrast obscuring more subtle features.

\section{Summary}

(i) The first objective of scMRI is to find ways to use a change in TP twice or more to increase contrast.

The second objective is to find ways to use changes in two or more TPs to increase contrast.

The third objective is to supplement the contrast developed using the first two mechanisms by reduction in the high signals of normal tissues so that high signal contrast in lesions becomes more obvious.

These three objectives may be combined.

(ii) Frequently, several TPs are changed in disease and synergistic contrast can be used to exploit each of these changes to increase sequence sensitivity.

(iii) Synergistic contrast can also be used to selectively highlight effects due to particular changes in TPs (e.g., reduction in $\mathrm{T}_{1}$ and $\mathrm{T}_{2}{ }^{*}$ ) or to selectively image tissues as a function of their $\mathrm{T}_{1} \mathrm{~s}, \mathrm{~T}_{2} \mathrm{~s}$, chemical shift and other TPs.

(iv) The first examples of synergistic pulse sequences were the STIR and DIR sequences in forms which both produce synergistic positive contrast for concurrent increases in $T_{1}$ and $T_{2}$ in lesions. They were each both $T_{1}$ - and $T_{2}$-weighted. The sequences also suppressed fat, white or gray matter and/or CSF in head examinations.

(v) The long TE necessary to apply gradients after the $90^{\circ}$ pulse and around the $180^{\circ}$ pulse in the PGSE sequence means that clinical heavily $\mathrm{D}^{*}$-weighted sequences are inherently heavily $\mathrm{T}_{2}$-weighted as well. The $\mathrm{T}_{2}$-weighting is positive and the $\mathrm{D}^{*}$ weighting is negative; the two are opposed. If $\mathrm{T}_{2}$ is increased and $\mathrm{D}^{*}$ is decreased the contrast both TPs produce is positive and synergistic. This is the case in acute cerebral infarction and many tumors.

(vi) Major contrast determinants are $T_{1}, T_{2}$ and $D^{*} . r_{m}$ is usually a minor determinant but may become a major one with MT and the use of fat saturation as in red bone marrow where it can produce a $50 \%$ signal reduction. 
(vii) To achieve synergistic contrast for both positive and negative changes in $T_{1}, T_{2}$ and $D^{*}$ it is necessary to reverse the sign of the sequence weighting of the relevant pulse sequence. This can be done for $T_{1}$ by changing the TI of an IR sequence, for $\mathrm{T}_{2}$ by subtracting a longer TE image from a shorter one (ES) and for $\mathrm{D}^{*}$ subtraction of a high $\mathrm{b}$ value image from a lower one (DS). These changes are of the segments of a sequence.

(viii) It is also possible to reverse the order of subtraction of one sequence from another and this reverses contrast. It is designated by $r$ for reversal.

(ix) The absolute $\mathrm{C}_{\mathrm{ab}}$ contrast produced by a $\mathrm{TP}$ segment of a sequences is given by:

$\mathrm{C}_{\mathrm{ab}}=\partial \mathrm{S}_{\mathrm{TP}} / \partial \mathrm{TP} \times \Delta \mathrm{TP}$

using a linear $\mathrm{X}$ axis. $\partial \mathrm{S} / \partial \mathrm{TP}$ is the first partial derivative with respect to the $\mathrm{TP}$ and $\Delta \mathrm{TP}$ is the change in TP.

Using a naturally $\ln \mathrm{X}$ axis the equation is:

$\mathrm{C}_{\mathrm{ab}}=\partial \mathrm{S}_{\mathrm{TP}} / \partial \ln \mathrm{TP} \times \Delta \mathrm{TP} / \mathrm{TP}$

where $\partial \mathrm{S}_{\mathrm{TP}} / \partial \ln \mathrm{TP}$ is the partial derivative with respect to the natural log of the TP and $\Delta \mathrm{TP} / \mathrm{TP}$ is the fractional change in TP.

(x) The fractional contrast $\mathrm{C}_{\mathrm{fr}}=\Delta \mathrm{S} / \mathrm{S}$ for a single $\mathrm{TP}$ using a logarithmic $\mathrm{X}$ axis is:

$\mathrm{C}_{\mathrm{fr}}=\frac{1}{\mathrm{~S}_{\mathrm{TP}}} \frac{\partial \mathrm{S}_{\mathrm{TP}}}{\partial \ln \mathrm{TP}} \times \frac{\Delta \mathrm{TP}}{\mathrm{TP}}$

For several TPs in a sequence using a logarithmic $\mathrm{X}$ axis:

$\mathrm{C}_{\mathrm{fr}}=\sum_{\mathrm{TP}} \frac{1}{\mathrm{~S}_{\mathrm{TP}}} \frac{\partial \mathrm{S}_{\mathrm{TP}}}{\partial \ln \mathrm{TP}} \times \frac{\Delta \mathrm{TP}}{\mathrm{TP}}$

(xi) From Eq. [17] the overall fractional contrast is the sum of the contributions made by each TP. These contributions are the product of the normalized first partial derivative for the TP multiplied by the fractional change in the TP. If both these quantities are positive, or both are negative, the contribution to contrast is positive. If one is positive and other is negative, the contribution is negative. What is sought with synergistic contrast from several TPs is all positive, or all negative contributions to contrast from all the TPs. (xii) With a single TP multiple use sequence (e.g., $\mathrm{T}_{1}$, $\mathrm{T}_{2}$ ), positive or negative change in the TP produces positive or negative synergistic contrast without complication providing that the chosen range of $\mathrm{T}_{1} \mathrm{~s}$ is appropriate. However, with two different $\mathrm{TPs}$ creating synergistic contrast, the contrast produced by each TP needs to be matched in sign to produce either overall positive or negative contrast. If the contrast for the segments is opposed, the overall contrast is reduced.

(xiii) With the PGSE the sequence weighting is positive for $T_{2}$, but negative for $\mathrm{D}^{*}$. With increase in $\mathrm{T}_{2}$, $\mathrm{D}^{*}$ must be decreased to produce high positive contrast. This occurs in some conditions (acute cerebral infarction, many tumors) but more often $\mathrm{D}^{*}$ is also increased (chronic infarction, demyelination, infection, etc.) and the contrast is opposed. As a result, contrast may be less on the $\mathrm{b}=500-1,000 \mathrm{~s} / \mathrm{mm}^{2}$ image than it is on the $\mathrm{b}=0-20 \mathrm{~s} / \mathrm{mm}^{2}$ image and there is little or no direct benefit from the diffusion weighted image itself. However, the calculated map of the $\mathrm{D}^{*}$ values may show the increase in $\mathrm{D}^{*}$ as a highlighted region and allow the lesion to be identified and the image to be interpreted.

(xiv) Another contrast synergy with the same TP may occur with blood flow when highlighted inflowing blood in one sequence has subtracted from it low signal dephased blood in another sequence.

(xv) Susceptibility weighted imaging when both magnitude and phase data produced by a difference in susceptibility are multiplied together is another example of same TP contrast synergy.

(xvi) MT produces a reduction in both observed $r_{m}$ and observed $T_{1}$ and these changes may be used synergistically to increase or decrease contrast.

(xvii) MASTIR sequences consist of:

I. A MIR group including:

* The DIR sequence typically a long TI segment nulling CSF or blood multiplied by a short TI segment nulling fat, white matter or gray matter.

* The MP2RAGE sequence which also has a long TI and a short TI segment multiplied together. It is normalized which largely eliminates $r_{m}$ and $T_{2}$ effects. It is a dual use single TP $\left(T_{1}\right)$ synergistic negative $T_{1}$-weighted sequence.

II. An AIR group. 
* The AIR sequence adds ps and $m$ reconstructed images which shows shorter $T_{1}$ tissues and cancels to zero longer $T_{1}$ tissues and fluids.

* AIRES. This adds ES to the AIR sequence as an additional filter.

* SAIR. This is a SAIR sequence which shows specific tissues as a function of their $T_{1}$.

III. A SIR group.

This has eight subsections, the first five of which are SIR variants:

* The SIR sequence subtracts an intermediate $\mathrm{TI}_{\mathrm{i}} \mathrm{m}$ reconstructed image for a short TIs $\mathrm{m}$ reconstructed image. It shows high synergistic contrast for $T_{1}$ s between the values at the nulling points for TIs and $\mathrm{TI}_{\mathrm{i}}$. The subtraction can be reversed with the rSIR sequence which shows high increased signal for a reduction in $\mathrm{T}_{1}$ within the same limits.

* The SIRES sequence extends the SIR sequence by adding $\mathrm{T}_{2}$-weighting. In order to align the contrast an ES is necessary to increase the $T_{2}$ contrast in either the first or second acquisition (Figure 24). This sequence can also be reversed (rSIRES) to show high contrast for a reduction in both $T_{1}$ and $T_{2}$.

* The SIRDES sequence adds to the SIRES sequence diffusion weighting. DS is necessary to allow reversal of diffusion contrast. The $T_{1}$, $\mathrm{T}_{2}$ and $\mathrm{D}^{*}$ contrast is synergistic for increases in $\mathrm{T}_{1}$ and $\mathrm{T}_{2}$ with a decrease in $\mathrm{D}^{*}$ (Figure 26). It is also synergistic for increases in all three of $\mathrm{T}_{1}, \mathrm{~T}_{2}$ and $\mathrm{D}^{*}$ (Figure 27) but it is not possible to obtain high $\mathrm{D}^{*}$-weighting with a very short TE so that reversal of the $T_{2}$-weighting is not possible with high $\mathrm{D}^{*}$-weighting.

* The SE segment may be replaced by a GE segment with a long $\mathrm{T}_{2}$ to give $\mathrm{T}_{2}{ }^{*}$-weighting as the SIRGES sequence. This can be reversed as the rSIRGES sequence. Reduction in $\mathrm{T}_{2}{ }^{*}$ and $\mathrm{T}_{1}$ then produces synergistic contrast.

* The SIRDGES sequence uses the SIRDES sequence with a gradient echo.

* The DESIRE sequence uses a sliding window to generate images with a range of TIs. This can be used to select particular TIs to null long $T_{2}$ components with different $T_{1}$. It is typically operated with a UTE dc to selectively image myelin or other ultrashort $T_{2}$ tissues.
The STAIRES sequence uses a short TR and a single TI to null long $T_{2}$ tissues with a wide range of $T_{1}$ s. It is also used with a UTE dc for imaging ultrashort $\mathrm{T}_{2}$ tissues.

* Subtraction of a m from a ps sequence selectively shows fluids as the $\mathrm{S}^{1} \mathrm{IR}$ sequence. Subtraction of this sequence again gives the $S^{2} I R$ sequence which may be used to show a single fluid, e.g., blood.

* The IRES sequence nulls long $T_{2}$ components and then uses ES to show short or ultrashort $\mathrm{T}_{2}$ components. It is suitable for tissues with a single $T_{1}$. The STIRES sequence nulls WAT and leaves longer $T_{1}$ BAT. The fat component in BAT can then be successfully image using a Dixon subtraction, i.e., in-phase image minus out-of-phase image.

IV. The FIR group.

This includes sequences which obtain several TI images. These may be used to measure $T_{1}$ (MPnRAGE and shMOLLI) or to find the optimal TI for nulling long $\mathrm{T}_{2}$ signal (DESIRE).

(xviii) Contrast enhancement with GBCAs produces a shortening of $T_{1}$ that produces positive contrast with rSIR sequences. Contrast enhancement with MIOPs producing a reduction in $\mathrm{T}_{2}{ }^{*}$ and $\mathrm{T}_{1}$. This produces positive contrast with a rSIRGES sequence which has a long TE gradient echo to provide sensitization to the $\mathrm{T}_{2}{ }^{*}$ shortening.

(xix) Protocols are generally organized to:

* Increase sensitivity with multi-use synergy with a single TP as well as multiple TP synergies with two or more TPs.

* Improve specificity when signals from one tissue or fluid, or group of these are highlighted and signals from others are reduced or suppressed.

(xx) Organic iron including hemoglobin breakdown products are paramagnetic and relatively specific in producing a reduction in $T_{1}$ and $T_{2}{ }^{*}$ although other paramagnetics and contrast agents do this as well.

(xxi) Cortical bone, calcified cartilage, myelin and high concentration iron have ultrashort $\mathrm{T}_{2} \mathrm{~S}\left(0.1<\mathrm{T}_{2}<1 \mathrm{~ms}\right)$ which is also relatively specific.

(xxii) Fluids have increased $\mathrm{T}_{1} \mathrm{~s}$ and $\mathrm{T}_{2} \mathrm{~s}$ is the general order: (i) blood, (ii) interstitial fluid, bile and lymph, and (c) urine, CSF.

(xxiii) Clinical protocols employ single use single TP sequences such as the $T_{1}$-weighted, $T_{2}$-weighted 
SE sequences, and contrast enhancement with a $\mathrm{T}_{1}$-weighted sequence. These are not synergistic. PGSE sequences are both positively $T_{2}$ and negatively $\mathrm{D}^{*}$-weighted and are synergistic for an increase in $T_{2}$ and decrease in $\mathrm{D}^{*}$.

(xxiv) Options exist for replacing elements of clinical protocols with MASTIR sequences which may make more effective use of the changes in TPs seen in disease.

\section{Acknowledgments}

Funding: The authors acknowledge grant support from the NIH (RO1 NS092650).

\section{Footnote}

Conflicts of Interest: All authors have completed the ICMJE uniform disclosure form (available at http://dx.doi. org/10.21037/qims-20-795). JD serves as an unpaid editorial board member of Quantitative Imaging in Medicine and Surgery. The other authors have no conflicts of interest to declare.

Open Access Statement: This is an Open Access article distributed in accordance with the Creative Commons Attribution-NonCommercial-NoDerivs 4.0 International License (CC BY-NC-ND 4.0), which permits the noncommercial replication and distribution of the article with the strict proviso that no changes or edits are made and the original work is properly cited (including links to both the formal publication through the relevant DOI and the license). See: https://creativecommons.org/licenses/by-nc-nd/4.0/.

\section{References}

1. Bydder GM, Young IR. Clinical use of the inversion recovery sequence. J Comput Assist Tomogr 1985;9:659-75.

2. Redpath TW, Smith FW. Technical note: use of a double inversion recovery pulse sequence to image selectively gray or white brain matter. Br J Radiol 1994;67:1258-63.

3. Young IR, Szeverenyi NM, Du J, Bydder GM. Pulse sequences as Tissue Property filters (TP-filters): A way of understanding the signal, contrast and weighting of Magnetic Resonance Images. Quant Imaging Med Surg 2020;10:1080-120.

4. Ma YJ, Fan S, Shao H, Du J, Szeverenyi NM, Young IR, Bydder GM. Clinical use of Multiplied, Added, Subtracted and/or fiTtedInversion Recovery (MASTIR) pulse sequences. Quant Imaging Med Surg 2020;10:1334-69.

5. Hawkes RC, Holland GN, Moore WS, Worthington BS. Nuclear magnetic resonance (NR) tomography of the brain: a preliminary clinical assessment with demonstration of pathology. J Comput Assist Tomogr 1980;4:577-86.

6. Young IR, Hall AS, Pallis CA, Legg NJ, Bydder GM, Steiner RE. Nuclear magnetic resonance imaging of the brain in multiple sclerosis. Lancet 1981;2:1063-6.

7. Bailes DR, Young IR, Thomas DJ, Straughan K, Bydder GM, Steiner RE. NMR imaging of the brain using spinecho sequences. Clin Radiol 1982;33:395-414.

8. Bydder GM, Steiner RE, Young IR, Hall AS, Thomas DJ, Marshall J, Pallis CA, Legg NJ. Clinical NMR imaging of the brain: 140 cases. AJR Am J Roentgenol 1982;139:215-36.

9. Carr DH, Brown J, Bydder GM, Weinmann JH, Speck U, Thomas DJ, Young IR. Intravenous chelated gadolinium as a contrast agent in NMR imaging of cerebral tumours. Lancet 1984;1:484-486.

10. Schörner W, Felix R, Laniado M, Lange L, Weinmann JH, Claussen C, Fiegler W, Speck U, Kazner E. Human testing of the nuclear spin tomographic contrast medium gadolinium-DPTA. Tolerance, contrast affect and the 1st clinical results. Rofo 1984;140:493-500.

11. Dixon WT. Simple proton spectroscopic imaging. Radiology 1984;153:189-94.

12. Le Bihan D, Breton E, Lallemand D, Grenier P, Cabanis E, Laval-Jeantet M. MR imaging of intravoxel incoherent motioins: application to diffusion and perfusion in neurologic disorders. Radiology 1986;161:401-7.

13. Moseley ME, Cohen Y, Kucharczyk J, Mintorovitch J, Asgari HS, Wendland MF, Tsuruda J, Norman D. Diffusion-weighted MR imaging of anisotropic water diffusion in cat central nervous system. Radiology 1990;176:439-45.

14. Moseley ME, Cohen Y, Mintorovitch J, Chileuitt L, Shimizu H, Kucharczyk J, Wendland MF, Weinstein PR. Early detection of regional cerebral ischemia in cats: comparison of diffusion- and T2-weighted MRI and Spectroscopy. Magn Reson Med 1990;14:330-46.

15. Hajnal JV, Doran M, Hall AS, Collins AG, Oatridge A, Pennock JM, Young IR, Bydder GM. MR imaging of anisotropically restricted diffusion of water in the nervous system: technical, anatomic, and pathologic considerations. J Comput Assist Tomogr 1991;15:1-18.

16. Takahara T, Imai Y, Yamashita T, Yasuda S, Nasu S, Van Cauteren M. Diffusion weighted whole body imaging with 
background body signal suppression (DWIBS): technical improvement using free breathing, STIR and high resolution 3D display. Radiat Med 2004;22:275-82.

17. Young IR, Khenia S, Thomas DG, Davis CH, Gadian DG Cox IJ, Ross BD, Bydder GM. Clinical magnetic susceptibility mapping of the brain. J Comput Assist Tomogr 1987;11:2-6.

18. Edelman RR, Johnson K, Buxton R, Shoukimas G, Rosen BR, Davis KR, Brady TJ. MR of hemorrhage: a new approach. AJNR Am J Neuroradiol 1986;7:751-6.

19. Bydder GM, Pennock JM, Porteous R, Dubowitz LM, Gadian DG, Young IR. MRI of intracerebral haematoma at low field $(0.15 \mathrm{~T})$ using $\mathrm{T} 2$ dependent partial saturation sequences. Neuroradiology 1988;30:367-71.

20. Haacke EM, Xu Y, Cheng YC, Reichenbach JR. Susceptibility weighted imaging (SWI). Magn Reson Med 2004;52:612-8.

21. Fretz CJ, Elizondo G, Weissleder R, Hahn PF, Stark DD, Ferrucci JT Jr. Superparamagnetic iron oxide-enhanced MR imaging: pulse sequence optimization for detection of liver cancer. Radiology 1989;172:393-7.

22. Ogawa S, Lee TM, Kay AR, Tonk DW. Brain magnetic resonance imaging with contrast dependent on blood oxygenation. Proc Natl Acad Sci U S A 1990;87:9868-72.

23. De Coene B, Hajnal JV, Gatehouse P, Longmore DB, White SJ, Oatridge A, Pennock JM, Young IR, Bydder GM. MR of the brain using fluid-attenuated inversion recovery (FLAIR) pulse sequences. AJNR Am J Neuroradiol 1992;13:1555-64.

24. Mathews VP, Caldemeyer KS, Lowe MJ, Greenspan SL, Weber DM, Ulmer JL. Brain: Gadolinium-enhanced fast fluid-attenuated inversion recovery MR imaging. Radiology 1999;211:257-63.

25. Balaban RS, Ceckler TL. Magnetization transfer contrast in magnetic resonance imaging. Magn Reson Q 1992;8:116-37.

26. Muller RN, Marsh MJ, Bernardo ML, Lauterbur PC. True 3-D imaging of limbs by NMR zeugmatography with off-resonance irradiation. Eur J Radiol 1983;3:286-90.

27. Hajnal JV, Baudouin CJ, Oatridge A, Young IR, Bydder GM. Design and implementation of magnetization transfer pulse sequences for clinical use. J Comput Assist Tomogr 1992;16:7-18.

28. Bergin CJ, Pauly JM, Macovski A. Lung parenchyma: projection reconstruction MR imaging. Radiology 1991;179:777-81.

29. Robson MD, Gatehouse PD, Bydder M, Bydder GM. Magnetic resonance: an introduction to ultrashort TE
(UTE) imaging. J Comput Assist Tomogr 2003;27:825-46. 30. Lee J, Hyun JW, Lee J, Choi EJ, Shin HG, Min K, Nam Y, Kim HJ, Oh SH. So You Want to Image Myelin Using MRI: An Overview and Practical Guide for Myelin Water Imaging. J Magn Reson Imaging. 2020. [Epub ahead of print]. doi:10.1002/jmri.27059.

31. Laule C, Vavasour IM, Moore GRW, Oger J, Li DKB, Paty DW, MacKay AL. Water content and myelin water fraction in multiple sclerosis. J Neurol 2004;251:284-93.

32. Mugler JP, Brookeman JR. Three-dimensional magnetization-prepared rapid gradient-echo imaging (3D MP RAGE). Magn Reson Med 1990;15:152-7.

33. Marques JP, Kober T, Krueger G, van der Zwaag W, Van de Moortele P-F, Gruetter R. MP2RAGE, a self bias-field corrected sequence for improved segmentation and T1mapping at high field. NeuroImage 2010;49:1271-81.

34. Kecskemeti S, Samsonov A, Hurley SA, Dean DC, Field A, Alexander AL. MPnRAGE: a technique to simultaneously acquire hundreds of differently contrasted MP-RAGE images with applications to quantitative T1 mapping. Magn Reson Med 2016;75:1040-53.

35. Mozes FE, Tunnicliffe EM, Moolla A, Marjot T, Levick CK, Pavlides M, Robson MD. Mapping tissue water T1 in the liver using the MOLLI T1 method in the presence of fat, iron and B0 inhomogeneity. NMR Biomed 2019;32:e4030.

36. Kimura T, Ikedo M, Takemoto S. Hybrid of oppositecontrast MR angiography (HOP-MRA) combining timeof-flight and flow sensitive black-blood contrasts. Magn Reson Med 2009;62:450-8.

37. Kimura T, Ikedo M, Takemoto S. Phase enhancement for time-of-flight and flow-sensitive black-blood MR angiography. Magn Reson Med 2011;66:437-47.

38. Holstila M, Virtanen KA, Grönroos TJ, Laine J, Lepomäki V, Saunavaara J, Lisinen I, Komu M, Hannukainen JC, Nuutila P, Parkkola R, Borra RJH. Measurement of brown adipose tissue mass using a novel dual-echo magnetic resonance imaging approach: a validation study. Metabolism 2013;62:1189-98.

39. Madhuranthakam AJ, Lee KS, Yassin A, Brittain JH, Pedrosa I, Rofsky NM, Alsop DC. Improved short tau inversion recovery (iSTIR) for increased tumor conspicuity in the abdomen. MAGMA 2014;27:245-55.

40. Fan S, Ma Y, Lu X, Du J, Bydder GM, Szeverenyi NM. Demonstration of abnormal cortical layers in Alzheimer's disease using subtracted tissue attenuated inversion (STAIR) pulse sequences. Honolulu, USA: ISMRM, 2017:2362.

41. Speckter H, Bido J, Hernandez G, Rivera D, Suazo L, Valenzuela S, Fermin R, Oviedo J, Foerster B, Gonzalez 
C, Stoeter P. Inversion recovery sequences improve delineation of optic pathways in the proximity of suprasellar lesions. J Radiosurg SBRT 2018;5:115-22.

42. Anderson KW, Lasic S, Lundell H, Nilsson M, Topgaard D, Sellebjerg F, Szczepankiewicz F, Siebner HR, Blinkenberg M, Dyrby TB. Disentangling white-matter damage from physiological fiber orientation dispersion in multiple sclerosis, Brain Communications 2020. doi: 10.1093/braincomms/fcaa077.

43. Ma YJ, Searleman AC, Jang H, Wong J, Chang EY, CoreyBloom J, Bydder GM, Du J. Whole-brain myelin imaging using 3D Double-Echo Sliding Inversion Recovery Ultrashort Echo Time (DESIRE UTE) MRI. Radiology 2020;294:362-74.

44. Ma YJ, Jang H, Wei Z, Cai Z, Xue Y, Chang EY, Bydder GM, Corey-Bloom J, Du J. Myelin imaging in human brain using a Short TR Adiabatic Inversion Recovery Prepared Ultrashort Echo Time (STAIR-UTE) MRI sequence in Multiple Sclerosis. Radiology 2020. [in press].

45. Ma YJ, Jang H, Chang EY, Hiniker A, Head BP, Lee RR, Corey-Bloom J, Bydder GM, Du J. Ultrashort echo time (UTE) magnetic resonance imaging of myelin: technical developments and challenges. Quant Imaging Med Surg 2020;10:1186-203.

46. Carlin D, Orton MR, Collins D, deSouza NM. Probing structure of normal and malignant prostate tissue before and after radiation therapy with luminal water fraction and Diffusion-Weighted MRI. J Magn Reson Imaging 2019;50:619-27.

47. Hectors SJ, Said D, Gnerre J, Tewari A, Taouli B. Luminal Water Imaging: Comparison With Diffusion-Weighted Imaging (DWI) and PI-RADS for Characterization of Prostate Cancer Aggressiveness. J Magn Reson Imaging 2020;52:271-9.

48. Yu AC, Badve C, Ponsky LE, Pahwa S, Dastmalchian S, Rogers M, Jiang Y, Margevicius S, Schluchter M, Tabayoyong W, Abouassaly R, McGivney D, Griswold MA, Gulani V. Development of a combined MR fingerprinting and diffusion examination for prostate cancer. Radiology 2017;283:729-38.

49. Panda A, Obmann VC, Lo W-C, Margevicius S, Jiang Y, Schluchter M, Patel IJ, Nakamoto D, Badve C, Griswold MA, Jaeger I, Ponsky LE, Gulani V. MR fingerprinting and ADC mapping for characterization of lesions in the transition zone of the prostate gland. Radiology 2019;292:685-94.

50. Barrett T, Vargas HA, Akin O, Goldman DA, Hricak H. Value of the hemorrhage exclusion sign on T1-weighted prostate MR images for the detection of prostate cancer. Radiology 2012;263:751-7.

51. Gupta RT, Mehta KA, Turkbey B, Verma S. PI-RADS: Past, present, and future. J Magn Reson Imaging 2020;52:33-53.

52. Padhani AR, Schoots I, Villeirs G. Contrast Medium or No Contrast Medium for Prostate Cancer Diagnosis. That Is the Question. J Magn Reson Imaging 2020. doi:10.1002/ jmri.27180.

53. Westphalen AC, McCulloch CE, Anaokar JM, Arora S, Barashi NS, Barentsz JO, Bathala TK, Bittencourt LK, Booker MT, Braxton VG, Carroll PR, Casalino DD, Chang SD, Coakley FV, Dhatt R, Eberhardt SC, Foster BR, Froemming AT, Fütterer JJ, Ganeshan DM. Variability of the positive predictive value of PI-RADS for prostate MRI across 26 centers: experience of the Society of Abdominal Radiology prostate cancer disease-focused panel. Radiology 2020;296:76-84.

54. Du J, Takahashi AM, Bae WC, Chung CB, Bydder GM. Dual inversion recovery, ultrashort echo time (DIR UTE) imaging: creating high contrast for short-T(2) species. Magn Reson Med 2010;63:447-55.

55. Burstein D, Velyvis J, Scott KT, Stock KW, Kim Yj, Jaramillo D, Boutin RD, Gray ML. Protocol issues for delayed Gd(DTPA) (2-)-enhanced MRI (dGEMRIC) for clinical evaluation of articular cartilage. Magn Reson Med 2001;45:36-41.

56. Zhu A, Hernando D, Johnson KM, Reeder SB. Characterized short $\mathrm{T} 2$ * signal apparent in the liver using ultrashort TE chemical shift encoded MRI at $1.5 \mathrm{~T}$ and 3.0T. Magn Reson Med 2019;82:2032-45.

57. Wood JC, Enriquez C, Ghugre N, Tyzka JM, Carson S, Nelson MD, Coates TD. MRI R2 and R2* mapping accurately estimates hepatic iron concentration in transfusion-dependent thalassemia and sickle cell disease patients. Blood 2005;106:1460-5.

58. Messiou C, Hillengass J, Delorme S, Lecouvet ME, Moulopoulos LA, Collins DJ, Blackledge MD, Abilgaard N, Østergaard B, Schlemmer H-P, Landgren O, Asmussen JT, Kaiser MF, Padhani A. Guidelines for acquisition, interpretation, and reporting of whole-body MRI in myeloma: MYeloma Response Assessment and Diagnosis System (MY-RADS). Radiology 2019;291:5-13.

59. Padhani AR, Lecouvet FE, Tunariu N, Koh D-M, De Keyzer F, Collins DJ, Sala E, Schlemmer HP, Petralia G, Vargas HA, Fanti S, Tombal HB, de Bono J. MEtastatis Reporting and Data System for Prostate Cancer: Practical Guidelines for Acquisition, Interpretation, and Reporting 
of Whole-body Magnetic Resonance Imaging-based Evaluations of Multiorgan Involvement in Advanced Prostate Cancer. Eur Urol 2017;71:81-92.

60. Ma YJ, Chen Y, Li L, Cai Z, Wei Z, Jerban S, Jan H, Chang EY, Du J. Trabecular bone imaging using a 3D adiabatic inversion recovery prepared ultrashort TE Cones sequence at 3T. Magn Reson Med 2020;83:1640-51.

61. Kober T, Granziera C, Ribes D, Browaeys P, Schluep M, Meuli R, Frackowiak R, Gruetter R, Krueger G. MP2RAGE multiple sclerosis Magnetic Resonance Imaging at 3T. Invest Radiol 2012;47:346-52.

Cite this article as: Ma YJ, Shao H, Fan S, Lu X, Du J, Young IR, Bydder GM. New options for increasing the sensitivity, specificity and scope of synergistic contrast magnetic resonance imaging (scMRI) using Multiplied, Added, Subtracted and/or FiTted (MASTIR) pulse sequences. Quant Imaging Med Surg 2020;10(10):2030-2065. doi: 10.21037/qims-20-795 